\title{
Cognition and type 2 diabetes : the interplay of risk factors
}

Citation for published version (APA):

Spauwen, P. J. J. (2015). Cognition and type 2 diabetes : the interplay of risk factors. [Doctoral Thesis, Maastricht University]. Uitgeverij BOXPress. https://doi.org/10.26481/dis.20150923ps

Document status and date:

Published: 01/01/2015

DOI:

10.26481/dis.20150923ps

Document Version:

Publisher's PDF, also known as Version of record

\section{Please check the document version of this publication:}

- A submitted manuscript is the version of the article upon submission and before peer-review. There can be important differences between the submitted version and the official published version of record.

People interested in the research are advised to contact the author for the final version of the publication, or visit the DOI to the publisher's website.

- The final author version and the galley proof are versions of the publication after peer review.

- The final published version features the final layout of the paper including the volume, issue and page numbers.

Link to publication

\footnotetext{
General rights rights.

- You may freely distribute the URL identifying the publication in the public portal. please follow below link for the End User Agreement:

www.umlib.nl/taverne-license

Take down policy

If you believe that this document breaches copyright please contact us at:

repository@maastrichtuniversity.nl

providing details and we will investigate your claim.
}

Copyright and moral rights for the publications made accessible in the public portal are retained by the authors and/or other copyright owners and it is a condition of accessing publications that users recognise and abide by the legal requirements associated with these

- Users may download and print one copy of any publication from the public portal for the purpose of private study or research.

- You may not further distribute the material or use it for any profit-making activity or commercial gain

If the publication is distributed under the terms of Article $25 \mathrm{fa}$ of the Dutch Copyright Act, indicated by the "Taverne" license above, 


\section{COGNITION AND TYPE 2 DIABETES}

THE INTERPLAY OF RISK FACTORS 
(C) Peggy J.J. Spauwen, Maastricht 2015

No part of this book may be reproduced or transmitted in any form or by any means, without prior permission in writing by the author, or when appropriate, by the publishers of the publications.

Layout: Tiny Wouters

Cover picture: Wendy van Rijen

Cover design: Hilarius Design

Printed by: Proefschriftmaken.nl || Uitgeverij BOXPress

Published by: Uitgeverij BOXpress, 's-Hertogenbosch

ISBN: 978-94-6295-344-4 


\title{
COGNITION AND TYPE 2 DIABETES
}

\author{
THE INTERPLAY OF RISK FACTORS
}

\section{PROEFSCHRIFT}

ter verkrijging van de graad van doctor aan de Universiteit Maastricht, op gezag van de Rector Magnificus, Prof. dr. L.L.G. Soete, volgens het besluit van het College van Decanen,

in het openbaar te verdedigen op woensdag 23 september 2015 om 12:00 uur

$$
\text { door }
$$

\section{Peggy Josephina Johannes Spauwen}

Geboren op 3 december 1985 te Heerlen 


\section{Promotores}

Prof. dr. F.R.J. Verhey

Prof. dr. C.D.A. Stehouwer

\section{Copromotor}

Dr. M.P.J. van Boxtel

\section{Beoordelingscommissie}

Prof. dr. R.W.H.M. Ponds, voorzitter

Prof. dr. G.J. Biessels (Utrecht University)

Prof. dr. H. Bosma

Prof. dr. C.A.J.M. Gaillard (University of Groningen)

Dr. M.M.J. van Greevenbroek

The research described in this thesis was performed at the Department of Psychiatry and Neuropsychology, School for Mental Health and Neuroscience, Maastricht University, Alzheimer Center Limburg, Maastricht, the Netherlands; and the Laboratory of Epidemiology and Population Science, National Institute on Aging, National Institutes of Health, Bethesda, Maryland, USA

The research for Chapter 5 was performed in collaboration with the National Institute on Aging and funded by a fellowship from Internationale Stichting Alzheimer Onderzoek.

Printing of this thesis was kindly supported by Alzheimer Nederland, Internationale Stichting Alzheimer Onderzoek, and Lundbeck. 


\section{Contents}

Chapter 1 General introduction

(Published in part in The Lancet Diabetes \& Endocrinology, 2014)

Chapter 2 Effects of type 2 diabetes on 12-year cognitive change:

17

Results from the Maastricht Aging study

(Diabetes Care, 2013)

Chapter 3 Associations of advanced glycation end-products with

cognitive functions in individuals with and without type 2

diabetes: The Maastricht Study

(The Journal of Clinical Endocrinology and Metabolism, 2015)

Chapter 4 Both low and high 24-hour diastolic blood pressure are associated

55

with worse cognitive performance in type 2 diabetes:

The Maastricht Study

(Adapted version published in Diabetes Care, 2015)

Chapter 5 Associations of fat and muscle tissue with cognitive status in older

adults: The AGES-Reykjavik study

Chapter 6 Lower verbal intelligence is associated with diabetic complications and slower walking speed in individuals with type 2 diabetes:

The Maastricht Study

Chapter 7 General discussion

(Published in part in The Lancet Diabetes \& Endocrinology, 2014)

Summary

Nederlandse samenvatting

Publications

Knowledge valorisation

Acknowledgements/Dankwoord

Curriculum Vitae 



\section{Chapter 1}

GENERAL INTRODUCTION 
Chapter 1 


\section{Risk factors for cognitive decline}

From middle age onwards, some of our cognitive abilities gradually decline. These abilities include processing speed, reasoning, memory, and executive functions (i.e., higher-order functions that allow for planning, goal-directed behavior, and attention). ${ }^{1}$ A certain amount of cognitive decline is part of normal aging. Some people, however, will experience accelerated cognitive decline, which may be indicative of a pathological condition, such as dementia. Dementia is a syndrome that is characterized by deterioration in memory, thinking, behavior and the ability to perform everyday activities independently. Dementia is one of the main causes of care dependency and disability at older ages. ${ }^{2}$ Today the number of people living with dementia worldwide is estimated to be 44 million, a number that is expected to almost double by 2030 and more than triple by $2050 .^{2}$

The most common cause of dementia is Alzheimer's disease (AD), which is characterized by progressive decline in cognitive functions, more specifically memory loss. It is associated with the accumulation of neurofibrillary tangles and amyloid plaques in the brain. ${ }^{3}$ Although the etiology of most dementia disorders (including AD) has not been elucidated, evidence has accumulated that vascular risk factors contribute to the development of dementia. Unfortunately, no disease modifying treatment to prevent or significantly delay dementia is currently available. Therefore, it is crucial to focus on modifiable risk factors, i.e. factors that can be modified in order to potentially decrease the risk of dementia and cognitive impairment. One important modifiable risk factor, which becomes more common with advancing age, is type 2 diabetes. ${ }^{2,4}$ Type 2 diabetes is a metabolic disorder that is characterized by high blood sugar levels resulting from defects in insulin secretion, action, or both. Other vascular risk factors that often co-occur with diabetes and also have been associated with the development of dementia include hypertension and obesity. ${ }^{2,4}$

In order to increase our insight into the underlying mechanisms of cognitive decline and dementia, and to become able to delay or prevent the development of such age-related conditions, we need to understand more deeply the cognitive trajectories and profiles of individuals in whom these vascular risk factors are present. Therefore, it is necessary to examine if and to what extent these risk factors are associated with a reduction in cognitive performance, which may be indicative of a cognitive disorder at a later stage.

In the first part of this thesis we will study the associations of type 2 diabetes, its associated vascular risk factors, and body composition with cognitive functioning. 


\section{Type 2 diabetes and cognitive change over time ${ }^{a}$}

Evidence is accumulating that type 2 diabetes is associated with cognitive impairment and dementia. In individuals older than 60 years, $7-13 \%$ of all dementia cases are estimated to be attributable to diabetes ${ }^{5}$ and the number of cases of diabetesassociated dementia is expected to increase because of the diabetes pandemic in our ageing societies. However, the natural course of cognitive decline in type 2 diabetes is not well understood. To understand the effect of diabetes on the development of cognitive impairment and dementia it is therefore necessary to examine the effect of diabetes on cognitive change over time.

It has been suggested that clinically relevant cognitive decline related to type 2 diabetes does not develop until age-related neurodegenerative changes in the brain start to occur (i.e., after age 60 years). ${ }^{5}$ However, effects of diabetes on cognitive decline have also been found in middle-aged adults. ${ }^{6,7}$ Additionally, some studies have shown that diabetes is associated with greater cognitive decline, ${ }^{8,9}$ although others did not confirm this. ${ }^{10,11}$ Different follow-up durations may explain these results as it has been suggested that effects of diabetes on cognitive change over time develop slowly. ${ }^{12}$ Furthermore, in some studies ${ }^{6,13}$ the extent of cognitive decline in new-onset diabetes was not clearly different from that in individuals without diabetes, suggesting the existence of a threshold for diabetes duration in the association between diabetes and cognitive decline. It is important to know if diabetes already may have an effect on cognition early in the disease, as this may be a phase for intervention aimed at delaying or preventing further cognitive decline.

Therefore the first research question that was addressed by this thesis is:

[1] What are the effects of prevalent and incident type 2 diabetes on cognitive change over time?

\section{Diabetes-associated vascular risk factors, body composition, and cognitive functioning}

Several mechanisms have been proposed to explain the association between diabetes and cognitive impairment. One mechanism includes the formation of advanced glycation end-products (AGEs), which are thought to be involved in the development of AD. ${ }^{14}$ This mechanism is particularly interesting, because AGEs can be readily measured in plasma and the skin.

AGEs are the result of the binding of proteins with glucose and their accumulation increases as a function of time and glucose concentration. ${ }^{15}$ Accumulation of AGEs is therefore increased in individuals with diabetes. ${ }^{15}$

\footnotetext{
a Published in part in Spauwen PJ and Stehouwer CD. Cognitive decline in type 2 diabetes. Lancet Diabetes Endocrinol. 2014;2:188-9.
} 
Interestingly, AGE accumulation in the brain has been linked to AD pathology. ${ }^{14,16}$ To date, only a few studies have tested associations of AGEs with cognitive functions and have shown associations of AGEs with cognitive impairment and decline, even in individuals without diabetes. ${ }^{17-19}$ Therefore, examination of the association between AGEs with multiple cognitive functions may provide a marker for cognitive impairment and might increase our knowledge about the etiology of cognitive decline and dementia.

Furthermore, diabetes is often accompanied by other well known vascular risk factors, which may affect the brain, including hypertension. As both diabetes and hypertension seem to affect cognitive functions independently, individuals with both risk factors may have an especially high risk of cognitive decline. This has indeed been shown by some ${ }^{20,21}$ but not all studies ${ }^{22,23}$ on this topic to date. The most valid way to assess blood pressure under natural conditions is by means of 24-hour ambulatory blood pressure measurement (ABPM). In addition, this assessment can be used to measure the natural difference in average daytime and nighttime blood pressure levels (nocturnal 'dipping'). Absence of nocturnal dipping in average blood pressure has been associated with cognitive impairment in previous studies. ${ }^{24,25}$ The study of associations between ambulatory blood pressure on the one hand and cognitive functions on the other in individuals with and without diabetes may therefore provide valuable information about which individuals are at an increased risk to develop cognitive impairment.

Midlife obesity, indicated by a high body mass index (BMI), is a risk factor for diabetes and has been associated with cognitive functioning and dementia. ${ }^{26,27}$ However, in late life, a lower BMI has been associated with a higher risk of dementia. ${ }^{28,29}$ Loss of body tissue, including fat and muscle tissue, may be a consequence of dementia, but could also have some negative effects on the brain. For example, while visceral fat (abdominal fat that surrounds the inner organs) has been shown to be metabolically active and to be strongly associated with insulin resistance, ${ }^{30}$ thigh subcutaneous fat (fat stored beneath the skin) has been associated with lower glucose and lipid levels. ${ }^{31}$ Losing visceral fat when entering old age may therefore be less detrimental or even beneficial compared with losing thigh subcutaneous fat. Therefore, a better insight into specific patterns of body composition associated with different levels of cognitive function may help us to better understand the underlying mechanisms associated with weight loss and cognitive impairment.

The following research questions were addressed regarding diabetes-associated vascular risk factors, body composition and cognitive functioning:

[2] Are higher levels of AGEs associated with worse cognitive performance? 
[3] What is the association of 24-hour blood pressure and nocturnal dipping status with cognitive functions in individuals with and without type 2 diabetes?

[4] What is the association of the amount of fat and muscle tissue with mild cognitive impairment and dementia in late life?

\section{Health literacy and diabetic complications}

Type 2 diabetes is a complex disease, which is not only associated with dementia, but also with several micro- and macrovascular complications, such as retinopathy, nephropathy, neuropathy, and cardiovascular disease. Important determinants of these complications, which are also important targets in diabetes management, include glycemic control, blood pressure, and LDL-cholesterol control. In order to achieve proper control of these parameters and to prevent development of complications, diabetic patients have to engage in self-care behaviors. Self-care behaviors in individuals with diabetes include: healthy eating, being physically active, self-monitoring of blood glucose, taking medication, problem solving, healthy coping, and risk reduction behaviors. ${ }^{32}$

To engage in these self-care behaviors, diabetic patients need to have a basic understanding of their disease and of the oral and written health instructions given by their health care providers. This ability is often referred to as health literacy, i.e. the degree to which individuals have the capacity to obtain, process, and understand basic health information and services needed to make appropriate health decisions. ${ }^{33}$

Inadequate health literacy appears to be quite common in diabetic patients, ${ }^{34}$ with numbers ranging from $10-44 \%$ depending on the study sample, ${ }^{34-37}$ and could be associated with glycemic control and diabetic complications. Indeed, some studies have found such adverse associations. ${ }^{35,38}$ In order to increase the chance of successful treatment and to decrease the risk of complications, it is important to identify patients who are at increased risk for developing complications. Verbal intelligence, as assessed with vocabulary tests, has been suggested to be a core constituent and a strong determinant of health literacy ${ }^{39}$ and could therefore be used to identify patients with an increased risk for diabetes-related end-organ damage.

In the last part of this thesis the following research question was addressed:

[5] Is a lower verbal intelligence associated with the presence of diabetic complications? 


\section{Datasets used in this thesis}

Datasets from three population-based studies were used in this thesis. Each of these datasets has its own focus and specialization. The Maastricht Aging Study (MAAS) was specifically designed to identify the determinants of successful cognitive aging, The Maastricht Study is focused on determinants and outcomes of cardiovascular risk factors, particularly type 2 diabetes, and The Age Gene/Environment Susceptibility (AGES)-Reykjavik Study was conceived and designed to study risk factors and genetic contributions to conditions of old age.

\section{The Maastricht Aging Study}

MAAS is a longitudinal cohort study on the determinants of cognitive aging. ${ }^{40}$ At baseline, 1,823 randomly selected participants underwent a comprehensive assessment of medical status, lifestyle, and anthropomorphic and cognitive functioning measures. The baseline assessment took place from 1993-1996 and was repeated 6 and 12 years after baseline. This dataset made it possible the study effects of baseline and incident diabetes on cognitive change over a long time.

\section{The Maastricht Study}

The Maastricht Study is an ongoing observational prospective population-based cohort study, which focuses on the etiology, pathophysiology, complications and comorbidities of type 2 diabetes mellitus and is characterized by an extensive phenotyping approach. ${ }^{41}$ This study includes multiple elaborate measurements of vascular risk factors, diabetic complications, and assessment of most important cognitive domains and verbal intelligence. The first dataset of The Maastricht Study included 866 participants, of whom 253 had type 2 diabetes, and was used to examine associations of diabetes and its associated vascular risk factors with cognitive performance and to examine associations of verbal intelligence with diabetic complications.

\section{AGES-Reykjavik Study}

The AGES-Reykjavik Study is a population-based cohort study, which consists of more than 5,000 older individuals living in Iceland. The aim of this study was to investigate risk factors, genetic susceptibility, and gene-environment interactions in relation to disease and disability in old age, as well as healthy aging. ${ }^{42}$ This dataset was used to examine associations of body composition with cognitive status in late life as it allowed us to use computed tomography (CT) scan data in a large sample of older individuals. 


\section{Outline of thesis}

To summarize, this thesis describes the role of type 2 diabetes, its associated vascular risk factors, and body composition with cognitive functions. In chapter 2 to 5 we examine if type 2 diabetes, its associated vascular risk factors, and body composition are predictors for cognitive functioning, while in the final chapter we examine if verbal intelligence may be used as a predictor of diabetic complications. More specifically:

In Chapter 2, the effects of baseline and incident type 2 diabetes on cognitive change over time are examined in participants of MAAS.

In Chapter 3, the associations of skin autofluorescence, a non-invasive marker for tissue AGEs, and plasma AGEs with performance on multiple cognitive tests are tested in participants of The Maastricht Study.

Chapter 4 examines linear and quadratic associations of 24-hour blood pressure with several cognitive functions stratified by diabetes status in The Maastricht Study. In addition, associations of dipping status with cognitive functions are examined in this chapter.

In Chapter 5, associations between several body composition measures, i.e. visceral fat, abdominal and thigh subcutaneous fat, and muscle tissue, and mild cognitive impairment $(\mathrm{MCl})$ and dementia are examined in older individuals who participated in the AGES-Reykjavik study.

In Chapter 6, data of individuals with type 2 diabetes participating in The Maastricht Study are used to examine the association of verbal intelligence with diabetic complications and their most important determinants, and with walking speed, a measure of physical functioning and strong predictor of overall health status. In addition, in this chapter we examine the extent to which these associations can be explained by educational level.

In Chapter 7, all results that are presented in the earlier chapters are placed in a wider context, together with some reflection on methodological issues and the clinical and scientific implications of our studies. 


\section{References}

1. Deary IJ, Corley J, Gow AJ, et al. Age-associated cognitive decline. Br Med Bull 2009;92:135-52

2. Prince M, Albanese E, Guerchet M, Prina M. World Alzheimer report 2014. Dementia and risk reduction: an analysis of protective and modifiable risk factors. London, Alzheimer's Disease International, 2014

3. LaFerla FM, Oddo S. Alzheimer's disease: Abeta, tau and synaptic dysfunction. Trends Mol Med 2005;11:170-6

4. Barnes DE, Yaffe K. The projected effect of risk factor reduction on Alzheimer's disease prevalence. Lancet Neurol 2011;10:819-28

5. Biessels GJ, Deary IJ, Ryan CM. Cognition and diabetes: a lifespan perspective. Lancet Neurol 2008;7:184-90

6. Nooyens AC, Baan CA, Spijkerman AM, Verschuren WM. Type 2 diabetes and cognitive decline in middle-aged men and women: the Doetinchem Cohort Study. Diabetes Care 2010;33:1964-9

7. Tuligenga RH, Dugravot A, Tabak AG, et al. Midlife type 2 diabetes and poor glycaemic control as risk factors for cognitive decline in early old age: a post-hoc analysis of the Whitehall II cohort study. Lancet Diabetes Endocrinol 2014;2:228-35

8. Yaffe K, Blackwell T, Kanaya AM, Davidowitz N, Barrett-Connor E, Krueger K. Diabetes, impaired fasting glucose, and development of cognitive impairment in older women. Neurology 2004;63: 658-63

9. Wessels AM, Lane KA, Gao S, Hall KS, Unverzagt FW, Hendrie HC. Diabetes and cognitive decline in elderly African Americans: a 15-year follow-up study. Alzheimers Dement 2011;7:418-24

10. Fischer AL, de Frias CM, Yeung SE, Dixon RA. Short-term longitudinal trends in cognitive performance in older adults with type 2 diabetes. J Clin Exp Neuropsychol 2009;31:809-22

11. van den Berg E, Reijmer YD, de Bresser J, Kessels RP, Kappelle LJ, Biessels GJ. A 4 year follow-up study of cognitive functioning in patients with type 2 diabetes mellitus. Diabetologia;53:58-65

12. Reijmer YD, van den Berg E, Ruis C, Kappelle LJ, Biessels GJ. Cognitive dysfunction in patients with type 2 diabetes. Diabetes Metab Res Rev 2010;26:507-19

13. Yaffe K, Falvey C, Hamilton N, et al. Diabetes, glucose control, and 9-year cognitive decline among older adults without dementia. Arch Neurol 2012;69:1170-5

14. Li J, Liu D, Sun L, Lu Y, Zhang Z. Advanced glycation end products and neurodegenerative diseases: mechanisms and perspective. J Neurol Sci 2012;317:1-5

15. Brownlee M. Advanced protein glycosylation in diabetes and aging. Annu Rev Med 1995;46:223-34

16. Valente T, Gella A, Fernandez-Busquets X, Unzeta M, Durany N. Immunohistochemical analysis of human brain suggests pathological synergism of Alzheimer's disease and diabetes mellitus. Neurobiol Dis 2010;37:67-76

17. Chen G, Cai L, Chen B, et al. Serum level of endogenous secretory receptor for advanced glycation end products and other factors in type 2 diabetic patients with mild cognitive impairment. Diabetes care 2011;34:2586-90

18. Yaffe K, Lindquist $\mathrm{K}$, Schwartz $\mathrm{AV}$, et al. Advanced glycation end product level, diabetes, and accelerated cognitive aging. Neurology 2011;77:1351-6

19. Moran C, Munch G, Forbes JM, et al. Type 2 diabetes mellitus, skin autofluorescence and brain atrophy. Diabetes 2015;64:279-83

20. Hassing LB, Hofer SM, Nilsson SE, et al. Comorbid type 2 diabetes mellitus and hypertension exacerbates cognitive decline: evidence from a longitudinal study. Age Ageing 2004;33:355-61

21. Xu WL, Qiu CX, Wahlin A, Winblad B, Fratiglioni L. Diabetes mellitus and risk of dementia in the Kungsholmen project: a 6-year follow-up study. Neurology 2004;63:1181-6

22. Gregg EW, Yaffe K, Cauley JA, et al. Is diabetes associated with cognitive impairment and cognitive decline among older women? Study of Osteoporotic Fractures Research Group. Arch Intern Med 2000;160:174-80

23. Korf ES, van Straaten EC, de Leeuw FE, et al. Diabetes mellitus, hypertension and medial temporal lobe atrophy: the LADIS study. Diabet Med 2007;24:166-71

24. van Boxtel MP, Gaillard C, Houx PJ, Buntinx F, de Leeuw PW, Jolles J. Is nondipping in $24 \mathrm{~h}$ ambulatory blood pressure related to cognitive dysfunction? J Hypertens 1998;16:1425-32 
25. Okuno J, Yanagi H. Cognitive impairment and nocturnal blood pressure fall in treated elderly hypertensives. Environ Health Prev Med 2003;8:124-32

26. Cournot M, Marquie JC, Ansiau D, et al. Relation between body mass index and cognitive function in healthy middle-aged men and women. Neurology 2006;67:1208-14

27. Luchsinger JA, Gustafson DR. Adiposity, type 2 diabetes, and Alzheimer's disease. J Alzheimers Dis 2009;16:693-704

28. Power BD, Alfonso H, Flicker L, Hankey GJ, Yeap BB, Almeida OP. Body adiposity in later life and the incidence of dementia: the health in men study. PLoS One 2011;6:e17902

29. Fitzpatrick AL, Kuller $\mathrm{LH}$, Lopez $\mathrm{OL}$, et al. Midlife and late-life obesity and the risk of dementia: cardiovascular health study. Arch Neurol 2009;66:336-42

30. Carr DB, Utzschneider KM, Hull RL, et al. Intra-abdominal fat is a major determinant of the National Cholesterol Education Program Adult Treatment Panel III criteria for the metabolic syndrome. Diabetes 2004;53:2087-94

31. Manolopoulos KN, Karpe F, Frayn KN. Gluteofemoral body fat as a determinant of metabolic health. Int J Obes (Lond) 2010;34:949-59

32. American Association of Diabetes Educators. AADE7 Self-Care Behaviors. Diabetes Educ 2008;34: 445-9

33. Institute of Medicine. Health literacy: A Prescription to End Confusion. Washington DC, The National Academies, 2004

34. Williams MV, Baker DW, Parker RM, Nurss JR. Relationship of functional health literacy to patients' knowledge of their chronic disease. A study of patients with hypertension and diabetes. Arch Intern Med 1998;158:166-72

35. Schillinger D, Grumbach K, Piette J, et al. Association of health literacy with diabetes outcomes. JAMA 2002;288:475-82

36. Morris NS, MacLean CD, Littenberg B. Literacy and health outcomes: a cross-sectional study in 1002 adults with diabetes. BMC Fam Pract 2006;7:49

37. Mancuso JM. Impact of health literacy and patient trust on glycemic control in an urban USA population. Nurs Health Sci 2010;12:94-104

38. Powell CK, Hill EG, Clancy DE. The relationship between health literacy and diabetes knowledge and readiness to take health actions. Diabetes Educ 2007;33:144-51

39. Deary IJ, Gale CR, Stewart MC, et al. Intelligence and persisting with medication for two years: Analysis in a randomised controlled trial. Intelligence 2009;37:607-12

40. Jolles J, Houx PJ, van Boxtel MP, Ponds RW, (Eds.). Maastricht Aging Study: Determinants of cognitive aging. Maastricht, Neuropsych Publishers, 1995

41. Schram MT, Sep SJ, van der Kallen CJ, et al. The Maastricht Study: an extensive phenotyping study on determinants of type 2 diabetes, its complications and its comorbidities. Eur J Epidemiol 2014;29: 439-51

42. Harris TB, Launer L, Eiriksdottir G, et al. Age, Gene/Environment Susceptibility-Reykjavik Study: multidisciplinary applied phenomics. Am J Epidemiol 2007;165:1076-87 


\section{Chapter 2}

EFFECTS OF TYPE 2 DIABETES ON 12-YEAR COGNITIVE CHANGE: RESULTS FROM THE MAASTRICHT AGING STUDY

PJJ Spauwen, S Köhler, FRJ Verhey, CDA Stehouwer, MPJ van Boxtel Diabetes Care 2013;36:1554-61 


\section{Abstract}

\section{Objective}

To examine the effects of baseline and incident diabetes on change in cognitive function over 12 years.

\section{Research design and methods}

A sample of 1,290 individuals aged 40 years and older at baseline, participating in the Maastricht Aging Study (MAAS), were cognitively tested at baseline, after six years, and after 12 years. Of these, 68 participants had type 2 diabetes at baseline, and 54 and 57 had incident diabetes at 6-year and 12-year follow-up, respectively. Changes in performance on tests of information processing speed, executive function, and verbal memory from baseline to 6 -year and to 12-year follow-up were compared between groups using linear mixed models. Effects of diabetes on cognitive decline were adjusted for demographic variables, history of smoking, alcohol intake, and comorbid conditions, including hypertension, cardiovascular disease, BMI, and depression.

\section{Results}

Participants with baseline diabetes showed larger decline in information processing speed (estimate, $-7.64, P<0.01$ ), in executive function $(21.82, P<0.01)$, and in delayed word recall $(-1.35, P<0.05)$ over 12-year follow-up compared with controls. No significant difference in decline was observed for immediate word recall. Compared with controls, participants with incident diabetes showed subtle early decline in information processing speed only. Interestingly, they did not show larger decline in any other cognitive domain.

\section{Conclusions}

Individuals with baseline type 2 diabetes show accelerated cognitive decline, particularly in information processing speed and executive function, compared with individuals without diabetes. In incident diabetes, decline in speed becomes detectable first and cognitive decline seems to increase with increasing exposure time. 


\section{Introduction}

Type 2 diabetes is associated with an increased risk of cognitive impairment and dementia. ${ }^{1-3}$ Mechanisms underlying these associations are not well understood, but macro- and microvascular disease might play an important role given their prevalence in diabetes, ${ }^{1}$ and dementia. ${ }^{4}$ Despite the increased risk of dementia and cognitive impairment, studies into the cognitive trajectories of patients with type 2 diabetes have been inconclusive.

Although some studies showed that cognitive decline in diabetic patients is largely within the range of normal aging, ${ }^{5-7}$ others have shown a greater cognitive decline in diabetic patients compared with controls. ${ }^{8-10}$ However, in most studies the observed differences were generally small and the follow-up duration was relatively short, with an average of five years. ${ }^{11}$ It might be that diabetes-associated cognitive dysfunction takes several years to emerge and that the follow-up durations of most previous studies are too short to detect substantial differences in cognitive decline. In addition, studies focused on baseline diabetes and hence the effect of incident diabetes on cognitive change over time is not adequately addressed. It is important to study this effect to increase the understanding of cognitive changes early in the disease.

Therefore, this study presents 12-year follow-up data from a population-based prospective cohort study, to investigate the effects of baseline and incident type 2 diabetes on decline in several cognitive domains. We hypothesized that a) participants with baseline type 2 diabetes would show a larger cognitive decline than participants without diabetes, b) given their shorter exposure time, the rate of decline of those with incident diabetes at follow-up would lie between that of participants with baseline diabetes and those without diabetes, and c) associations between diabetes and cognitive decline could not be fully explained by risk factors that are potentially related to both diabetes and cognitive function.

\section{Methods}

\section{Sample}

The present study was performed as part of the Maastricht Aging Study (MAAS), a longitudinal study of determinants of cognitive aging. ${ }^{12}$ A total of 10,396 people were sampled from the RNH (Registration Network Family Practices), a patient register of collaborating general practitioners in the province of Limburg the Netherlands. ${ }^{13}$ Patients in the RNH register are considered representative of the Limburg and Dutch population with respect to demographic characteristics (age, gender, and educational level). ${ }^{12}$ Of these 10,396 people, 4,490 (43.2\%) were willing to participate, 3,531 (34\%) refused participation, and $2,375(22.8 \%)$ did not respond to the written request. 
Medical exclusion criteria for baseline assessment were defined as active or inactive medical conditions in the RNH problem list that could interfere with normal cognitive function, including coma (only active), cerebrovascular pathology, a tumor of the nervous system, congenital malformation of the nervous system, multiple sclerosis, Parkinsonism, epilepsy (all types), dementia, organic psychosis, schizophrenia, affective psychosis, and mental retardation. Before participation in MAAS, all 4,490 participants were screened by telephone for the following medical conditions not documented in the RNH database: history of transient ischemic attacks, brain surgery, hemodialysis for renal failure, electroconvulsive therapy, and regular use of psychotropic drugs. Based on this interview, 301 participants were excluded. Of the remaining 4,189 participants, 1,823 (43.5\%) were randomly selected, stratified for age (12 discrete age groups from 24 to 81 years), sex, and level of general ability (low/high), and were equally distributed over four demographically identical test panels. The local ethics committee approved the study, and all participants gave informed consent. At baseline, these 1,823 participants underwent a comprehensive assessment of medical status, lifestyle, and anthropomorphic and cognitive functioning measures. The baseline assessment took place from 1993-1996 and was repeated six and 12 years after baseline. Only participants aged 40 years or older were included in the present study in order to make the sample more homogeneous. In addition, we excluded 4 participants with type 1 diabetes. The final study sample therefore consisted of 1,290 participants.

\section{Diabetes status}

Presence of type 2 diabetes was based on a diagnosis made by a physician (as reported by the study participant) and current medication use for diabetes reported in a questionnaire. Diagnosis of type 2 diabetes based on self-report has previously been used in large cohort studies ${ }^{10,14}$ and has been shown to be a reliable estimate of the actual prevalence of diabetes. ${ }^{15}$ To make a distinction between type 1 and type 2 diabetes, participants were considered to have diabetes type 2 when they used oral antidiabetic drugs, started using insulin at or after the age of 40 , or were diagnosed with diabetes at or after the age of 40 . Diabetes status was assessed at baseline, at 6-year, and at 12-year follow-up.

\section{Covariates}

Several potentially health-related conditions that might influence the association between type 2 diabetes and cognitive change over time were taken into account. These included self-report of present or past history of smoking (yes/no), alcohol intake (according to WHO guidelines less/more than 21 standard consumption per week for females, less/ more than 35 consumptions per week for males), ${ }^{16}$ a history of cardiovascular disease (yes/no) as reported in a questionnaire, and BMI (weigh in $\mathrm{kg} /$ length in $\mathrm{m}^{2}$ ). Blood pressure was measured three times at five minutes interval on 
the left arm using an automatic recording device (Critikon Dinamap 8100; Critikon, Tampa, FL). Hypertension (yes/no) was defined as either a mean systolic blood pressure of $140 \mathrm{mmHg}$ or higher, a mean diastolic blood pressure of $90 \mathrm{mmHg}$ or higher, ${ }^{17}$ or current use of antihypertensive medication as reported in a questionnaire. Depressive symptoms were assessed with the 16-item depression subscale of the revised Symptom Checklist-90 (score range 16-80). ${ }^{18}$ Apolipoprotein (APOE) genotyping was determined on genomic DNA extracted from EDTA-anticoagulated blood using a polymerase chain reaction technique. ${ }^{19}$ The presence (yes/no) of the APOE \&4 (APOE-e4) risk allele, which is associated with an increased risk of dementia, ${ }^{20}$ was used in the present study. Furthermore, age, sex, and educational level (ranging from primary education (1) to university degree (8)) ${ }^{21}$ were included as demographic covariates. Three groups were made for educational level: low (level 1 to 2), middle (level 3 to 5), and high (level 6 to 8). Hypertension, a history of cardiovascular disease, BMI, and depression were measured at baseline and each follow-up assessment.

\section{Assessment of cognitive function}

Cognitive function was measured at baseline, at 6-year, and at 12-year follow-up with a battery of neuropsychological tests administered by psychologists and trained test assistants. An a priori selection of these neuropsychological tests was used to assess cognitive function in the current study. The Visual Verbal Learning Test (VVLT) ${ }^{22}$ was used to assess verbal memory. In this test, 15 non-related monosyllabic words are presented in five subsequent trials on a computer screen, followed by a recall phase immediately after each trial and a delayed recall phase 20 minutes after the test. The total number of correctly reproduced words in five trials was measured (immediate word recall), together with the number of correctly reproduced words 20 minutes after the last trial (delayed word recall). The Concept Shifting Test (CST) ${ }^{23}$ was used to measure executive function. In three trials the participants have to cross out as fast as possible 16 digits in ascending order (CST Part A), 16 letters in alphabetical order (CST Part B), and finally 8 digits and 8 letters in alternating order (CST Part C). The shifting score is calculated by subtracting the average time needed to finish part $A$ and $B$ from the time needed to finish part C. The Letter Digit Substitution Test (LDST) ${ }^{24}$ was used to assess information processing speed. Participants were instructed to match digits to letters, according to a key of letter/digit combinations at the top of the sheet, as quickly as possible within 90 seconds. The Mini-Mental State Examination (MMSE) ${ }^{25}$ broadly assesses several domains of cognitive functioning and was used to measure global cognitive functioning.

\section{Statistical analysis}

Differences between group characteristics at baseline were tested using independent sample t-tests for quantitative variables (age, BMI, depression score, MMSE score, 
baseline scores on cognitive tests), $\chi^{2}$-tests for qualitative variables (sex, history of cardiovascular disease, hypertension, history of smoking, alcohol intake, and APOE-e4 allele) and the Mann-Whitney U-test for the ordinal variable educational level. Linear regression was used to estimate the effect of baseline type 2 diabetes on cognitive performance at baseline. Next, separate linear mixed models (random effects models) were run to measure the crude and adjusted effect of baseline diabetes on change in cognition from baseline to 6-year (F1) and to 12-year follow-up (F2). An advantage of mixed models over analysis of variance with repeated measures is that it models timetrajectories without restricting analyses to study completers. The model included terms for a random intercept and a random slope. An unstructured covariance structure provided the best model fit. Terms for the fixed effects included the intercept, baseline diabetes, age and age ${ }^{2}$ (in order to control for nonlinear trends), sex, educational level, and baseline history of smoking, baseline alcohol intake, hypertension, history of cardiovascular disease, BMI, depression score, type 2 diabetes, time (F1, F2), as well as the interaction terms between F1 and diabetes and F2 and diabetes. APOE-e4 allele status (risk allele present/absent) was included as a covariate in an additional analysis as this was only measured in a subsample.

A second set of linear mixed models tested the crude and adjusted effect of incident type 2 diabetes on change in cognitive performance over time, excluding participants with baseline diabetes. This yielded three groups for comparison ( $1=$ no diabetes (reference), 2=incident diabetes at F1, 3=incident diabetes at F2). Hypertension, past or present cardiovascular disease, BMI, and depression score were now treated as time-dependent covariates, thereby allowing them to co-vary with diabetes status over time. To test whether loss to follow-up could be predicted by the observed variables, a logistic regression with lost-to-follow-up (yes/no) as dependent variable was performed. A $P$-value $<0.05$ (two-sided) was considered statistically significant. All analyses were done in IBM SPSS Statistics 19 (SPSS, Chicago, IL).

\section{Results}

At baseline, 1,290 participants (aged 40.0-82.7) were tested of whom 68 (5.3\%) had diabetes. At F1, 925 (75.7\%) participants without type 2 diabetes at baseline and 41 (60.3\%) participants with diabetes at baseline were still in the study. At F2, the sample sizes were $761(62.3 \%)$ and $21(30.9 \%)$ for the two groups respectively. The mean follow-up time was 8.7 years. Of the 1,222 participants without type 2 diabetes at baseline, 54 (4.4\%) participants developed type 2 diabetes between baseline and F1 and 57 (4.7\%) participants developed type 2 diabetes between F1 and F2. Having type 2 diabetes at baseline, being older, having a history of smoking, cardiovascular disease, higher BMI, or lower baseline MMSE score increased the risk of study dropout. 


\section{Baseline differences}

Baseline characteristics stratified by diabetes status are shown in Table 2.1. Compared with participants without diabetes, participants with diabetes were older, had a lower educational level, had a higher BMI, were more likely to have hypertension and a history of cardiovascular disease, and performed worse on all cognitive measures. These between group differences in cognitive performance were still significant after adjustment for demographic variables, history of smoking, alcohol use and comorbid conditions. There were no statistically significant differences in sex, alcohol intake, history of smoking, presence of APOE-e4 allele and depression score between groups. Participants with incident diabetes at F1 were more likely to be male, were significantly older, had a higher BMI, were more likely to have a history of cardiovascular disease and hypertension, and had a lower depression score than participants who were free of diabetes during the whole follow-up period. Participants with incident diabetes at F2 were more likely to have hypertension and had a significantly higher BMI than participants without diabetes during the whole follow-up period. At baseline, i.e. before a diagnosis of diabetes was made, there were no significant differences in measures for information processing speed $(P=0.24)$, concept shifting $(P=0.28)$, immediate word recall $(P=0.08)$ and delayed word recall $(P=0.06)$ between participants with incident diabetes at F1 and controls. Likewise, there were no significant differences in information processing speed $(P=0.57)$, concept shifting $(P=0.35)$, immediate word recall $(P=0.26)$ and delayed word recall $(P=0.06)$ between participants with incident diabetes at $F 2$ and controls. However, participants with incident diabetes at F2 had a significantly higher MMSE score than participants without diabetes.

\section{Baseline diabetes and 12-year cognitive decline}

Results of the association between baseline diabetes and cognitive change over time are presented in Table 2.2. In crude analyses (model 1), participants with diabetes showed a significantly larger decline in information processing speed and concept shifting from baseline to F1 and to F2 compared with those without diabetes. 


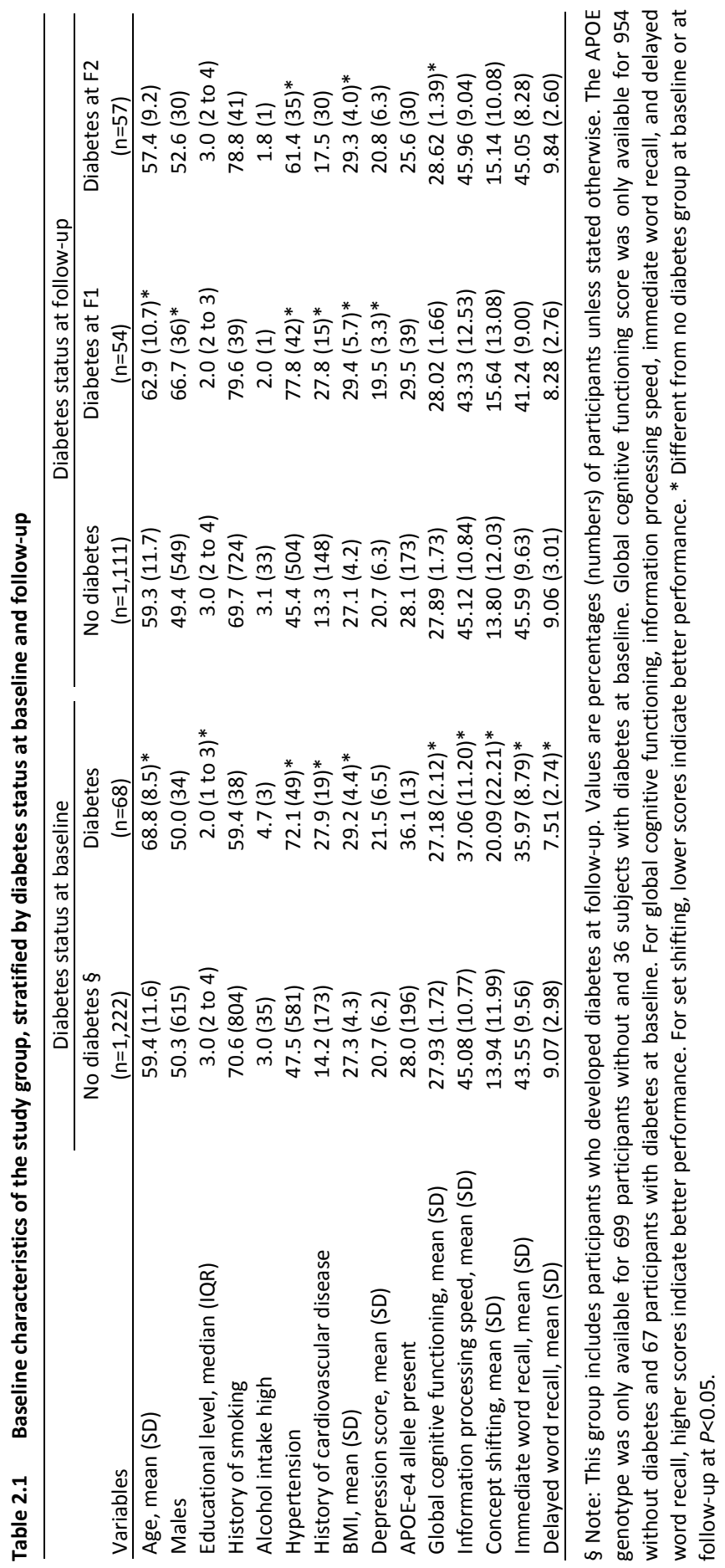


Table 2.2 Change in cognitive performance (95\% confidence interval) from baseline to 6-year followup (F1) and to 12 year follow-up (F2) in participants with baseline diabetes relative to participants without diabetes before and after adjustment for various covariates

\begin{tabular}{|c|c|c|c|}
\hline & & From baseline to F1 & From baseline to $\mathrm{F} 2$ \\
\hline \multirow[t]{3}{*}{ Information processing speed } & Model 1 & $-4.57(-6.39 \text { to }-2.74)^{* *}$ & $-7.99(-10.72 \text { to }-5.26)^{* *}$ \\
\hline & Model 2 & $-4.51(-6.36 \text { to }-2.66)^{* *}$ & $-7.74(-10.52 \text { to }-4.98)^{* *}$ \\
\hline & Model 3 & $-4.82(-6.69 \text { to }-2.94)^{* *}$ & $-7.64(-10.47 \text { to }-4.81)^{* *}$ \\
\hline \multirow[t]{3}{*}{ Concept shifting } & Model 1 & $8.49(3.41 \text { to } 13.56)^{* *}$ & $23.42(15.70 \text { to } 31.13)^{* *}$ \\
\hline & Model 2 & $8.04(2.79 \text { to } 13.28)^{* *}$ & 20.00 (11.92 to 28.08$)^{* *}$ \\
\hline & Model 3 & $7.95(2.72 \text { to } 13.18)^{* *}$ & $21.82(13.57 \text { to } 30.06)^{* *}$ \\
\hline \multirow[t]{3}{*}{ Immediate word recall } & Model 1 & $-1.14(-3.54$ to 1.27$)$ & $-1.60(-4.92$ to 1.73$)$ \\
\hline & Model 2 & $-0.74(-3.19$ to 1.71$)$ & $-1.03(-4.34$ to 2.28$)$ \\
\hline & Model 3 & $-1.23(-3.72$ to 1.25$)$ & $-1.88(-5.28$ to 1.52$)$ \\
\hline \multirow[t]{3}{*}{ Delayed word recall } & Model 1 & $-0.78(-1.57$ to 0.02$)$ & $-1.06(-2.19$ to 0.07$)$ \\
\hline & Model 2 & $-0.65(-1.45$ to 0.16$)$ & $-1.06(-2.18$ to 0.06$)$ \\
\hline & Model 3 & $-0.82(-1.64 \text { to }-0.01)^{*}$ & $-1.35(-2.50 \text { to }-0.20)^{*}$ \\
\hline
\end{tabular}

For information processing speed, immediate word recall, and delayed word recall, higher scores indicate better performance. For concept shifting, lower scores indicate better performance. Model 1: Crude model; Model 2: Model 1 + adjustment for demographic variables, history of smoking, and alcohol intake; Model 3: Model $2+$ adjustment for comorbid conditions (hypertension, history of cardiovascular disease, BMI, and depression). ${ }^{*} P<0.05, * * P<0.01$.

This was virtually unchanged after adjusting for demographic variables, history of smoking and alcohol intake (model 2) and after further adjustments for comorbid conditions (model 3). The association between diabetes and change in delayed word recall from baseline to $\mathrm{F} 1$ and to $\mathrm{F} 2$ was not significant in models 1 and 2, but became modestly significant in the fully adjusted model 3 . In contrast, there were no associations with decline in immediate word recall. To examine the effect of carrying the APOE-e4 allele on the association between diabetes and cognitive change over time, APOE-e4 was included as a covariate in a restricted subgroup of 699 individuals without and 36 with baseline diabetes. Results were similar to the analyses in the full sample (data not shown), except that the effect of diabetes on decline in delayed word recall from baseline to F1 was not statistically significant anymore $(P=0.14)$. However, the magnitude of this effect was similar to the analysis without APOE-e4. The results per cognitive outcome-domain of the fully-adjusted models (without APOE-e4) are presented in Figure 2.1. Decline in information processing speed from baseline to $\mathrm{F} 2$ was three times larger for participants with diabetes compared with those without and decline in set shifting was four times larger. Although participants without diabetes did not decline in delayed word recall, participants with diabetes declined by $14 \%$. In contrast, there were no group differences in change in immediate word recall. 

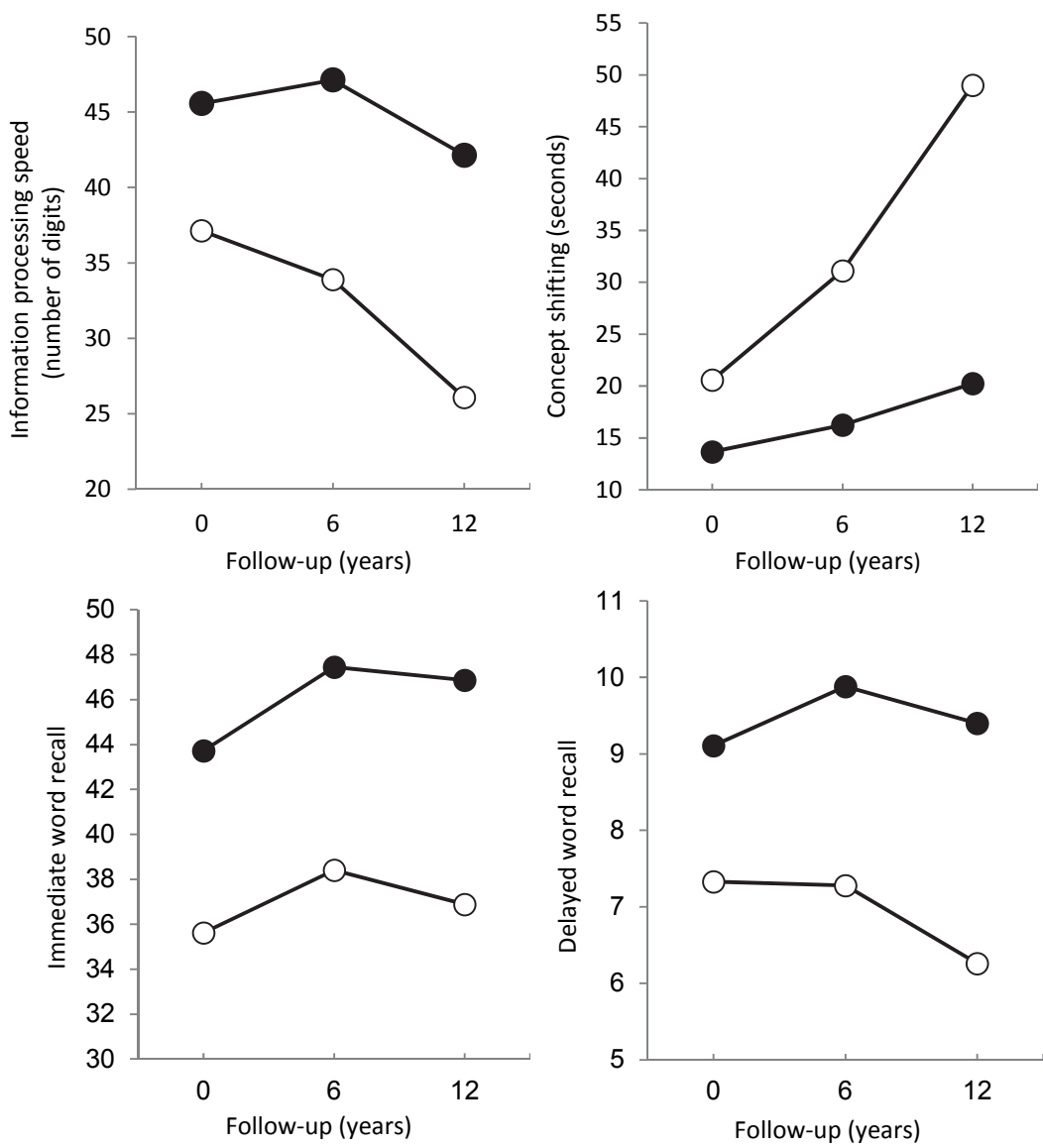

Figure 2.1 Cognitive performance (mean domain score adjusted for demographics, history of smoking, alcohol intake and comorbid conditions) for participants with baseline type 2 diabetes (white circles) and participants without baseline diabetes (black circles) at baseline (0), 6-year follow-up (6), and 12-year follow-up (12). For information processing speed, and immediate and delayed word recall a higher score indicates better performance. For concept shifting, a lower score indicates better performance.

\section{Incident diabetes and 12-year cognitive decline}

Results of the longitudinal analyses are presented in Table 2.3. In crude analyses, participants with incident diabetes at F1 showed a larger decline in information processing speed from baseline to F1 and to F2 (Table 2.3, model 1). This effect was considerably attenuated in the fully adjusted model (model 3 ). No differences were observed between participants who developed diabetes at follow-up and controls. 


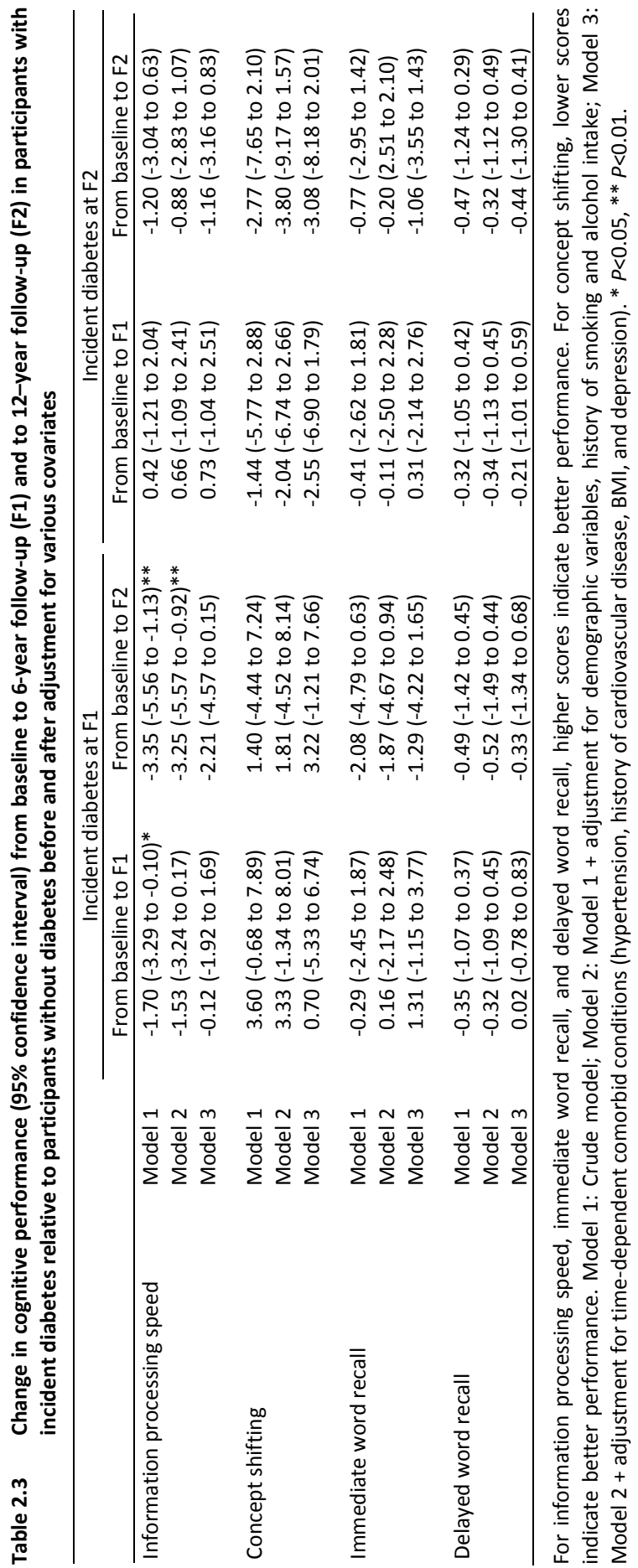




\section{Post-hoc analyses}

To better understand the effects of incident diabetes on cognitive decline, we performed two post-hoc analyses. In the first analysis, the two groups with participants with incident diabetes were pooled $(n=111)$ to increase power. In the fully adjusted model, the incident diabetes group showed a significantly larger decline in information processing speed from baseline to F2 compared with the control group (estimate $=-1.62, P=0.04$ ). There was no significant difference in decline in the other cognitive measures. Next, we explored whether disease exposure time plays a role in the development of cognitive decline. We performed a post-hoc analysis including only diabetic patients and measured the effect of disease duration on cognitive decline. Data on disease duration (based on self-report) were available at baseline for 67 participants with baseline diabetes and at follow-up for 44 participants with incident diabetes at F1 and for 53 participants with incident diabetes at F2. Missing values were replaced with the strata-specific mean of the observed values of disease duration within each of the three diabetes strata (baseline, at F1, at F2). Disease duration was calculated relative to the year of baseline assessment. On average, diabetes had been diagnosed 1,01 $\pm 9,15$ years (mean \pm SD) after baseline assessment. In line with our expectation, we found that, after adjustment for all covariates described previously, increasing disease duration had a significant effect on decline in information processing speed from baseline to 12-year follow-up (estimate=-0.19, $P<0.05)$. We did not find a significant effect of disease duration on immediate recall (estimate $=0.04, P=0.72$ ), delayed recall (estimate $=0.03, P=0.41$ ) or concept shifting (estimate $=0.59, P=0.05$ ).

\section{Discussion}

This study examined the effect of baseline and incident diabetes on change in multiple cognitive functions over a long follow-up period of 12 years. Participants with baseline diabetes performed worse on all cognitive measures at baseline and also showed a three times larger decline in information processing speed and a four times larger decline in executive functions than participants without diabetes. The effect of diabetes on memory was less pronounced. The effects on speed and executive function were largely independent of APOE-e4 genotype status.

Interestingly, no significant effect of incident diabetes at F1 and F2 on cognitive decline was observed, though the coefficients suggested a small effect on decline in information processing speed from baseline to 12-year follow-up. Few previous studies examined the effect of incident diabetes on cognitive decline. ${ }^{26,27}$ The study by Nooyens et al. ${ }^{26}$ was performed in a restricted sample of middle-aged individuals followed for five years. The recent study by Yaffe et al. ${ }^{27}$ used two cognitive measures and was performed in older adults followed for nine years. Though both studies did 
not find an independent effect of incident diabetes on cognitive decline, both point out that the decline in the incident diabetes group was intermediate between the control group and the prevalent diabetes group. In a post-hoc analysis in which we pooled the two groups with incident diabetes at F1 and F2, incident diabetes indeed had a significant effect on decline in information processing speed. This might indicate that our initial model had missed the small differences in cognitive decline between incident diabetic patients and controls. So, diabetes might have a subtle effect on cognition even in the early stages of the disease.

The finding that baseline diabetes had an effect on cognitive decline is in line with numerous previous studies with shorter follow-up duration, ${ }^{8,9}$ although the rate of decline in our study was larger than reported before, and some studies did not find an effect of diabetes on cognitive decline. ${ }^{5-7}$ Given the differences in the effect of baseline diabetes and incident diabetes, it appears that effects of diabetes on cognition take time to evolve, and that profound differences in cognition between people with diabetes and people without diabetes become apparent only in the long run. This is consistent with the finding that incident diabetes only had an effect on a measure of information processing speed, which is known to be most sensitive to diabetes-related cognitive decline ${ }^{28}$ and with the finding that disease duration had a significant effect on decline in information processing speed. These results seem to indicate that duration of disease exposure plays an important role in the development of cognitive decline, which is in line with previous research showing that longer duration of diabetes is associated with increased odds of mild cognitive impairment, ${ }^{29}$ a possible prodromal stage of dementia.

Few studies examined the effect of baseline diabetes on cognitive decline over a comparable follow-up period. ${ }^{10,30-32}$ In the Baltimore Longitudinal Study of Aging, no effect of diabetes on cognitive decline over a 12-year follow-up period was found. However, this study only included men and assessed other cognitive domains, namely visual memory and vocabulary performance. ${ }^{31}$ The Study of Osteoporotic Fractures (SOF) has shown that lack of diabetes was predictive of maintaining optimal cognitive function over 15-year follow-up as opposed to minor cognitive decline in older women. ${ }^{32}$ The Indianapolis-Ibadan Dementia Project and The Atherosclerosis Risk in Communities Studies showed a modest effect of diabetes on change in cognition over a 15 -year and 14-year follow-up period, respectively. ${ }^{10,30}$ Stronger associations might have been missed because measures of general cognitive functioning were used, ${ }^{10}$ which are less sensitive to change, and because the study sample was restricted to middle-aged individuals, ${ }^{30}$ in whom the association between diabetes and cognitive decline might be less obvious. ${ }^{26}$ In contrast, the present study had no upper age restriction and used a comprehensive and validated multicognitive domain test battery.

In line with previous studies ${ }^{33-35}$ we found that diabetes was most strongly related to decline in information processing speed and executive function, although in these studies the follow-up durations were shorter and the age ranges were smaller 
than in our study. In the absence of major cerebrovascular disease or neurodegenerative processes, decline in these domains has been linked to cerebral small vessel disease. ${ }^{36,37}$ Indeed, patients with diabetes have been shown to have more white matter lesions, which have been related to cognitive functioning in these patients. $^{38}$ Furthermore, microvascular disease seems to play a role in the development of dementia in diabetic patients. ${ }^{1,39}$ Other putative factors include hyperglycemia, which can have toxic effects on the brain by glycation of important proteins, ${ }^{1,2}$ and insulin, which inhibits degradation of amyloid- $\beta$ peptide (the main product of the Alzheimer's disease process) by competition for insulin degrading enzyme in the brain. ${ }^{1,2}$

Our study has some limitations. First, the size of the diabetes groups was relatively small due to the population-based nature of MAAS, especially the incident diabetes groups. We are thus likely to have missed more subtle associations between incident diabetes and cognitive decline, especially for the group with incident diabetes at F1 and decline from baseline to F2. Next, participants with diabetes and poorer global cognition were more likely to drop out of the study, which might have led to selection bias. Hence, our results most likely are an underestimation of the "true" effect of diabetes on cognitive decline in the population. However, we partially accounted for this attrition by using mixed models, which does not restrict analysis to study completers. Furthermore, the diagnosis of diabetes was based on self-report or on antidiabetic medication use. As a diagnosis of diabetes is often missed, ${ }^{40}$ some participants with diabetes might have been wrongly assigned to the nondiabetic group. It is unknown how this would have affected our estimates, but it seems unlikely that this accounts for the robust differences in the rate of cognitive decline between groups. Finally, since we did not have data on $\mathrm{HbA} 1 \mathrm{C}$ levels, we were unable to examine the effect of glucose control on cognitive decline.

This study also has a number of important strengths. These include multiple measures of cognitive functions, assessment of separate cognitive domains by a comprehensive neuropsychological test battery, inclusion of important potential confounders and a long follow-up time. Furthermore, we had the opportunity to measure the effect of incident diabetes and comorbid conditions at each follow-up moment.

To conclude, this study indicates that patients with baseline type 2 diabetes show accelerated cognitive decline, particularly in information processing speed and executive function, compared with individuals without diabetes and that patients with incident diabetes show signs of early decline in information processing speed. It seems that disease exposure time plays an important role in the development of cognitive decline. This might provide a window of opportunity for prevention and early treatment of diabetes related cognitive deficits. For this, it is important to assess cognitive status at an early stage of the disease and on a regular basis. Studies that focus on the underlying mechanisms between diabetes and cognitive decline are highly desirable in order to develop adequate treatment for cognitive decline. 


\section{References}

1. Biessels GJ, Staekenborg S, Brunner E, Brayne C, Scheltens P. Risk of dementia in diabetes mellitus: a systematic review. Lancet Neurol 2006;5:64-74

2. Lu FP, Lin KP, Kuo HK. Diabetes and the risk of multi-system aging phenotypes: a systematic review and meta-analysis. PLoS One 2009;4:e4144

3. Strachan MW, Reynolds RM, Marioni RE, Price JF. Cognitive function, dementia and type 2 diabetes mellitus in the elderly. Nature Rev Endocrinol 2011;7:108-14

4. CFAS M. Pathological correlates of late-onset dementia in a multicentre, community-based population in England and Wales. Neuropathology Group of the Medical Research Council Cognitive Function and Ageing Study (MRC CFAS). Lancet 2001;357:169-75

5. van den Berg E, de Craen AJ, Biessels GJ, Gussekloo J, Westendorp RG. The impact of diabetes mellitus on cognitive decline in the oldest of the old: a prospective population-based study. Diabetologia 2006;49:2015-23

6. Fischer AL, de Frias CM, Yeung SE, Dixon RA. Short-term longitudinal trends in cognitive performance in older adults with type 2 diabetes. J Clin Exp Neuropsychol 2009;31:809-22

7. van den Berg E, Reijmer YD, de Bresser J, Kessels RP, Kappelle LJ, Biessels GJ. A 4 year follow-up study of cognitive functioning in patients with type 2 diabetes mellitus. Diabetologia 2010;53:58-65

8. Fontbonne A, Berr C, Ducimetiere P, Alperovitch A. Changes in cognitive abilities over a 4-year period are unfavorably affected in elderly diabetic subjects: results of the Epidemiology of Vascular Aging Study. Diabetes Care 2001;24:366-70

9. Yaffe K, Blackwell T, Kanaya AM, Davidowitz N, Barrett-Connor E, Krueger K. Diabetes, impaired fasting glucose, and development of cognitive impairment in older women. Neurology 2004;63: 658-63

10. Wessels AM, Lane KA, Gao S, Hall KS, Unverzagt FW, Hendrie HC. Diabetes and cognitive decline in elderly African Americans: a 15-year follow-up study. Alzheimers Dement 2011;7:418-24

11. Reijmer YD, van den Berg E, Ruis C, Kappelle LJ, Biessels GJ. Cognitive dysfunction in patients with type 2 diabetes. Diabetes Metab Res Rev 2010;26:507-19

12. Jolles J, Houx PJ, van Boxtel MP, Ponds RW, (Eds.). Maastricht Aging Study: Determinants of cognitive aging. Maastricht, Neuropsych Publishers, 1995

13. Metsemakers JF, Hoppener P, Knottnerus JA, Kocken RJ, Limonard CB. Computerized health information in The Netherlands: a registration network of family practices. $\mathrm{Br} J$ Gen Pract 1992;42:102-6

14. Logroscino $\mathrm{G}$, Kang JH, Grodstein F. Prospective study of type 2 diabetes and cognitive decline in women aged 70-81 years. BMJ 2004;328:548

15. Goldman N, Lin IF, Weinstein M, Lin YH. Evaluating the quality of self-reports of hypertension and diabetes. J Clin Epidemiol 2003;56:148-54

16. WHO Expert Committee on Problems Related to Alcohol Consumption. Problems Related to Alcohol Consumption: Technical Report Series 650. Geneva, World Health Organization, 1980

17. World Health Organization-International Society of Hypertension Guidelines for the Management of Hypertension. J Hypertens 1999;17:151-83

18. Arrindell WA, Ettema JHM. SCL-90. Een multidimensionele psychopathologie indicator [A multidimensional indicator of psychopathology] Lisse, the Netherlands: Swets \& Zeitlinger; 1986

19. Bekers O, op den Buijsch RA, de Vries JE, Wijnen PA, van Dieijen-Visser MP. Capillary electrophoretic detection in apolipoprotein E genotyping. Electrophoresis 2002;23:1878-81

20. Slooter AJ, Tang MX, van Duijn CM, et al. Apolipoprotein E epsilon4 and the risk of dementia with stroke. A population-based investigation. JAMA 1997;277:818-21

21. De Bie SE. Standaardvragen 1987: Voorstellen voor uniformering van vraagstellingen naar achtergrondkenmerken en interviews. Leiden, Leiden University Press, 1987

22. Van der Elst W, van Boxtel MP, van Breukelen GJ, Jolles J. Rey's verbal learning test: normative data for 1855 healthy participants aged 24-81 years and the influence of age, sex, education, and mode of presentation. J Int Neuropsychol Soc 2005;11:290-302

23. Van der Elst W, Van Boxtel MP, Van Breukelen GJ, Jolles J. The Concept Shifting Test: adult normative data. Psychol Assess 2006;18:424-32 
24. van der Elst W, van Boxtel MP, van Breukelen GJ, Jolles J. The Letter Digit Substitution Test: normative data for 1,858 healthy participants aged 24-81 from the Maastricht Aging Study (MAAS): influence of age, education, and sex. J Clin Exp Neuropsychol 2006;28:998-1009

25. Folstein MF, Folstein SE, McHugh PR. "Mini-mental state". A practical method for grading the cognitive state of patients for the clinician. J Psychiatr Res 1975;12:189-98

26. Nooyens AC, Baan CA, Spijkerman AM, Verschuren WM. Type 2 diabetes and cognitive decline in middle-aged men and women: the Doetinchem Cohort Study. Diabetes Care 2010;33:1964-9

27. Yaffe K, Falvey C, Hamilton N, et al. Diabetes, glucose control, and 9-year cognitive decline among older adults without dementia. Arch Neurol 2012;69:1170-5

28. Messier C. Impact of impaired glucose tolerance and type 2 diabetes on cognitive aging. Neurobiol Aging 2005;26 Suppl 1:26-30

29. Roberts RO, Geda YE, Knopman DS, et al. Association of duration and severity of diabetes mellitus with mild cognitive impairment. Arch Neurol 2008;65: 1066-73

30. Knopman DS, Mosley TH, Catellier DJ, Coker LH. Fourteen-year longitudinal study of vascular risk factors, APOE genotype, and cognition: the ARIC MRI Study. Alzheimers Dement 2009;5:207-14

31. Robertson-Tchabo EA, Arenberg D, Tobin JD, Plotz JB. A longitudinal study of cognitive performance in noninsulin dependent (type II) diabetic men. Exp Gerontol 1986;21:459-67

32. Barnes DE, Cauley JA, Lui LY, et al. Women who maintain optimal cognitive function into old age. J Am Geriatr Soc 2007;55:259-64

33. Takahashi $\mathrm{Y}$, Iseki $\mathrm{C}$, Wada $\mathrm{M}$, et al. Impaired glucose metabolism slows executive function independent of cerebral ischemic lesions in Japanese elderly: the Takahata study. Intern Med 2011;50:1671-8

34. Bourdel-Marchasson I, Lapre E, Laksir H, Puget E. Insulin resistance, diabetes and cognitive function: consequences for preventative strategies. Diabetes Metab 2010;36:173-81

35. Arvanitakis Z, Wilson RS, Bienias JL, Evans DA, Bennett DA. Diabetes mellitus and risk of Alzheimer disease and decline in cognitive function. Arch Neurol 2004;61:661-6

36. Prins ND, van Dijk EJ, den Heijer T, et al. Cerebral small-vessel disease and decline in information processing speed, executive function and memory. Brain 2005;128:2034-41

37. Umemura $\mathrm{T}$, Kawamura $\mathrm{T}$, Umegaki $\mathrm{H}$, et al. Endothelial and inflammatory markers in relation to progression of ischaemic cerebral small-vessel disease and cognitive impairment: a 6-year longitudinal study in patients with type 2 diabetes mellitus. J Neurol Neurosurg Psychiatry 2011;82:1186-94

38. Manschot SM, Brands AM, van der Grond J, et al. Brain magnetic resonance imaging correlates of impaired cognition in patients with type 2 diabetes. Diabetes 2006;55:1106-13

39. Sonnen JA, Larson EB, Brickell K, et al. Different patterns of cerebral injury in dementia with or without diabetes. Arch Neurol 2009;66:315-22

40. Cowie CC, Rust KF, Ford ES, et al. Full accounting of diabetes and pre-diabetes in the U.S. population in 1988-1994 and 2005-2006. Diabetes Care 2009;32:287-94 


\section{Supplemental data}

\section{Patient recruitment flow chart}

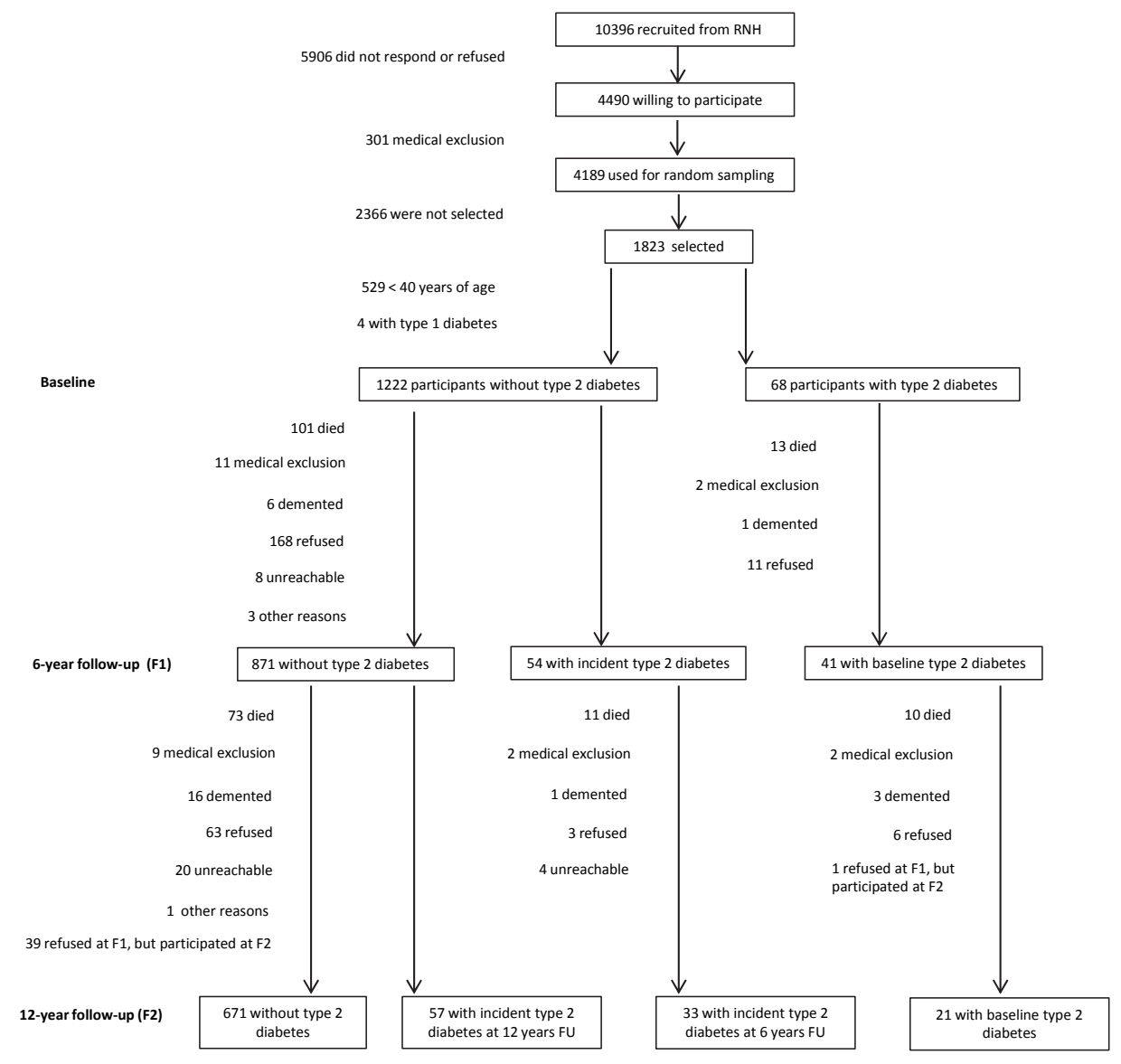




\section{Chapter 3}

ASSOCIATIONS OF ADVANCED GLYCATION

END-PRODUCTS WITH COGNITIVE FUNCTIONS IN

INDIVIDUALS WITH AND WITHOUT TYPE 2 DIABETES:

THE MAASTRICHT STUDY

PJJ Spauwen, MGA van Eupen, S Köhler, CDA Stehouwer, FRJ Verhey, CJH van der Kallen, SJS Sep, A Koster, NC Schaper, PC Dagnelie, CG Schalkwijk, MT Schram, MPJ van Boxtel The Journal of Clinical Endocrinology and Metabolism 2015;100:951-60 


\section{Abstract}

\section{Context}

Advanced glycation end-products (AGEs) are thought to be involved in the pathogenesis of Alzheimer's disease. AGEs are products resulting from non-enzymatic chemical reactions between reduced sugars and proteins, which accumulate during natural aging, and their accumulation is accelerated in hyperglycemic conditions such as type 2 diabetes mellitus.

\section{Objective}

Examine associations between AGEs and cognitive functions

\section{Design, setting, participants}

This study was performed as part of The Maastricht study, a population-based cohort study, in which, by design, 215 participants ( $28.1 \%$ ) had type 2 diabetes mellitus.

\section{Main outcome measures}

We examined associations of skin autofluorescence (SAF) ( $n=764)$, an overall estimate of skin AGEs, and specific plasma protein-bound AGEs $(n=781)$ with performance on tests for global cognitive functioning, information processing speed, verbal memory (immediate and delayed word recall), and response inhibition.

\section{Results}

After adjustment for demographics, diabetes, smoking, alcohol, waist circumference, total cholesterol/HDL cholesterol ratio, triglycerides, and lipid-lowering medication use, higher SAF was significantly associated with worse delayed word recall (regression coefficient $b=-0.44$, $P=0.04)$, and response inhibition $(b=0.03, P=0.04)$. After further adjustment for systolic blood pressure, cardiovascular disease, estimated glomerular filtration rate, and depression, associations were attenuated (delayed word recall: $b=-0.38, P=0.07$; response inhibition: $b=0.02, P=0.07)$. Higher pentosidine levels were associated with worse global cognitive functioning $(b=-0.61, P=0.04)$ after full adjustment, but other plasma AGEs were not. Associations did not differ between individuals with and without diabetes.

\section{Conclusion}

We found inverse associations of SAF, a noninvasive marker for tissue AGES, with cognitive performance, which were attenuated after adjustment for vascular risk factors and depression. 


\section{Introduction}

Dementia is highly prevalent worldwide and the number of people with dementia is expected to increase rapidly. ${ }^{1}$ The most common cause of dementia is Alzheimer's disease (AD), which is characterized by progressive decline in cognitive function, more specifically memory loss. It is associated with the presence and the accumulation of neurofibrillary tangles and amyloid plaques in the brain. ${ }^{2}$ Although the etiology of dementia has not been elucidated, the relationship between, on the one hand, type 2 diabetes mellitus and other cardiovascular risk factors including hypertension, obesity and smoking, and the development of dementia and cognitive decline on the other has become more evident. ${ }^{3,4}$

Advanced glycation end-products (AGEs), which are products resulting from nonenzymatic chemical reactions between reduced sugars and proteins, ${ }^{5}$ are thought to be involved in the pathogenesis of AD. ${ }^{6}$ AGEs accumulate during natural aging and their accumulation is accelerated in hyperglycemic conditions such as type 2 diabetes. ${ }^{5,7}$ Long-lived proteins, including $\beta$-amyloid $(A \beta)$, have been found to be modified by AGEs and a recent study has shown that the formation of AGE-modified $A \beta$ exacerbates the toxicity of $A B .^{8}$ AGEs are present in both neurofibrillary tangles and senile plaques of patients with $A D^{9}$ and the receptor for AGE (RAGE) appears to be involved in the transport of amyloid peptides through the blood-brain barrier. ${ }^{10}$ In addition, $A D$ patients with type 2 diabetes seem to have more severe $A D$ pathology and higher AGE levels in the brain compared with those with $A D$ alone. ${ }^{9}$

AGEs can be measured in plasma (circulating AGEs) or estimated in tissue using a relative simple non-invasive measurement of skin autofluorescence (SAF), a method based on the fluorescent properties of some AGEs. ${ }^{11}$ SAF has been suggested to be a simple alternative to invasive measurement of AGE accumulation and has shown to be correlated with fluorescent (pentosidine) and even non-fluorescent plasma AGEs (N(epsilon)-(carboxymethyl)lysine (CML) and N(epsilon)-(carboxyethyl)lysine (CEL)) in biopsy-derived skin tissue. ${ }^{11}$ In addition, SAF has recently been shown to be associated with lower grey matter volume ${ }^{12}$ and it may therefore be hypothesized that SAF indicates AGE accumulation in other tissue, like the brain, as well. Furthermore, SAF may reflect $A G E$ accumulation more accurately than plasma proteins as intracellular glycation is thought to be the major local source of AGEs and not all AGEs may end up in the circulation. ${ }^{13,14}$

Although there is evidence that AGEs might be associated with dementia and cognitive decline, research on the relationship between AGEs and cognitive functions is scarce. Yaffe et al. ${ }^{15}$ have shown that the AGE pentosidine, measured in its free form in urine, was associated with a greater 9-year cognitive decline in older people independent of diabetes status. Furthermore, Chen et al. ${ }^{16}$ showed that higher levels of serum AGEs were cross-sectionally associated with Mild Cognitive Impairment $(\mathrm{MCl})$ in diabetic patients. No study to date has investigated the relationship between SAF and cognitive performance on multiple cognitive domains. One recent study has 
shown a cross-sectional association between higher SAF and a higher likelihood of cognitive impairment, but did not examine associations with separate cognitive functions. $^{12}$

Examination of the association between AGEs and cognitive function might provide a marker for cognitive impairment and might increase our knowledge about the etiology of cognitive decline and dementia. Therefore, the aim of our study was to examine the associations of SAF and plasma AGEs with performance on a range of cognitive tests in participants from The Maastricht Study, a population-based cohort study. Next, we investigated whether or not these associations were different in participants with and without type 2 diabetes.

\section{Methods}

\section{Study population}

In this study, we used data from The Maastricht Study, an observational prospective population-based cohort study. The rationale and methodology have been described previously. ${ }^{17}$ In brief, the study focuses on the etiology, pathophysiology, complications and comorbidities of type 2 diabetes mellitus and is characterized by an extensive phenotyping approach. Eligible for participation were all individuals aged between 40 and 75 years and living in the southern part of the Netherlands. Participants were recruited through mass media campaigns and from the municipal registries and the regional Diabetes Patient Registry via mailings. Recruitment was stratified according to known type 2 diabetes status for reasons of efficiency. The present report includes cross-sectional data from the first 866 participants, who completed the baseline survey between November 2010 and March 2012. The examinations of each participant were performed within a time window of three months. The study has been approved by the institutional medical ethical committee (NL31329.068.10) and the Netherlands Health Council under the Dutch "Law for Population Studies" (Permit 131088-105234-PG). All participants gave written informed consent.

\section{Skin autofluorescence}

All participants were asked to refrain from smoking and caffeine at least 3 hours before the measurements. A light meal (breakfast and (or) lunch), low in fat content, was allowed. SAF was measured with the AGE Reader ${ }^{\mathrm{TM}}$ (DiagnOptics Technologies BV, Groningen, the Netherlands). The AGE reader is a desktop device that uses the characteristic fluorescent properties of certain AGEs to estimate the level of AGE accumulation in the skin. Technical details of this non-invasive method have been described more extensively elsewhere. ${ }^{11}$ In short, the AGE Reader illuminates a skin 
surface of $4 \mathrm{~cm}^{2}$ guarded against surrounding light, with an excitation wavelength range of $300-420 \mathrm{~nm}$, with a peak excitation of $370 \mathrm{~nm}$. SAF was calculated as the ratio between the emission light from the skin in the wavelength range of $420-600 \mathrm{~nm}$ (fluorescence) and excitation light that is reflected by the skin (300-420 nm), multiplied by 100 and expressed in arbitrary units (AU). Participants were asked not to use any sunscreen or self-browning creams on their lower arms within 2 days before the measurement. SAF was measured at room temperature in a semi-dark environment while participants were at rest in a seated position. The forearm of a participant was positioned on top of the device, as described by the manufacturer. The mean of three consecutive measurements was used in the analyses. Reproducibility was assessed in 14 individuals without diabetes (6 males; 32.2 \pm 7.1 years). The intraclass correlation coefficient (ICC) of three intra-individual consecutive SAF measurements was $0.83(95 \% \mathrm{Cl} 0.65-0.94)$. SAF was calculated off-line by automated analysis using AGE Reader software, version 2.3, and was observerindependent. There were no significant differences between fasting and non-fasting measurements (mean difference $=0.01 \mathrm{AU}, P=0.73$ ). Reproducibility in individuals with type 2 diabetes has been evaluated previously ${ }^{11}$ with an overall Altman error percentage of $5.03 \%$ for measurements taken over a single day. Skin pigmentation is known to influence the measurement of SAF. ${ }^{18}$ Therefore, in participants with darkcolored skin with a reflectance of $6-10 \%$, a validated reflectance dependent correction was made by the software. ${ }^{18}$ Measurements in participants with dark-colored skin and a mean reflectance below $6 \%$ are considered unreliable and are therefore not used to calculate SAF by the software. Therefore, these participants were automatically excluded. Additionally, a single SAF value above $10 \mathrm{AU}$ was considered as unreliable; these individual measurements $(n=3)$ were manually excluded and the mean of the remaining two measurements was used in analyses.

\section{Analysis of protein bound AGEs and lysine in plasma}

Plasma AGEs were measured in EDTA samples obtained from fasting venous blood, which were stored at $-80^{\circ} \mathrm{C}$ until analysis. Protein-bound pentosidine was quantified using HPLC with fluorescence detection, as described in detail elsewhere. ${ }^{19}$ Intra- and interassay coefficients of variation (CVs), as analyzed in this study, were 6.5 and $7.8 \%$ for pentosidine, respectively. Protein-bound CML, CEL and lysine were quantified using UPLC MS/MS. ${ }^{14}$. Intra- and interassay CVs were 4.5 and $6.7 \%$ for $\mathrm{CML}, 6.2$ and $10.3 \%$ for CEL and 5.0 and $5.3 \%$ for lysine. Concentrations of protein-bound pentosidine, CML and CEL were adjusted for levels of lysine and expressed as $\mathrm{nmol} / \mathrm{mmol}$ lysine.

\section{Assessment of cognitive function}

A concise battery (30 $\mathrm{min}$ ) of cognitive tests was used to assess cognitive functioning. ${ }^{17}$ An a priori selection of these cognitive tests was used in the current 
study. Since diabetes is strongly linked to AGE accumulation, we have chosen tests that each represent cognitive domains (i.e. information processing speed, verbal memory, and executive functions) which are often used and have been shown to be most sensitive to effects of diabetes. ${ }^{20}$ Global cognitive functioning was measured by the Mini-Mental State Examination (MMSE) (score range $0-30) .{ }^{21}$ Verbal memory was assessed with the Visual Verbal Word Learning Test. ${ }^{22}$ In this test, 15 words are presented in five subsequent trials, followed by a recall phase immediately after each trial (immediate recall) (score range 0-75), and a delayed recall phase 20 minutes thereafter (delayed recall) (score range 0-15). Response inhibition was measured with the Stroop Color Word Test. ${ }^{23}$ The variable of interest was the interference measure expressed in seconds. The Letter-Digit Substitution Test ${ }^{24}$ was used to measure information processing speed. Participants were instructed to match digits to letters as quickly as possible within 90 seconds.

\section{Covariates}

History of cardiovascular disease, diabetes duration, smoking status (never, former, current) and alcohol consumption were assessed by questionnaire. ${ }^{17}$ Participants were regarded as having a history of cardiovascular disease if they reported to have had a: myocardial infarction, and/or cerebrovascular infarction or hemorrhage, and/or percutaneous artery angioplasty of, or vascular surgery on, the coronary, abdominal, peripheral or carotid arteries. Alcohol consumption was classified into three categories: non-consumers, low-consumers ( $\leq 7$ glasses per week for females and $\leq 14$ glasses per week for males) and high-consumers (>7 glasses per week for females and $>14$ glasses per week for males). Lipid-lowering, antihypertensive, and glucoselowering medication use were assessed during a medication interview where generic name, dose and frequency were registered. ${ }^{17}$ Waist circumference, glycosylated hemoglobin A1c ( $\mathrm{HbA1c})$, glucose levels, total and high-density lipoprotein (HDL) cholesterol, creatinine, and triglycerides were determined as described elsewhere. ${ }^{17}$ Estimated glomerular filtration rate (eGFR) was estimated according to the CKD-EPI (Chronic Kidney Disease Epidemiology Collaboration) equation. ${ }^{25}$ Office blood pressure was determined three times on the right arm after a 10-minute rest period, using a non-invasive blood pressure monitor (Omron 705IT, Omron, Japan). ${ }^{17}$

To determine glucose metabolism, all participants (except those who use insulin) underwent a standardized 7-point oral glucose tolerance test after an overnight fast as previously described. ${ }^{17}$ Glucose metabolism was defined according to the World Health Organization 2006 criteria into normal glucose tolerance (NGT), impaired fasting glucose (IFG), impaired glucose tolerance (IGT), and type 2 diabetes. $^{26}$ Additionally, individuals without type 1 diabetes and on diabetes medication were considered as having type 2 diabetes. ${ }^{17}$ For this study we defined having either IFG or IGT as impaired glucose metabolism (IGM). 
Level of education was assessed during the cognitive assessment and was classified into 8 categories commonly used in the Netherlands ${ }^{27}:(1)$ no education, (2) primary education, (3) lower vocational education, (4) intermediate general secondary education, (5) intermediate vocational education, (6) higher general secondary education, (7) higher vocational education, (8) university. For this study, three groups were created for educational level: low (level 1 to 3), intermediate (level 4 to 6), and high (level 7-8). Depression was assessed by the Mini International Neuropsychiatric Interview. $^{17,28}$

\section{Statistical analysis}

Analyses were conducted using the SPSS software, version 20 for Mac OSX (SPSS Inc). Differences between tertiles of SAF were tested using analysis of variance for continuous variables and Chi-square tests for categorical variables. Multiple linear regression analysis was used to estimate the association of SAF and of plasma AGEs with cognitive performance, adjusted for different sets of covariates in separate models. In Model 1, we adjusted for age, which is a known predictor of AGE accumulation and cognitive performance and therefore considered as an important potential confounder. In Model 2, we added other potential important confounders: sex, educational level, and diabetes (yes/no). In Model 3, we additionally adjusted for cardiovascular risk factors that have been previously associated with higher AGE accumulation and with cognitive performance, and therefore may be potential confounders (i.e. smoking, alcohol, waist circumference, total cholesterol/HDL cholesterol ratio, triglycerides, and lipid-lowering medication use). Finally, in Model 4, we adjusted for variables that could be potential mediators of the associations between AGEs and cognition, because they may be caused by higher AGE accumulation and may cause cognitive impairment (i.e. systolic blood pressure, cardiovascular disease, depression, and eGFR). Interaction effects were tested to examine whether the association of SAF and plasma AGEs with cognitive performance differed between participants with and without diabetes. Pentosidine levels and response inhibition scores were log-transformed before regression analysis, because they were positively skewed. A $P$-value of $<0.05$ was considered statistically significant in two-sided tests.

\section{Results}

\section{Sample}

Four individuals with type 1 diabetes and 4 participants who did not undergo cognitive assessment were excluded. Of the remaining 858 participants, we additionally excluded individuals with missing data on the independent variables SAF $(n=31)$ or 
plasma AGEs $(n=19)$, or on potential confounders $(n=63)$. This resulted in 764 individuals available for complete case analyses with SAF and 781 individuals for complete case analyses with plasma AGEs. Participants excluded due to missing values were more likely to have diabetes, to use insulin, had higher levels of HbA1c and SAF, and had lower scores for cognitive performance (global cognitive functioning, information processing speed, immediate word recall, and response inhibition $(P<0.05))$. There were no differences in other characteristics (data not shown).

Characteristics of the 764 participants included for analyses with SAF are shown in Table 3.1, stratified according to tertiles of SAF. Of these, 215 participants $(28.1 \%)$ had type 2 diabetes, of whom 35 (16.3\%) participants were newly diagnosed at study entry. Median diabetes duration was 7.0 years (interquartile range=3.0-11.0), and mean $\mathrm{HbA} 1 \mathrm{c}$ level was $6.9 \%(\mathrm{SD}= \pm 0.8)$. Of the 549 participants without diabetes, 126 participants ( $16.5 \%$ of the total sample) had IGM.

Mean scores for cognitive tests in the total sample were $28.9(S D= \pm 1.2)$ for global cognitive functioning, 48.8 digits $(S D= \pm 9.3)$ for information processing speed, 45.4 words $(S D= \pm 9.7)$ for immediate word recall, and 9.5 words $(S D= \pm 2.9$ ) for delayed word recall. The median score for response inhibition was 41.7 seconds (IQR=31.9-55.9). Participants with type 2 diabetes had significantly lower scores on all cognitive measures compared with those with NGT $(P<0.001)$ after adjustment for age, while participants with IGM did not perform significantly worse on any cognitive test compared with those with NGT ( $P>0.10$ for all cognitive measures). We therefore combined participants with NGT with those with IGM for the interaction analyses.

Tertiles of SAF were significantly associated with age, educational level, glucose metabolism status, smoking status, alcohol consumption, waist circumference, systolic blood pressure, history of cardiovascular disease, $\mathrm{HbA1c}$ level, antihypertensive medication use, lipid-lowering medication use, glucose-lowering medication use, eGFR, pentosidine level, and cognitive functions (Table 3.1). 
Table 3.1 Characteristics of the study group ( $n=764)$, stratified by tertiles of skin autofluorescence

\begin{tabular}{|c|c|c|c|c|}
\hline Characteristic & Low $(n=254)$ & Middle ( $n=255)$ & High $(n=255)$ & $P$-value \\
\hline Skin autofluorescence (AU), mean (SD) & $2.17(0.20)$ & $2.65(0.12)$ & $3.27(0.38)$ & - \\
\hline Age $(y)$, mean (SD) & $54.9(8.7)$ & $60.2(7.3)$ & $63.8(6.9)$ & $<0.001$ \\
\hline Sex: Male, n (\%) & $129(50.8)$ & $138(54.1)$ & $154(60.4)$ & 0.09 \\
\hline Educational level, low/middle/high, n (\%) & $\begin{array}{c}26 / 98 / 130 \\
(10.2 / 38.6 / 51.2)\end{array}$ & $\begin{array}{c}31 / 106 / 118 \\
(12.2 / 41.6 / 46.3)\end{array}$ & $\begin{array}{c}66 / 108 / 81 \\
(25.9 / 42.4 / 31.8)\end{array}$ & $<0.001$ \\
\hline Glucose metabolism status, NGT/IGM/T2DM, n (\%) & $\begin{array}{c}175 / 41 / 38 \\
(68.9 / 16.1 / 15.0)\end{array}$ & $\begin{array}{c}153 / 38 / 64 \\
(60.0 / 14.9 / 25.1)\end{array}$ & $\begin{array}{c}95 / 47 / 113 \\
(37.3 / 18.4 / 44.3)\end{array}$ & $<0.001$ \\
\hline Smoking status, never/former/current, n (\%) & $\begin{array}{c}97 / 133 / 24 \\
(38.2 / 52.4 / 9.4)\end{array}$ & $\begin{array}{c}80 / 135 / 40 \\
(31.4 / 52.9 / 15.7)\end{array}$ & $\begin{array}{c}62 / 139 / 54 \\
(24.3 / 54.5 / 21.2)\end{array}$ & 0.001 \\
\hline Alcohol consumption, none/low/high, n (\%) & $\begin{array}{c}39 / 141 / 74 \\
(15.4 / 55.5 / 29.1)\end{array}$ & $\begin{array}{c}34 / 126 / 95 \\
(13.4 / 49.4 / 37.3)\end{array}$ & $\begin{array}{c}57 / 136 / 62 \\
(22.4 / 53.3 / 24.3)\end{array}$ & 0.01 \\
\hline Waist circumference $(\mathrm{cm})$, mean (SD) & $95.2(12.1)$ & $96.0(3.0)$ & $100.0(15.1)$ & $<0.001$ \\
\hline Systolic blood pressure (mmHg), mean (SD) & $134.1(16.9)$ & $137.4(19.2)$ & $139.6(20.1)$ & 0.01 \\
\hline Antihypertensive medication, $\mathrm{n}(\%)$ & $68(26.8)$ & $95(37.4)$ & $140(55.1)$ & $<0.001$ \\
\hline Lipid-lowering medication, n (\%) & $56(22.0)$ & $88(34.5)$ & $130(51.0)$ & $<0.001$ \\
\hline \multicolumn{5}{|l|}{ Glucose-lowering medication } \\
\hline None/oral ${ }^{\mathrm{a}}$ /insulin, $\mathrm{n}(\%)$ & $\begin{array}{c}229 / 22 / 3 \\
(90.2 / 8.7 / 1.2)\end{array}$ & $\begin{array}{c}207 / 42 / 6 \\
(81.2 / 16.5 / 2.4)\end{array}$ & $\begin{array}{c}160 / 66 / 29 \\
(62.7 / 25.9 / 11.4)\end{array}$ & $<0.001$ \\
\hline Cardiovascular disease, n (\%) & $31(12.2)$ & $36(14.1)$ & $70(27.5)$ & $<0.001$ \\
\hline HbA1c (\%), mean (SD) & $5.8(0.5)$ & $5.9(0.7)$ & $6.3(0.9)$ & $<0.001$ \\
\hline Triglycerides (mmol/I), median (IQR) & $1.20(0.82-1.73)$ & $1.24(0.85-1.76)$ & $1.23(0.88-1.88)$ & $0.59 *$ \\
\hline Total cholesterol/HDL cholesterol, mean (SD) & $4.24(1.26)$ & $4.24(1.24)$ & $4.18(1.27)$ & 0.84 \\
\hline eGFR ml/min $/ 1.73 \mathrm{~m}^{2}$, mean (SD) & $89.1(13.84)$ & $85.83(13.27)$ & $79.86(14.15)$ & $<0.001$ \\
\hline Depression, n (\%) & $8(3.1)$ & $9(3.5)$ & $14(5.5)$ & 0.36 \\
\hline Pentosidine $\mathrm{nmol} / \mathrm{mmol} \mathrm{LYS}$, median (IQR) & $0.45(0.37-0.53)$ & $0.47(0.38-0.55)$ & $0.50(0.40-0.60)$ & $<0.001^{*}$ \\
\hline CML nmol/mmol LYS, mean (SD) & $74.8(14.2)$ & $74.6(14.3)$ & $73.8(15.9)$ & 0.67 \\
\hline CEL nmol/mmol LYS, mean (SD) & $34.0(10.4)$ & $33.9(10.4)$ & $34.1(10.0)$ & 0.95 \\
\hline Global cognitive functioning ${ }^{\mathrm{b}}$, mean (SD) & $29.1(1.2)$ & $29.0(1.2)$ & $28.7(1.3)$ & 0.001 \\
\hline Information processing speed ${ }^{b}$, mean (SD) & $51.5(9.3)$ & $48.7(8.7)$ & $46.1(9.2)$ & $<0.001$ \\
\hline Immediate word recall ${ }^{\mathrm{b}}$, mean (SD) & $48.0(9.3)$ & $46.4(9.1)$ & $41.8(9.7)$ & $<0.001$ \\
\hline Delayed word recall ${ }^{\mathrm{b}}$, mean (SD) & $10.3(2.8)$ & $9.7(2.8)$ & $8.5(2.9)$ & $<0.001$ \\
\hline${\text { Response inhibition }{ }^{\mathrm{c}} \text {, median (IQR) }}$ & $37.8(29.0-48.5)$ & $41.1(33.0-54.1)$ & $48.1(35.5-63.5)$ & $<0.001 *$ \\
\hline
\end{tabular}

${ }^{a}$ Two participants in this group used glucagon-like peptide-1 receptor agonists in addition to oral glucose-lowering medication. ${ }^{b}$ Higher scores indicate better performance. ${ }^{C}$ Lower scores indicate better performance. ${ }^{*} P$-values were derived from analysis of variance with log-transformed outcomes. SD, standard deviation; IQR, interquartile rang; IGM, impaired glucose metabolism; T2DM, type 2 diabetes mellitus; HbA1c, glycated hemoglobin; HDL, high-density lipoprotein; eGFR, estimated glomerular filtration rate; CML, N(epsilon)-(carboxymethyl)lysine; CEL, N(epsilon)-(carboxyethyl)lysine.

\section{Skin autofluorescence and cognitive performance}

In unadjusted analyses a higher SAF level was significantly associated with worse performance on all cognitive measures (regression coefficient $b=-0.44, P<0.001$ for global cognitive functioning; $b=-4.69, P<0.001$ for information processing speed; $b=-5.28, P<0.001$ for immediate word recall; $b=-1.51, P<0.001$ for delayed word recall; $\mathrm{b}=0.11, P<0.001$ for response inhibition). After adjustment for age (Table 3.2, Model 1), SAF was still significantly associated with all cognitive measures. 
Table 3.2 Adjusted association between skin autofluorescence and cognitive performance

\begin{tabular}{|c|c|c|c|}
\hline & b (regression coefficient) & $95 \% \mathrm{Cl}$ & $P$-value \\
\hline \multicolumn{4}{|c|}{ Global cognitive functioning $^{\mathrm{a}}$} \\
\hline Model 1 & $-0.25^{c}$ & -0.43 to -0.06 & 0.01 \\
\hline Model 2 & -0.10 & -0.28 to 0.08 & 0.29 \\
\hline Model 3 & -0.08 & -0.26 to 0.11 & 0.42 \\
\hline Model 4 & -0.08 & -0.27 to 0.11 & 0.41 \\
\hline \multicolumn{4}{|c|}{ Information processing speed ${ }^{a}$} \\
\hline Model 1 & -1.73 & -3.03 to -0.42 & 0.01 \\
\hline Model 2 & -0.42 & -1.68 to 0.84 & 0.52 \\
\hline Model 3 & -0.13 & -1.42 to 1.17 & 0.84 \\
\hline Model 4 & 0.07 & -1.24 to 1.38 & 0.91 \\
\hline \multicolumn{4}{|c|}{ Immediate Word recall $^{\mathrm{a}}$} \\
\hline Model 1 & -2.58 & -3.96 to -1.20 & $<0.001$ \\
\hline Model 2 & -1.39 & -2.68 to -0.10 & 0.03 \\
\hline Model 3 & -1.15 & -2.46 to 0.17 & 0.09 \\
\hline Model 4 & -0.97 & -2.30 to 0.36 & 0.15 \\
\hline \multicolumn{4}{|c|}{ Delayed Word recall ${ }^{\mathrm{a}}$} \\
\hline Model 1 & -0.74 & -1.16 to -0.32 & 0.001 \\
\hline Model 2 & -0.48 & -0.88 to -0.08 & 0.02 \\
\hline Model 3 & -0.44 & -0.85 to -0.03 & 0.04 \\
\hline Model 4 & -0.38 & -0.79 to 0.04 & 0.07 \\
\hline \multicolumn{4}{|c|}{ Response inhibition $^{\mathrm{b}}$} \\
\hline Model 1 & 0.05 & 0.03 to 0.08 & $<0.001$ \\
\hline Model 2 & 0.03 & 0.00 to 0.05 & 0.03 \\
\hline Model 3 & 0.03 & 0.00 to 0.05 & 0.04 \\
\hline Model 4 & 0.02 & 0.00 to 0.05 & 0.07 \\
\hline
\end{tabular}

${ }^{a}$ Higher scores indicate better performance (MMSE score for global cognitive functioning, number of digits for information processing speed, number of words for total and delayed word recall). ${ }^{b}$ Lower scores indicate better performance. Scores for response inhibition (seconds) are log-transformed. ${ }^{\mathrm{C}} \mathrm{A}$ regression coefficient of -0.25 indicates that one unit increase in skin autofluorescence level is associated with a decrease of 0.25 points on a test for global cognitive functioning. Model 1: Adjustment for age; Model 2: Model 1 + adjustments for sex, diabetes, and educational level; Model 3: Model 2 + adjustments for smoking, alcohol consumption, waist circumference, total cholesterol/HDL cholesterol ratio, triglycerides, and lipid-lowering medication use; Model 4: Model 3 + adjustments for systolic blood pressure, cardiovascular disease, depression, and estimated glomerular filtration rate.

After further adjustment for sex, educational level, and diabetes (Table 3.2, Model 2), SAF was still significantly associated with immediate and delayed word recall, and with response inhibition, but not with global cognitive functioning and information processing speed. After further adjustment for smoking status, alcohol consumption, waist circumference, total cholesterol/HDL cholesterol ratio, triglycerides, and lipid-lowering medication use (Model 3), the association of SAF with immediate word recall was attenuated and became non-significant. Associations with delayed word recall and response inhibition were attenuated, but remained statistically significant. Associations of SAF with delayed word recall and response inhibition were attenuated and became non-significant after further adjustment for systolic blood pressure, cardiovascular disease, eGFR, and depression (Table 3.2, Model 4). In a post-hoc analysis, we additionally adjusted the associations for antihypertensive medication (yes/no) use and glucose-lowering medication use 
(yes/no). The association with delayed recall increased somewhat $(b=-0.41, P=0.05)$, whereas the association with response inhibition was somewhat attenuated $(b=0.02$, $P=0.10)$. Other associations remained virtually unchanged $(b=-0.10, P=0.32$ for global cognitive functioning; $0.04, P=0.96$ for speed; $b=-1.08, P=0.12$ for immediate word recall).

Interactions between SAF and diabetes on cognitive measures were not significant (Model 4: regression coefficient $b$ for interaction $=-0.31 ; P=0.09$ for global cognitive functioning; $b=-0.09, P=0.95$ for speed; $b=0.41, P=0.75$ for immediate word recall; $b=0.12, P=0.77$ for delayed word recall; $b=0.02, P=0.35$ for response inhibition).

\section{Plasma AGEs and cognitive performance}

In unadjusted analyses, higher pentosidine levels were significantly associated with worse immediate and delayed word recall, and response inhibition $(b=-4.67, P=0.04$ for immediate word recall; $b=-1.73, P=0.01$ for delayed word recall; $b=0.09, P=0.04$ for response inhibition). In adjusted models (Table 3.3), these associations became nonsignificant, whereas the association between pentosidine and global cognitive functioning became stronger and significant (Table 3.3, Model 4). After additional adjustment for antihypertensive and glucose-lowering medication use, these results were largely unchanged (for global cognitive functioning: $b=-0.63, P=0.03$; for speed: -1.46, $P=0.47$; for immediate word recall: $b=-3.61, P=0.08$; for delayed word recall: $b=-1.11, P=0.08$; for response inhibition: $b=0.01, P=0.72)$. Interactions between pentosidine and diabetes on cognitive measures were not significant (Model 4: $b$ for interaction=0.09, $P=0.87$ for global cognitive functioning; $b=-3.30, P=0.38$ for information processing speed; $b=-2.77, P=0.47$ for immediate word recall; $b=-0.23$, $P=0.85$ for delayed word recall; $b=0.07, P=0.38$ for response inhibition).

Plasma CML and CEL were not significantly associated with any cognitive measures after adjustment for confounders (Supplementary Tables S3.1 and S3.2, respectively). We found no significant interactions between $\mathrm{CML}$ and diabetes on any of the cognitive measures (data not shown). There was a significant interaction between CEL and diabetes on global cognitive functioning (b for interaction $=0.03$, $P=0.003$ ), but not on the other cognitive measures. Stratified analyses showed that CEL was only associated with global cognitive functioning in participants without diabetes $(b=-0.01, P=0.003)$, but not in participants with diabetes $(b=0.01, P=0.13)$. 
Table 3.3 Adjusted association between plasma pentosidine and cognitive performance

\begin{tabular}{|c|c|c|c|}
\hline & $\mathrm{b}$ (regression coefficient) & $95 \% \mathrm{Cl}$ & $P$-value \\
\hline \multicolumn{4}{|c|}{ Global cognitive functioning $^{a}$} \\
\hline Model 1 & $-0.14^{\mathrm{c}}$ & -0.70 to 0.42 & 0.62 \\
\hline Model 2 & -0.46 & -1.00 to 0.07 & 0.09 \\
\hline Model 3 & -0.51 & -1.05 to 0.04 & 0.07 \\
\hline Model 4 & -0.61 & -1.17 to -0.04 & 0.04 \\
\hline \multicolumn{4}{|c|}{ Information processing speed ${ }^{a}$} \\
\hline Model 1 & 1.37 & -2.56 to 5.30 & 0.50 \\
\hline Model 2 & -1.29 & -4.98 to 2.40 & 0.49 \\
\hline Model 3 & -1.38 & -5.15 to 2.40 & 0.47 \\
\hline Model 4 & -1.37 & -5.30 to 2.56 & 0.49 \\
\hline \multicolumn{4}{|c|}{ Immediate Word Recall $^{\mathrm{a}}$} \\
\hline Model 1 & -0.15 & -4.33 to 4.03 & 0.95 \\
\hline Model 2 & -2.63 & -6.40 to 1.15 & 0.17 \\
\hline Model 3 & -3.39 & -7.23 to 0.45 & 0.08 \\
\hline Model 4 & -3.45 & -7.45 to 0.55 & 0.09 \\
\hline \multicolumn{4}{|c|}{ Delayed Word Recall ${ }^{a}$} \\
\hline Model 1 & -0.44 & -1.71 to 0.82 & 0.49 \\
\hline Model 2 & -1.00 & -2.18 to 0.18 & 0.10 \\
\hline Model 3 & -1.05 & -2.25 to 0.14 & 0.09 \\
\hline Model 4 & -1.02 & -2.26 to 0.23 & 0.11 \\
\hline \multicolumn{4}{|c|}{ Response inhibition $^{\mathrm{b}}$} \\
\hline Model 1 & -0.01 & -0.09 to 0.06 & 0.74 \\
\hline Model 2 & 0.04 & -0.04 to 0.11 & 0.33 \\
\hline Model 3 & 0.03 & -0.04 to 0.10 & 0.44 \\
\hline Model 4 & 0.02 & -0.06 to 0.10 & 0.65 \\
\hline
\end{tabular}

${ }^{a}$ Higher scores indicate better performance (MMSE score for global cognitive functioning, number of digits for information processing speed, number of words for total and delayed word recall). ${ }^{b}$ Lower scores indicate better performance. Scores for pentosidine and response inhibition (seconds) are log-transformed. ${ }^{c} \mathrm{~A}$ regression coefficient of -0.14 indicates that one unit increase in log-transformed pentosidine level is associated with a decrease of 0.14 points on a test for global cognitive functioning. Model 1: Adjustment for age; Model 2: Model 1 + adjustments for sex, diabetes, and educational level; Model 3: Model $2+$ adjustments for smoking, alcohol consumption, waist circumference, total cholesterol/HDL cholesterol ratio, triglycerides, and lipid-lowering medication use; Model 4: Model 3 + adjustments for systolic blood pressure, cardiovascular disease, depression, and estimated glomerular filtration rate.

\section{Sensitivity analysis}

Z-scores for SAF and pentosidine were calculated for each individual as their value for AGE level minus the mean and divided by the SD of the study sample. In a sensitivity analysis we excluded participants with AGEs Z-scores higher than 3 or lower than -3 to examine if our results would change. Six participants had a Z-score higher than 3. When these six participants were excluded from analyses, the associations of SAF with immediate word recall (Model 4: $b=-0.99, P=0.17$ ) and delayed word recall did not change (Model 4: $b=-0.41, P=0.07$ ), while the association of SAF with response inhibition $(b=0.02, P=0.26)$ was attenuated. Other associations remained nonsignificant. For analyses with pentosidine, we excluded sixteen participants with Z-score larger than 3 . The association of pentosidine with global cognitive functioning 
(Model 4: $b=-0.52, P=0.15)$ was attenuated and became non-significant. Other associations remained non-significant.

\section{Discussion}

This is the first study to examine the association of SAF, as an estimate of tissue AGE accumulation, and plasma AGEs with multiple cognitive functions. Our results indicate that SAF is inversely associated with memory, although cardiovascular risk factors seem to be involved in the association. In addition we found associations between SAF and response inhibition and between pentosidine and global cognitive functioning which should be interpreted with caution, because these associations were attenuated after excluding some influential cases. Therefore, in our study the association between SAF and memory was most robust. As not all AGEs may end up in the circulation, ${ }^{13,14}$ our results indicate that SAF may be a better marker for AGE accumulation in brain tissue than plasma AGEs.

The associations between SAF or plasma pentosidine and cognitive performance were not significantly different between individuals with and without type 2 diabetes. While our study may not have enough power to detect significant differences, our results are in line with previous research demonstrating no interaction between diabetes and urinary pentosidine on cognitive decline. ${ }^{15}$

Accumulation of AGEs in the brain has been linked to $A D$ by increasing inflammation, oxidative stress, and subsequent neuronal dysfunction. ${ }^{6}$ These mechanisms may be involved in the development of cognitive impairment. Interestingly, in our study the strongest association was found between SAF and delayed word recall, which is the best neuropsychological predictor of AD. ${ }^{29}$ However, associations of SAF with cognitive functions were attenuated after adjustment for potential confounders/mediators. Several (cardio)vascular risk factors may confound the relationship between AGEs and cognitive impairment.

Diabetes has been associated with higher accumulation of $\mathrm{AGEs}^{5}$ and cognitive decline. ${ }^{30}$ In addition, research has shown that obesity increases the risk of developing dementia and cognitive impairment, possibly in part through the accumulation of AGEs. ${ }^{31,32}$ In addition, higher levels of lipids, which are involved in the formation of AGEs, ${ }^{33}$ may also increase the risk of cognitive impairment. ${ }^{34}$ However, in our sample lipids were not associated with SAF.

In addition to in vivo production, AGEs have been found in cigarettes, ${ }^{35}$ and therefore smoking can increase AGE levels and may increase the risk of cognitive impairment. ${ }^{36}$ High alcohol consumption may increase oxidative stress, and thereby AGE levels, ${ }^{37}$ and can affect cognitive function. ${ }^{38}$ However, in our sample, high SAF level was not associated with high alcohol consumption.

Some other factors may mediate the association between AGEs and cognitive impairment, e.g. systolic blood pressure, depression, kidney functioning, and 
cardiovascular disease. AGE accumulation may contribute to vascular stiffening, by collagen crosslinking of the vascular wall and thereby leading to (systolic) hypertension, ${ }^{39}$ which has been associated with lower cognitive performance and lower total brain matter volume. ${ }^{40}$ Additionally, depression has been both associated with vascular stiffness ${ }^{41}$ and cognitive decline, ${ }^{42}$ but in our sample depression was not associated with SAF. Furthermore, the kidney metabolizes and removes plasma AGEs and is a site for accumulation of AGEs. Research has shown that a decreased GFR is associated both with higher plasma AGE levels ${ }^{43}$ and more cognitive decline. ${ }^{44} \mathrm{~A}$ decreased GFR may therefore mediate the association between AGEs and cognitive impairment, but may also predict AGE accumulation. Finally, AGEs can lead to cardiovascular disease, through mechanisms discussed previously (e.g. vascular stiffness and hypertension), which can in turn lead to cognitive impairment. ${ }^{45}$

It is important to note that some of the variables we adjusted for, e.g. GFR, systolic blood pressure and cardiovascular disease, could be part of the causal pathway from AGEs to cognitive impairment. Therefore, we may have overadjusted our associations, resulting in a reduction in the potential total causal effect of AGEs on cognition by controlling for an intermediate variable. ${ }^{46}$ Moreover, since participants that were excluded from analyses (due to missing values) had higher SAF levels and lower cognitive scores, our results may be an underestimation of the true association of AGEs and cognitive functions.

Furthermore, the associations between AGE accumulation and cognition may be stronger in individuals with cognitive impairment. Studies that found associations with plasma AGEs mostly investigated this in individuals with cognitive impairment or dementia. ${ }^{16,47}$ In addition, in one recent study, SAF was associated with cognitive impairment (score <-1.5 standard deviations in any domain from age, sex, and education adjusted norms). ${ }^{12}$ Stronger associations could emerge in longitudinal data in which participants develop cognitive decline or impairment. ${ }^{15}$

\section{Strengths and limitations}

Our study has several strengths. A major strength is that it is the first study to associate SAF and several plasma AGEs with separate cognitive domains. In addition, we were able to adjust for multiple important potential confounders. Our study also has some limitations. First, due to the cross-sectional design we were not able to address causal relationships. However, longitudinal data are not available yet. Second, SAF may not only reflect skin AGEs, but also non-AGE skin fluorophores. ${ }^{11}$ Nevertheless, results of previous research support the use of SAF as a marker for skin tissue AGEs. ${ }^{11}$ Third, it remains unclear if SAF is an accurate reflectance of the level of AGE accumulation in the brain. However, research has shown that higher SAF is associated with lower brain volume. ${ }^{12}$ 


\section{Conclusion}

We found significant inverse associations between SAF, a potential marker of tissue AGEs, and memory. These were attenuated and became non-significant after adjustment for vascular risk factors and depression. In addition, we found associations of SAF with response inhibition and of pentosidine with global cognitive functioning, albeit not robust. Our results may suggest that AGEs are involved in the development of cognitive decline, particularly memory decline, and possibly in part through the action of vascular risk factors. More longitudinal research is needed to examine the effect of tissue and plasma AGEs on decline in separate cognitive domains. 


\section{References}

1. Prince M, Bryce R, Albanese E, Wimo A, Ribeiro W, Ferri CP. The global prevalence of dementia: a systematic review and metaanalysis. Alzheimers Dement 2013;9:63-75 e2

2. LaFerla FM, Oddo S. Alzheimer's disease: Abeta, tau and synaptic dysfunction. Trends Mol Med 2005;11:170-6

3. Daviglus ML, Bell CC, Berrettini W, et al. NIH state-of-the-science conference statement: Preventing Alzheimer's disease and cognitive decline. NIH Consens State Sci Statements 2010;27:1-30

4. Barnes DE, Yaffe K. The projected effect of risk factor reduction on Alzheimer's disease prevalence. Lancet Neurol 2011;10:819-28

5. Brownlee M. Advanced protein glycosylation in diabetes and aging. Annu Rev Med 1995;46:223-34

6. Li J, Liu D, Sun L, Lu Y, Zhang Z. Advanced glycation end products and neurodegenerative diseases: mechanisms and perspective. J Neurol Sci 2012;317:1-5

7. Schalkwijk CG, Brouwers O, Stehouwer CD. Modulation of insulin action by advanced glycation endproducts: a new player in the field. Horm Metab Res 2008;40:614-9

8. Li XH, Du LL, Cheng XS, et al. Glycation exacerbates the neuronal toxicity of beta-amyloid. Cell Death Dis 2013;4:e673

9. Valente T, Gella A, Fernandez-Busquets X, Unzeta M, Durany N. Immunohistochemical analysis of human brain suggests pathological synergism of Alzheimer's disease and diabetes mellitus. Neurobiol Dis 2010;37:67-76

10. Candela P, Gosselet F, Saint-Pol J, et al. Apical-to-basolateral transport of amyloid-beta peptides through blood-brain barrier cells is mediated by the receptor for advanced glycation end-products and is restricted by P-glycoprotein. J Alzheimers Dis 2010;22:849-59

11. Meerwaldt R, Graaff R, Oomen $\mathrm{PH}$, et al. Simple non-invasive assessment of advanced glycation endproduct accumulation. Diabetologia 2004;47:1324-30

12. Moran C, Munch G, Forbes JM, et al. Type 2 diabetes mellitus, skin autofluorescence and brain atrophy. Diabetes 2015;64:279-83

13. Shinohara M, Thornalley PJ, Giardino I, et al. Overexpression of glyoxalase-I in bovine endothelial cells inhibits intracellular advanced glycation endproduct formation and prevents hyperglycemia-induced increases in macromolecular endocytosis. J Clin Invest 1998;101:1142-7

14. Hanssen NM, Engelen L, Ferreira I, et al. Plasma levels of advanced glycation endproducts Nepsilon(carboxymethyl)lysine, Nepsilon-(carboxyethyl)lysine, and pentosidine are not independently associated with cardiovascular disease in individuals with or without type 2 diabetes: the Hoorn and CODAM studies. J Clin Endocrinol Metab 2013;98:E1369-73

15. Yaffe K, Lindquist K, Schwartz AV, et al. Advanced glycation end product level, diabetes, and accelerated cognitive aging. Neurology 2011;77:1351-6

16. Chen G, Cai L, Chen B, et al. Serum level of endogenous secretory receptor for advanced glycation end products and other factors in type 2 diabetic patients with mild cognitive impairment. Diabetes care 2011;34:2586-90

17. Schram MT, Sep SJ, van der Kallen CJ, et al. The Maastricht Study: an extensive phenotyping study on determinants of type 2 diabetes, its complications and its comorbidities. Eur J Epidemiol 2014;29: 439-51

18. Koetsier $\mathrm{M}$, Nur $\mathrm{E}$, Chunmao $\mathrm{H}$, et al. Skin color independent assessment of aging using skin autofluorescence. Opt Express 2010;18:14416-29

19. Scheijen JL, van de Waarenburg MP, Stehouwer CD, Schalkwijk CG. Measurement of pentosidine in human plasma protein by a single-column high-performance liquid chromatography method with fluorescence detection. J Chromatogr B Analyt Technol Biomed Life Sci 2009;877:610-4

20. Kodl CT, Seaquist ER. Cognitive dysfunction and diabetes mellitus. Endocr Rev 2008;29:494-511

21. Folstein MF, Folstein SE, McHugh PR. "Mini-mental state". A practical method for grading the cognitive state of patients for the clinician. J Psychiatr Res 1975;12:189-98

22. Van der Elst W, van Boxtel MP, van Breukelen GJ, Jolles J. Rey's verbal learning test: normative data for 1855 healthy participants aged 24-81 years and the influence of age, sex, education, and mode of presentation. J Int Neuropsychol Soc 2005;11:290-302 
23. Van der Elst W, Van Boxtel MP, Van Breukelen GJ, Jolles J. The Stroop color-word test: influence of age, sex, and education; and normative data for a large sample across the adult age range. Assessment 2006;13:62-79

24. van der Elst W, van Boxtel MP, van Breukelen GJ, Jolles J. The Letter Digit Substitution Test: normative data for 1,858 healthy participants aged 24-81 from the Maastricht Aging Study (MAAS): influence of age, education, and sex. J Clin Exp Neuropsychol 2006;28:998-1009

25. Levey AS, Stevens LA, Schmid CH, et al. A new equation to estimate glomerular filtration rate. Ann Intern Med 2009;150:604-12

26. World Health Organization. Definition and Diagnosis of Diabetes Mellitus and Intermediate Hyperglycemia: Report of a WHO/IDF Consultation. Geneva, World Health Organization, 2006

27. De Bie SE. Standaardvragen 1987: Voorstellen voor uniformering van vraagstellingen naar achtergrondkenmerken en interviews. Leiden, Leiden University Press, 1987

28. Sheehan DV, Lecrubier $\mathrm{Y}$, Sheehan $\mathrm{KH}$, et al. The Mini-International Neuropsychiatric Interview (M.I.N.I.): the development and validation of a structured diagnostic psychiatric interview for DSM-IV and ICD-10. J Clin Psychiatry 1998;59 Suppl 20:22-33;quiz 4-57

29. Gainotti G, Quaranta D, Vita MG, Marra C. Neuropsychological predictors of conversion from mild cognitive impairment to Alzheimer's disease. J Alzheimers Dis 2014;38:481-95

30. Spauwen PJ, Kohler S, Verhey FR, Stehouwer CD, van Boxtel MP. Effects of type 2 diabetes on 12-year cognitive change: results from the maastricht aging study. Diabetes Care 2013;36:1554-61

31. Luchsinger JA, Gustafson DR. Adiposity and Alzheimer's disease. Curr Opin Clin Nutr Metab Care 2009;12:15-21

32. Yoon DH, Choi SH, Yu JH, Ha JH, Ryu SH, Park DH. The relationship between visceral adiposity and cognitive performance in older adults. Age Ageing 2012;41:456-61

33. Fu MX, Requena JR, Jenkins AJ, Lyons TJ, Baynes JW, Thorpe SR. The advanced glycation end product, Nepsilon-(carboxymethyl)lysine, is a product of both lipid peroxidation and glycoxidation reactions. J Biol Chem 1996;271:9982-6

34. Reitz C, Tang MX, Luchsinger J, Mayeux R. Relation of plasma lipids to Alzheimer disease and vascular dementia. Arch Neurol 2004;61:705-14

35. Cerami C, Founds $\mathrm{H}$, Nicholl I, et al. Tobacco smoke is a source of toxic reactive glycation products. Proc Natl Acad Sci U S A 1997;94:13915-20

36. Sabia S, Marmot M, Dufouil C, Singh-Manoux A. Smoking history and cognitive function in middle age from the Whitehall II study. Arch Intern Med 2008;168:1165-73

37. Kalousova M, Zima T, Popov $\mathrm{P}$, et al. Advanced glycation end-products in patients with chronic alcohol misuse. Alcohol Alcohol 2004;39:316-20

38. Xu G, Liu X, Yin $Q$, Zhu W, Zhang R, Fan X. Alcohol consumption and transition of mild cognitive impairment to dementia. Psychiatry Clin Neurosci 2009;63:43-9

39. Zieman SJ, Kass DA. Advanced glycation endproduct crosslinking in the cardiovascular system: potential therapeutic target for cardiovascular disease. Drugs 2004;64:459-70

40. Nagai M, Hoshide S, Ishikawa J, Shimada K, Kario K. Ambulatory blood pressure as an independent determinant of brain atrophy and cognitive function in elderly hypertension. J Hypertens 2008;26:1636-41

41. Tiemeier H, Breteler MM, van Popele NM, Hofman A, Witteman JC. Late-life depression is associated with arterial stiffness: a population-based study. J Am Geriatr Soc 2003;51:1105-10

42. Kohler S, van Boxtel MP, van Os J, et al. Depressive symptoms and cognitive decline in communitydwelling older adults. J Am Geriatr Soc 2010;58:873-9

43. Semba RD, Ferrucci L, Fink JC, et al. Advanced glycation end products and their circulating receptors and level of kidney function in older community-dwelling women. Am J Kidney Dis 2009;53:51-8

44. Buchman AS, Tanne D, Boyle PA, Shah RC, Leurgans SE, Bennett DA. Kidney function is associated with the rate of cognitive decline in the elderly. Neurology 2009;73:920-7

45. Breteler MM, Claus JJ, Grobbee DE, Hofman A. Cardiovascular disease and distribution of cognitive function in elderly people: the Rotterdam Study. BMJ 1994;308:1604-8

46. Schisterman EF, Cole SR, Platt RW. Overadjustment bias and unnecessary adjustment in epidemiologic studies. Epidemiology 2009;20:488-95

47. Meli M, Perier C, Ferron C, et al. Serum pentosidine as an indicator of Alzheimer's disease. J Alzheimers Dis 2002;4:93-6 


\section{Supplemental data}

Table S3.1 Adjusted association between plasma CML and cognitive performance

\begin{tabular}{|c|c|c|c|}
\hline & b (regression coefficient) & $95 \% \mathrm{Cl}$ & $P$-value \\
\hline \multicolumn{4}{|c|}{ Global cognitive functioning ${ }^{a}$} \\
\hline Model 1 & $0.008^{\mathrm{c}}$ & 0.002 to 0.014 & 0.01 \\
\hline Model 2 & 0.002 & -0.004 to 0.008 & 0.48 \\
\hline Model 3 & 0.002 & -0.004 to 0.008 & 0.56 \\
\hline Model 4 & 0.001 & -0.005 to 0.008 & 0.65 \\
\hline \multicolumn{4}{|c|}{ Information processing speed ${ }^{\mathrm{a}}$} \\
\hline Model 1 & 0.059 & 0.019 to 0.100 & 0.004 \\
\hline Model 2 & 0.011 & -0.028 to 0.049 & 0.59 \\
\hline Model 3 & 0.009 & -0.032 to 0.051 & 0.66 \\
\hline Model 4 & 0.011 & -0.032 to 0.054 & 0.62 \\
\hline \multicolumn{4}{|c|}{ Immediate Word recall $^{a}$} \\
\hline Model 1 & 0.065 & 0.022 to 0.107 & 0.003 \\
\hline Model 2 & 0.012 & -0.028 to 0.052 & 0.55 \\
\hline Model 3 & -0.008 & -0.051 to 0.034 & 0.70 \\
\hline Model 4 & -0.009 & -0.053 to 0.035 & 0.69 \\
\hline \multicolumn{4}{|c|}{ Delayed Word recall } \\
\hline Model 1 & 0.017 & 0.004 to 0.030 & 0.01 \\
\hline Model 2 & 0.004 & -0.008 to 0.017 & 0.51 \\
\hline Model 3 & 0.000 & -0.013 to 0.014 & 0.96 \\
\hline Model 4 & 0.001 & -0.013 to 0.014 & 0.93 \\
\hline \multicolumn{4}{|c|}{ Response inhibition $^{\mathrm{b}}$} \\
\hline Model 1 & 0.000 & -0.001 to 0.001 & 0.45 \\
\hline Model 2 & 0.001 & 0.000 to 0.001 & 0.09 \\
\hline Model 3 & 0.001 & 0.000 to 0.001 & 0.15 \\
\hline Model 4 & 0.001 & 0.000 to 0.001 & 0.23 \\
\hline
\end{tabular}

${ }^{a}$ Higher scores (MMSE score for global cognitive functioning, number of digits for information processing speed, number of words for total and delayed word recall) indicate better performance. ${ }^{b}$ Lower scores indicate better performance. Scores for pentosidine and response inhibition (seconds) are log-transformed. ${ }^{\mathrm{C}} \mathrm{A}$ regression coefficient of 0.008 indicates that one unit increase in $\mathrm{N}$ (epsilon)-(carboxymethyl)lysine (CML) level is associated with an increase of 0.008 points on a test for global cognitive functioning. Model 1: Adjustment for age; Model 2: Model $1+$ adjustments for sex, diabetes, and educational level; Model 3: Model 2 + adjustments for smoking, alcohol use, waist circumference, total cholesterol/HDL cholesterol ratio, triglycerides, and lipid-lowering medication use; Model 4: Model 3 + adjustments for systolic blood pressure, cardiovascular disease, depression, and estimated glomerular filtration rate. 
Table S3.2 Adjusted association between plasma CEL and cognitive performance

\begin{tabular}{|c|c|c|c|}
\hline & b (regression coefficient) & $95 \% \mathrm{Cl}$ & $P$-value \\
\hline \multicolumn{4}{|c|}{ Global cognitive functioning ${ }^{a}$} \\
\hline Model 1 & $-0.008^{c}$ & -0.016 to 0.001 & 0.07 \\
\hline Model 2 & -0.004 & -0.012 to 0.004 & 0.28 \\
\hline Model 3 & -0.004 & -0.012 to 0.004 & 0.35 \\
\hline Model 4 & -0.004 & -0.012 to 0.004 & 0.36 \\
\hline \multicolumn{4}{|c|}{ Information processing speed ${ }^{\mathrm{a}}$} \\
\hline Model 1 & -0.002 & -0.060 to 0.056 & 0.94 \\
\hline Model 2 & 0.028 & -0.027 to 0.082 & 0.32 \\
\hline Model 3 & 0.026 & -0.030 to 0.082 & 0.36 \\
\hline Model 4 & 0.025 & -0.032 to 0.081 & 0.39 \\
\hline \multicolumn{4}{|c|}{ Immediate Word Recall } \\
\hline Model 1 & -0.019 & -0.081 to 0.043 & 0.54 \\
\hline Model 2 & 0.005 & -0.051 to 0.061 & 0.87 \\
\hline Model 3 & 0.003 & -0.054 to 0.060 & 0.92 \\
\hline Model 4 & 0.002 & -0.055 to 0.060 & 0.94 \\
\hline \multicolumn{4}{|c|}{ Delayed Word Recall ${ }^{\mathrm{a}}$} \\
\hline Model 1 & 0.001 & -0.017 to 0.020 & 0.88 \\
\hline Model 2 & 0.007 & -0.011 to 0.024 & 0.45 \\
\hline Model 3 & 0.005 & -0.013 to 0.023 & 0.60 \\
\hline Model 4 & 0.005 & -0.013 to 0.023 & 0.59 \\
\hline \multicolumn{4}{|c|}{ Response inhibition $^{\mathrm{b}}$} \\
\hline Model 1 & 0.001 & 0.000 to 0.002 & 0.04 \\
\hline Model 2 & 0.001 & -0.001 to 0.002 & 0.31 \\
\hline Model 3 & 0.001 & -0.001 to 0.002 & 0.29 \\
\hline Model 4 & 0.001 & 0.000 to 0.002 & 0.25 \\
\hline
\end{tabular}

${ }^{\text {a }}$ Higher scores (MMSE score for global cognitive functioning, number of digits for information processing speed, number of words for total and delayed word recall) indicate better performance. ${ }^{b}$ Lower scores indicate better performance. Scores for pentosidine and response inhibition (seconds) are log-transformed. ${ }^{c} \mathrm{~A}$ regression coefficient of -0.008 indicates that one unit increase in $\mathrm{N}$ (epsilon)-(carboxyethyl)lysine (CEL) level is associated with a decrease of 0.008 points on a test for global cognitive functioning. Model 1: Adjustment for age; Model 2: Model $1+$ adjustments for sex, diabetes, and educational level; Model 3: Model $2+$ adjustments for smoking, alcohol use, waist circumference, total cholesterol/HDL cholesterol ratio, triglycerides, and lipid-lowering medication use; Model 4: Model 3 + adjustments for systolic blood pressure, cardiovascular disease, depression, and estimated glomerular filtration rate. 


\section{Chapter 4}

BOTH LOW AND HIGH 24-HOUR DIASTOLIC

BLOOD PRESSURE ARE ASSOCIATED WITH WORSE

COGNITIVE PERFORMANCE IN TYPE 2 DIABETES:

THE MAASTRICHT STUDY

PJJ Spauwen, MPJ van Boxtel, FRJ Verhey, S Köhler, SJS Sep, A Koster, PC Dagnelie, RMA Henry, NC Schaper, CJH van der Kallen, MT Schram, AA Kroon,

CDA Stehouwer 


\section{Abstract}

\section{Objective}

Hypertension and diabetes are both risk factors for cognitive decline and individuals with both might have an especially high risk. We therefore examined linear and nonlinear (quadratic) associations of 24-hour blood pressure with cognitive performance in participants with and without type 2 diabetes mellitus. We also tested the association of nocturnal dipping status with cognitive performance.

\section{Research, design and methods}

This study was performed as part of The Maastricht Study, an ongoing population-based cohort study. Cross-sectional associations of 24-hour blood pressure $(n=713$, of whom 201 had type 2 diabetes) and nocturnal dipping status ( $n=686$, of whom 196 had type 2 diabetes) with performance on tests for global cognitive functioning, information processing speed, verbal memory (immediate and delayed word recall), and response inhibition were tested using linear regression analysis and adjusted for demographics, vascular risk factors, cardiovascular disease, depression, and lipid-modifying and antihypertensive medication use.

\section{Results}

After full adjustment we found quadratic (inverted U-shaped) associations of 24-hour diastolic blood pressure (DBP) with information processing speed ( $b$ for quadratic term $=-0.0267, P<0.01$ ) and memory (immediate word recall: $b=-0.0180, P<0.05$; delayed word recall: $b=-0.0076$, $P<0.01$ ) in participants with diabetes, but not in those without. No clear pattern was found for dipping status.

\section{Conclusions}

This study shows that both low and high 24-hour DBP are associated with poorer performance on tests of information processing speed and memory in individuals with type 2 diabetes. 


\section{Introduction}

Type 2 diabetes is associated with cognitive decline and dementia, ${ }^{1,2}$ but the pathological mechanisms underlying these associations are not yet clear. Proposed mechanisms include the role of vascular risk factors, such as hypertension. Some studies have shown an association of hypertension with cognitive dysfunction and Alzheimer's disease in individuals with diabetes. ${ }^{3,4}$ In addition, diabetes and hypertension seem to interact in their effect on cognitive decline ${ }^{5}$ and dementia, ${ }^{6}$ indicating that individuals with both diabetes and hypertension may have an especially high risk of cognitive decline. Other studies, however, did not confirm these results. ${ }^{7,8}$

Above-mentioned studies ${ }^{5-8}$ used office measurements as an estimate of blood pressure exposure. However, office blood pressure measurement is less accurate than 24-hour ambulatory blood pressure measurement (ABPM) and cannot capture important physiological phenomena such as the normal decrease of blood pressure during sleep (so-called 'dipping'). Indeed, ambulatory blood pressure has shown to be superior to office blood pressure in predicting progression of cerebrovascular disease and cognitive decline. ${ }^{9}$ In addition, non-dipping, i.e. the blunting of the physiological blood pressure reduction during sleep, as well as extreme dipping, i.e. excessive reduction in blood pressure during sleep, have been associated with cerebrovascular damage and lower cognitive performance. ${ }^{10-12}$ Non-dipping is more prevalent in type 2 diabetes, ${ }^{13}$ and as both diabetes and abnormal dipping patterns have been associated with lower cognitive performance, one may speculate that individuals with diabetes who show abnormal dipping in nocturnal blood pressure are especially at risk to develop cognitive impairment.

To our knowledge, there are no studies to date that have addressed the question whether ambulatory blood pressure and dipping parameters are associated with multiple cognitive domains in type 2 diabetes. Therefore, we studied associations between 24-hour ambulatory blood pressure and cognitive performance stratified by diabetes status. Since previous studies have shown quadratic relationships between blood pressure and cognitive functions, ${ }^{14,15}$ we examined both linear and quadratic associations. In addition, we examined the association of non-dipping and extreme dipping in blood pressure during sleep with cognitive performance in individuals with and without type 2 diabetes. Based on the above, we hypothesized that associations between 24-hour ambulatory blood pressure, non-dipping, and extreme dipping on the one hand and cognitive functions on the other are stronger in individuals with type 2 diabetes compared with those without diabetes. 


\section{Research design and methods}

\section{Study population and design}

In this study, we used data from The Maastricht Study, an observational prospective population-based cohort study. The rationale and methodology have been described previously. $^{16}$ In brief, the study focuses on the etiology, pathophysiology, complications and comorbidities of type 2 diabetes mellitus and is characterized by an extensive phenotyping approach. Eligible for participation were all individuals aged between 40 and 75 years and living in the southern part of the Netherlands. Participants were recruited through mass media campaigns and from the municipal registries and the regional Diabetes Patient Registry via mailings. Recruitment was stratified according to known type 2 diabetes status for reasons of efficiency. The present report includes cross-sectional data from the first 866 participants, who completed the baseline survey between November 2010 and March 2012. The examinations of each participant were performed within a time window of three months. The study has been approved by the institutional medical ethical committee (NL31329.068.10) and the Netherlands Health Council under the Dutch "Law for Population Studies" (Permit 131088-105234-PG). All participants gave written informed consent.

\section{Glucose metabolism status}

To determine glucose metabolism, all participants (except those who use insulin) underwent a standardized 7-point oral glucose tolerance test after an overnight fast as previously described. ${ }^{16}$ Glucose metabolism was defined according to the World Health Organization 2006 criteria into normal glucose tolerance (NGT), impaired fasting glucose (IFG), impaired glucose tolerance (IGT), and type 2 diabetes. ${ }^{17}$ Additionally, individuals without type 1 diabetes and on diabetes medication were considered as having type 2 diabetes. ${ }^{16}$ For this study we defined having either IFG or IGT as impaired glucose metabolism (IGM).

\section{Ambulatory blood pressure measurements}

Ambulatory blood pressure was measured with ambulatory 24-hour BP monitoring (WatchBP O3, Microlife AG, Switzerland). Cuffs were applied to the participants' nondominant arm. Measurements were programmed for every 15 minutes during daytime (08.00AM-11.00PM) and every 30 minutes during the night (11.00PM08.00AM), for a total of 24 hours. As quality criteria, mean 24-hour blood pressure measurements were only calculated if there were more than 14 valid measurements at daytime and more than 7 valid measurements at night, based on recommendations of the British Hypertension Society. ${ }^{18,19}$ Mean 24-hour systolic blood pressure (SBP) and diastolic blood pressure (DBP) were calculated based on hourly averages. ${ }^{20}$ 
Twenty-four hour pulse pressure (PP) was defined as 24-hour SBP minus 24-hour DBP and mean arterial pressure (MAP) as 24-hour DBP $+\left(0.412 \times 24\right.$-hour PP). ${ }^{21}$ Mean daytime and night-time SBP and DBP were calculated using measurements between 09.00AM and 09.00PM (more than 14 valid measurements were required) and between 01.00AM and 06.00AM (more than 7 valid measurements were required), respectively. ${ }^{18}$ The relative difference between mean daytime and mean nighttime blood pressure levels was computed and expressed as a percentage of the daytime blood pressure average. Participants were classified as non-dippers when the nocturnal drop in SBP or DBP was less than $10 \%$ of the mean daytime level and as extreme dippers when the nocturnal drop was equal to or larger than $20 \%{ }^{22}$ In all other cases participants were assigned to the dipper category.

\section{Assessment of cognitive function}

A concise battery ( $30 \mathrm{~min})$ of cognitive tests was used to assess cognitive functioning. ${ }^{16}$ An a priori selection of these cognitive tests was used in the current study. Global cognitive functioning was measured by the Mini-Mental State Examination (MMSE). ${ }^{23}$ Verbal memory was assessed with the Visual Verbal Word Learning Test. ${ }^{24}$ In this test, 15 words are presented in five subsequent trials, followed by a recall phase immediately after each trial (immediate recall), and a delayed recall phase 20 minutes thereafter (delayed recall). Response inhibition was measured with the Stroop Color Word Test. ${ }^{25,26}$ The variable of interest was the interference measure expressed in seconds. The Letter-Digit Substitution Test ${ }^{27}$ was used to measure information processing speed. Participants were instructed to match digits to letters as quickly as possible within 90 seconds.

\section{Covariates}

History of cardiovascular disease, diabetes duration, smoking status (never, former, current) and alcohol consumption were assessed by questionnaire. ${ }^{16}$ Participants were regarded as having a history of cardiovascular disease if they reported to have had a: myocardial infarction, and/or cerebrovascular infarction or hemorrhage, and/or percutaneous artery angioplasty of, or vascular surgery on, the coronary, abdominal, peripheral or carotid arteries. Alcohol consumption was classified into three categories: non-consumers, low-consumers ( $\leq 7$ glasses per week for females and $\leq 14$ glasses per week for males) and high-consumers ( $>7$ glasses per week for females and $>14$ glasses per week for males. Lipid-modifying, antihypertensive, and glucoselowering medication use were assessed during a medication interview where generic name, dose and frequency were registered. ${ }^{16}$ Waist circumference, glycosylated hemoglobin A1c (HbA1c), total and high-density lipoprotein (HDL) cholesterol, creatinine, and triglycerides were determined as described elsewhere. ${ }^{16}$ Estimated glomerular filtration rate (eGFR) was estimated using the CKD-EPI (Chronic Kidney Disease Epidemiology Collaboration) equation based on serum creatinine. ${ }^{28}$ Level of 
education was assessed during the cognitive assessment and was classified into 8 categories commonly used in the Netherlands. ${ }^{29}$ (1) no education, (2) primary education, (3) lower vocational education, (4) intermediate general secondary education, (5) intermediate vocational education, (6) higher general secondary education, (7) higher vocational education, (8) university. For this study, three groups were made for educational level: low (level 1 to 3), intermediate (level 4 to 6), and high (level 7-8). Presence of depression was assessed by the Mini International Neuropsychiatric Interview. ${ }^{16,30}$

\section{Statistical analysis}

Analyses were conducted using SPSS version 20 for Mac OSX (SPSS Inc.). Differences between group characteristics were tested using independent samples t-test for continuous variables and $\chi^{2}$-tests for categorical variables. Multiple linear regression analysis was used to estimate the association between ambulatory blood pressure and cognitive performance, stratified by diabetes status. Both linear and quadratic (squared) 24-hour blood pressure terms were included in the models. To control for multicollinearity, linear terms were centered around the mean before forming the quadratic term by subtracting the mean value of the sample from the raw values. When the quadratic term was not significantly associated with any cognitive measure, it was dropped from the models. For analyses with dipping status, three groups were made: 'dippers' (reference group), 'non-dippers', and 'extreme dippers' (see above). Associations were first adjusted for age, sex, and educational level (Model 1), and then for smoking, alcohol, waist circumference, total cholesterol/HDL cholesterol ratio, triglyceride level, antihypertensive medication use, lipid-modifying medication use, eGFR, cardiovascular disease, and depression (Model 2). Response inhibition scores were log-transformed before regression analysis, because they were positively skewed. A two-sided $P$-value of $<0.05$ was considered statistically significant.

\section{Results}

Of the 866 participants included in The Maastricht Study, four individuals with type 1 diabetes and four participants who did not have a cognitive assessment were excluded. Of the remaining 858 participants, we additionally excluded individuals with missing data on the independent variables, i.e. 24-hour blood pressure $(n=88)$ and dipping parameters $(n=116)$, or with missing data on the potential confounders $(n=57)$. This resulted in 713 participants (512 without and 201 with diabetes) for analyses with $24 \mathrm{~h}$ blood pressure and 686 participants (490 without and 196 with diabetes) for dipping variables. Participants who were excluded due to missing values were more often low educated, more likely to be depressed, to be current smokers, 
and to use glucose-lowering medication (particularly insulin). There were no differences in other baseline characteristics (data not shown).

Table 4.1 shows the general characteristics of the 713 individuals included stratified by diabetes status. Of these, 201 participants (28.2\%) had type 2 diabetes, of whom 32 (15.9\%) were newly diagnosed at study entry, $119(59.2 \%)$ used oral medication (of whom 2 participants also used glucagon-like peptide-1 receptor agonists) and 37 (18.4\%) used insulin (of whom 27 also used oral medication). Of the 512 participants without diabetes, 118 participants (16.5\% of the total sample) had IGM. There were no significant differences in performance on any cognitive measure between participants with IGM and those with NGT after adjustment for age and sex ( $P>0.10$ for all measures) (data not shown).

\section{Twenty-four hour SBP, DBP, and cognitive performance}

Table 4.2 shows adjusted linear and quadratic associations between 24-hour blood pressure and cognitive measures. In participants without diabetes, no significant linear or quadratic associations between 24-hour SBP or DBP and cognitive measures were observed. In Models 1 and 2, both 24-hour SBP and DBP were not significantly associated with cognitive performance (Table 4.2). In participants with type 2 diabetes, no linear or quadratic associations were found between 24-hour SBP and cognitive performance after adjustment for potential confounders (Models 1 and 2). However, 24-hour DBP showed quadratic associations with information processing speed, immediate and delayed recall, and response inhibition in Model 1 (Table 4.2). After full adjustment, these associations remained significant, except for response inhibition $(P=0.074)$. These associations indicated that individuals with diabetes and either a low or high 24-hour DBP performed more poorly on tasks for speed, as well as for immediate and delayed word recall than those with a mid-range DBP (an inverted U-shaped relation). Additional analyses showed that associations of 24-hour SBP and DBP with cognitive performance were similar in participants with IGM and NGT (data not shown).

\section{Twenty-four hour PP, MAP and cognitive performance}

In participants without diabetes, neither 24-hour PP nor 24-hour MAP was significantly associated with cognitive performance (Supplementary Table S4.1). In participants with diabetes, we found no significant associations between 24-hour PP and cognitive measures, but the quadratic term of 24-hour MAP was significantly associated with information processing speed (Model 1: $b$ for quadratic term $=-0.0159$, $P=0.004$, Model 2: $b$ for quadratic term $b=-0.0126, P=0.026)$. The quadratic association of 24-hour MAP with delayed word recall was significant in Model 1 (b for quadratic term=-0.0039, $P=0.040$ ), but became non-significant after full adjustment (b for quadratic term $=-0.0035, P=0.069)$. The quadratic association with immediate word recall was not significant (Model 1: $b$ for quadratic term $=-0.0108, P=0.077$; Model 2: $b$ 
for quadratic term $=-0.0064, P=0.294)$. Since another calculation of MAP (24-hour DBP $+1 / 3 *(24$-hour SBP- 24-hour DBP)) has been used previously in other studies, we performed additional analyses with this definition of MAP. Results showed that the quadratic association of 24-hour MAP with delayed word recall increased slightly and became significant in participants with diabetes (Model 2: b for quadratic term $=-0.0042, P=0.042$ ). Other associations remained largely unchanged (data not shown).

Table 4.1 Baseline characteristics of the $\mathbf{7 1 3}$ participants stratified by diabetes status

\begin{tabular}{|c|c|c|c|}
\hline Characteristic & No T2D $(n=512)$ & $T 2 D(n=201)$ & $P$-value ${ }^{a}$ \\
\hline Age, mean (SD) & $58.3(8.5)$ & $63.7(7.0)$ & $<0.001$ \\
\hline Male sex, n (\%) & $248(48.4)$ & $145(72.1)$ & $<0.001$ \\
\hline \multirow[t]{2}{*}{ Educational level, low/middle/high, n (\%) } & $57 / 201 / 254$ & $52 / 102 / 47$ & $<0.001$ \\
\hline & $(11.1 / 39.3 / 49.6)$ & $(25.9 / 50.7 / 23.4)$ & \\
\hline \multirow[t]{2}{*}{ Smoking status, never/former/current, n (\%) } & $180 / 257 / 75$ & $43 / 132 / 26$ & 0.001 \\
\hline & $(35.2 / 50.2 / 14.6)$ & $(21.4 / 65.7 / 12.9)$ & \\
\hline \multirow[t]{2}{*}{ Alcohol consumption, non/low/high, n (\%) } & $65 / 275 / 172$ & $57 / 103 / 41$ & $<0.001$ \\
\hline & $(12.7 / 53.7 / 33.6)$ & $(28.4 / 51.2 / 20.4)$ & \\
\hline Waist circumference (cm), mean (SD) & $93.6(11.8)$ & $106.0(13.0)$ & $<0.001$ \\
\hline Total cholesterol/HDL cholesterol ratio, mean (SD) & $4.24(1.29)$ & $4.15(1.10)$ & 0.34 \\
\hline Triglycerides (mmol/l), median (IQR) & $1.13(0.79-1.58)$ & $1.63(1.12-2.27)$ & $<0.001^{\text {b }}$ \\
\hline Antihypertensive medication, $\mathrm{n}(\%)$ & $141(27.6)$ & $137(68.5)$ & $<0.001$ \\
\hline Lipid-modifying medication, $\mathrm{n}(\%)$ & 100 (19.5) & $157(78.1)$ & $<0.001$ \\
\hline HbA1c (\%), mean (SD) & $5.7(0.4)$ & $6.9(0.8)$ & $<0.001$ \\
\hline $\mathrm{HbA1c}(\mathrm{mmol} / \mathrm{mol})$, mean (SD) & $38.3(4.0)$ & $51.8(8.8)$ & $<0.001$ \\
\hline \multirow[t]{2}{*}{ Glucose-lowering medication use, none/oral/insulin, n (\%) } & - & $45 / 119 / 37$ & \\
\hline & & $(22.5 / 59.2 / 18.4)$ & \\
\hline Diabetes duration (years), median (IQR) & - & $7(3.0-11.0)$ & \\
\hline eGFR $\mathrm{ml} / \mathrm{min} / 1.73 \mathrm{~m}^{2}$, mean $(\mathrm{SD})$ & $85.7(14.1)$ & $82.5(15.0)$ & 0.01 \\
\hline Cardiovascular disease, $\mathrm{n}(\%)$ & $63(12.3)$ & $61(30.3)$ & $<0.001$ \\
\hline Depression, $\mathrm{n}(\%)$ & $15(2.9)$ & $11(5.5)$ & 0.10 \\
\hline SBP 24-hour (mmHg), mean (SD) & $117.7(11.9)$ & $123.7(12.8)$ & $<0.001$ \\
\hline DBP 24-hour (mmHg), mean (SD) & $74.2(7.3)$ & $73.9(7.2)$ & 0.58 \\
\hline PP 24-hour (mmHg), mean (SD) & $43.5(8.0)$ & $49.8(10.3)$ & $<0.001$ \\
\hline MAP 24-hour (mmHg), mean (SD) & $92.2(8.6)$ & $94.4(8.5)$ & 0.002 \\
\hline Dipper status SBP, dippers/non-dippers/ & $258 / 174 / 58$ & $91 / 89 / 16$ & 0.04 \\
\hline extreme dippers, n (\%) & $(52.7 / 35.5 / 11.8)$ & $(46.4 / 45.4 / 8.2)$ & \\
\hline Dipper status DBP, dippers/non-dippers/ & $212 / 56 / 222$ & $72 / 52 / 72$ & $<0.001$ \\
\hline extreme dippers, $\mathrm{n}(\%)$ & $(43.3 / 11.4 / 45.3)$ & $(36.7 / 26.5 / 36.7)$ & \\
\hline Global cognitive functioning (score), mean (SD) & $29.1(1.1)$ & $28.5(1.4)$ & $<0.001$ \\
\hline Information processing speed (number of digits), mean (SD) & $50.4(9.1)$ & $44.2(8.8)$ & $<0.001$ \\
\hline Immediate word recall (number of words), mean (SD) & $47.2(9.2)$ & $40.7(9.4)$ & $<0.001$ \\
\hline Delayed word recall (number of words), mean (SD) & $10.0(2.8)$ & $8.4(2.9)$ & $<0.001$ \\
\hline Response inhibition (seconds), median (IQR) & $39.2(30.8-49.7)$ & $52.2(38.8-70.3)$ & $<0.001^{b}$ \\
\hline
\end{tabular}

${ }^{\mathrm{a}} P$ for difference between groups; ${ }^{\mathrm{b}} P$-values are derived from independent samples T-tests with logtransformed outcomes. For global cognitive functioning, information processing speed, immediate word recall and delayed word recall higher scores indicate better performance. For response inhibition lower scores indicate better performance. T2D, type 2 diabetes; HbA1c, glycosylated hemoglobin A1c; eGFR, estimated glomerular filtration rate; SBP, systolic blood pressure; DBP, diastolic blood pressure; PP, pulse pressure; MAP, mean arterial pressure. 


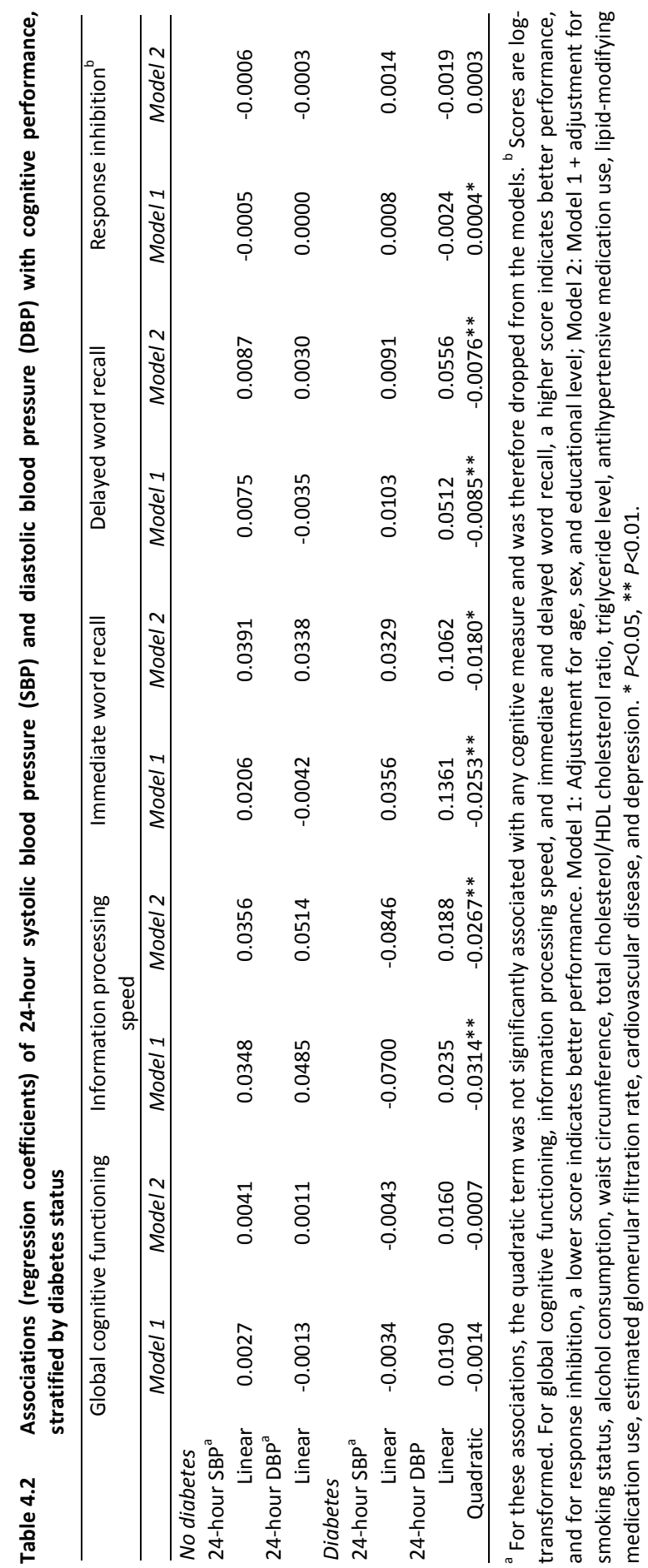




\section{Dipping and cognitive performance}

Supplementary Table S4.2 shows the results of the associations between dipping status and cognitive performance stratified by diabetes status. In participants without diabetes, non-dipping in DBP was associated with slower information processing speed after full adjustment for potential confounders (Model 2: $b=-2.713, P=0.029$ ). Other dipping parameters were not associated with cognitive performance (Supplementary Table S4.2). In participants with diabetes, extreme dipping in SBP was associated with worse global cognitive functioning (Model 2: $b=-0.973, P=0.012$ ). Extreme dipping in DBP was associated with a decreased delayed word recall in participants with diabetes, but only after full adjustment for confounders (Model 2: $b=-0.928, P=0.038)$.

\section{Post-hoc analyses}

Our results showed clear quadratic associations of 24-hour DBP with multiple cognitive tests (information processing speed, and immediate and delayed word recall) in participants with diabetes. These significant associations did not change after further adjustment for the diabetes-related variables $\mathrm{HbA1c}$ level, diabetes duration (available for 168 participants with diabetes), and glucose-lowering medication use (yes/no) (data not shown). Furthermore, these quadratic associations were significantly different in participants with diabetes compared with those without, for information processing speed ( $P$ for interaction between diabetes and quadratic 24 hour DBP=0.001), immediate word recall ( $P$ for interaction $=0.021)$, and delayed word recall ( $P$ for interaction $=0.009$ ) in the fully adjusted model (Model 2 ). These interactions are depicted in Figure 4.1, showing that individuals with type 2 diabetes with either low or high levels of 24-hour DBP performed more poorly on tasks for speed, and immediate / delayed word recall than those with a mid-range DBP (an inverted U-shaped relation), while there was no association between DBP and cognitive performance in individuals without diabetes.

\section{Sensitivity analyses}

Z-scores for 24-hour DBP were calculated for the diabetes group. Participants with Z-scores higher than 3 or smaller than -3 were excluded from analyses to examine whether our results were influenced by extreme observations. One participant had a Z-score $>3$ but exclusion of this participant did not change the results (data not shown). 
A

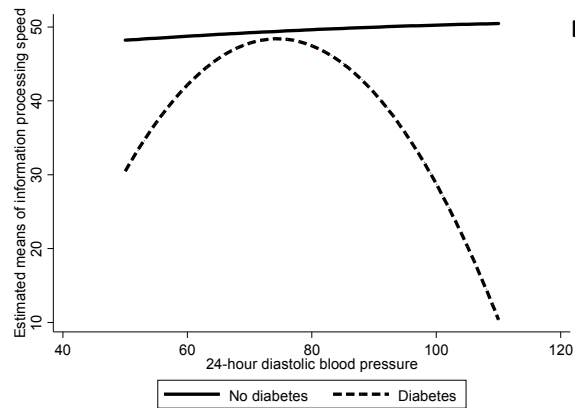

C

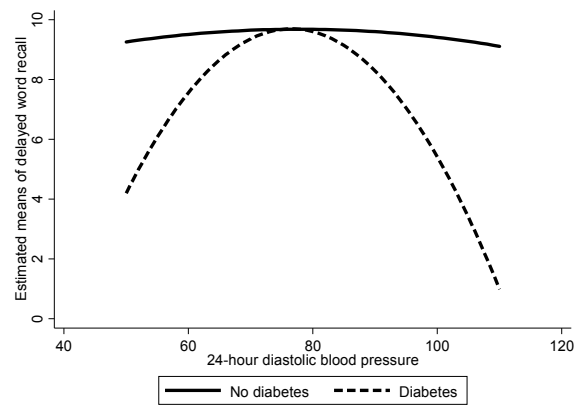

B

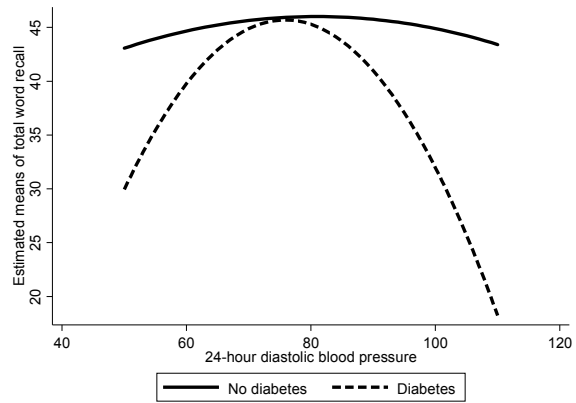

Figure 4.1 Interaction of nonlinear (quadratic) 24-hour diastolic blood pressure $(\mathrm{mmHg})$ and diabetes (solid= no diabetes; dash=diabetes) for: $\mathbf{A}$ : information processing speed (number of digits); B: immediate word recall (number of words); C: delayed word recall (number of words).

\section{Discussion}

In this study individuals with type 2 diabetes with either a low or high 24-hour DBP performed worse on cognitive tests of information processing speed and verbal memory compared with those with a mid-range 24-hour DBP (inverted U-shaped relationship), whereas these associations were not found in individuals without diabetes. In contrast, we found no associations between 24-hour SBP and cognitive performance in either stratum. Finally, dipping status was associated with some measures of cognitive performance in both individuals with and without diabetes, but no clear pattern could be found in these associations.

U-shaped relationships between DBP and cognitive function have been shown previously. ${ }^{14,15}$ Moreover, both low and high office DBP and MAP, but not SBP and PP, have been associated with cognitive impairment 20 years later. ${ }^{15}$ Explanations for this $\mathrm{U}$-shaped relationship may include antihypertensive medication use, arterial stiffness, atherosclerosis, and/or cerebral autoregulation.

In individuals untreated for hypertension, a high office DBP has been associated with hippocampal atrophy, whereas in those treated for hypertension, a low DBP has 
been associated with more hippocampal and amygdalar atrophy. ${ }^{31}$ This may indicate that antihypertensive medication use is involved in the association between DBP and cognitive performance. However, in our study, the U-shaped associations in the diabetes group were attenuated only slightly and remained significant when antihypertensive medication use was added separately to the model in addition to age, sex, and educational level (data not shown).

Another explanation may involve arterial stiffening, which is increased in individuals with type 2 diabetes, $^{32}$ decreases DBP $^{33}$ and, together with atherosclerosis, $^{34}$ may cause hypoperfusion of the brain resulting in a decline in cognitive functions. However, in our study, higher 24-hour PP was not related to information processing speed or memory, arguing against the possibility that arterial stiffening (which typically increases PP) explains our results.

Finally, cerebral autoregulation may be involved in the U-shaped relationship between 24-hour DBP and cognitive performance. Under normal conditions, cerebral blood flow is kept constant across a wide range of MAP $(60-150 \mathrm{mmHg})$ by cerebral autoregulation, which means that arterioles in the brain constrict when SBP rises and dilate when blood pressure falls. ${ }^{35,36}$ However, in individuals with type 2 diabetes the ability to dilate blood vessels may be decreased or even lost, ${ }^{37}$ which may be attributable to cerebral small vessel disease ${ }^{37}$ or cardiovascular autonomic neuropathy. ${ }^{38}$ When autoregulation is lost, cerebral blood flow becomes dependent on the MAP in a linear fashion ${ }^{39}$ and thus decline in blood pressure can lead to hypoperfusion of the brain and consequently cognitive decline. Our finding that a low 24-hour MAP was associated with decreased cognitive performance, particularly information processing speed, in individuals with diabetes fits well with this hypothesis, although this has to be confirmed by future (longitudinal) studies. In addition, orthostatic hypotension, which has been associated with diabetes, ${ }^{40}$ may have an impact on cognitive function, ${ }^{41}$ possibly by recurrent drops in (cerebral) blood pressure.

In the current study, a high DBP was also associated with poorer cognitive performance in participants with diabetes. Previous research has already shown that in individuals with type 2 diabetes, a higher DBP, but not SBP, is associated with cognitive decline, ${ }^{42}$ and that cognitive impairment is associated with cerebral small vessel disease. ${ }^{43}$ Furthermore, high DBP has shown to be an independent predictor of white matter hyperintensity progression ${ }^{44}$ and hippocampal atrophy. ${ }^{31}$ In view of these considerations, it may be hypothesized that a high DBP leads to cognitive impairment through the development of cerebral small vessel disease. Individuals with type 2 diabetes may then be particularly susceptible to the effects of high DBP as they are already at risk to develop cerebral small vessel disease and cognitive impairment.

We could not detect a clear pattern in the associations of non-dipping and extreme dipping with aspects of cognitive performance in participants with and without diabetes. Previous studies, although not stratified by diabetes status, have 
shown inconsistent results with regard to the association of dipping parameters with cognitive performance and cerebrovascular damage. ${ }^{11,45,46}$ These inconsistencies may be due to differences in age, hypertensive status, and duration of hypertension and treatment history of which reliable information can be difficult to obtain. ${ }^{45}$ Stronger associations may be found in older individuals with a long history of hypertension, and especially in older individuals with diabetes, who may have an increased risk of developing cognitive impairment. However, we acknowledge that our sample sizes of the non-dipping and extreme dipping groups were quite small, which limits the power to find significant associations. Finally, it has been suggested that repeated ABPM may increase the accuracy of dipper status assessment ${ }^{47}$ and may be a better predictor of cognitive performance.

To the best of our knowledge this is the first study to examine both linear and nonlinear (quadratic) associations between ABPM and multiple cognitive tests stratified by diabetes status. In one previous study, ${ }^{48}$ the (inverse) association between 24-hour DBP and cognitive performance was stratified by diabetes status in a sub-analysis (stratification did not seem to alter the relationship). However, this study was only performed in older men, lacked data on memory performance, and associations of non-dipping with cognitive functions were not stratified by diabetes status. Another strength of our study is that we were able to adjust for a wide range of potential confounders. Our study is not without limitations. Due to the crosssectional design, we were unable to examine temporality of effects, let alone causal relationships. In addition, we did not have brain imaging data to examine potential mechanisms, e.g. cerebral small vessel disease, which may be involved in the association between DBP and cognitive decline.

In conclusion, this study shows that both low and high 24-hour DBP are associated with poorer performance on tests for information processing speed and memory in individuals with type 2 diabetes, but not in those without diabetes. These results suggest that not only high blood pressure levels, but also low blood pressure levels may increase the risk of cognitive impairment in individuals with type 2 diabetes, which may be important to consider in the therapeutic management of (diastolic) blood pressure levels in those individuals. Monitoring of both blood pressure levels and cognitive performance seems important in individuals with type 2 diabetes. Future (longitudinal) studies should focus on causality and underlying mechanisms of these associations. 


\section{References}

1. Spauwen PJ, Kohler S, Verhey FR, Stehouwer CD, van Boxtel MP. Effects of type 2 diabetes on 12-year cognitive change: results from the maastricht aging study. Diabetes Care 2013;36:1554-61

2. Biessels GJ, Staekenborg S, Brunner E, Brayne C, Scheltens P. Risk of dementia in diabetes mellitus: a systematic review. Lancet Neurol 2006;5:64-74

3. van den Berg E, Dekker JM, Nijpels G, et al. Blood pressure levels in pre-diabetic stages are associated with worse cognitive functioning in patients with type 2 diabetes. Diabetes Metab Res Rev 2009;25:657-64

4. Bruce DG, Davis WA, Casey GP, et al. Predictors of cognitive impairment and dementia in older people with diabetes. Diabetologia 2008;51:241-8

5. Hassing LB, Hofer SM, Nilsson SE, et al. Comorbid type 2 diabetes mellitus and hypertension exacerbates cognitive decline: evidence from a longitudinal study. Age Ageing 2004;33:355-61

6. Xu WL, Qiu CX, Wahlin A, Winblad B, Fratiglioni L. Diabetes mellitus and risk of dementia in the Kungsholmen project: a 6-year follow-up study. Neurology 2004;63:1181-6

7. Korf ES, van Straaten EC, de Leeuw FE, et al. Diabetes mellitus, hypertension and medial temporal lobe atrophy: the LADIS study. Diabet Med 2007;24:166-71

8. Gregg EW, Yaffe K, Cauley JA, et al. Is diabetes associated with cognitive impairment and cognitive decline among older women? Study of Osteoporotic Fractures Research Group. Arch Intern Med 2000;160:174-80

9. White WB, Wolfson L, Wakefield DB, et al. Average daily blood pressure, not office blood pressure, is associated with progression of cerebrovascular disease and cognitive decline in older people. Circulation 2011;124:2312-9

10. Yano Y, Kario K. Nocturnal blood pressure, morning blood pressure surge, and cerebrovascular events. Curr Hypertens Rep 2012;14:219-27

11. van Boxtel MP, Gaillard C, Houx PJ, Buntinx F, de Leeuw PW, Jolles J. Is nondipping in $24 \mathrm{~h}$ ambulatory blood pressure related to cognitive dysfunction? J Hypertens 1998;16:1425-32

12. Okuno J, Yanagi H. Cognitive impairment and nocturnal blood pressure fall in treated elderly hypertensives. Environ Health Prev Med 2003;8:124-32

13. Ayala DE, Moya A, Crespo JJ, et al. Circadian pattern of ambulatory blood pressure in hypertensive patients with and without type 2 diabetes. Chronobiol Int 2013;30:99-115

14. Waldstein SR, Giggey PP, Thayer JF, Zonderman AB. Nonlinear relations of blood pressure to cognitive function: the Baltimore Longitudinal Study of Aging. Hypertension 2005;45:374-9

15. Taylor C, Tillin T, Chaturvedi N, et al. Midlife hypertensive status and cognitive function 20 years later: the Southall and Brent revisited study. J Am Geriatr Soc 2013;61:1489-98

16. Schram MT, Sep SJ, van der Kallen CJ, et al. The Maastricht Study: an extensive phenotyping study on determinants of type 2 diabetes, its complications and its comorbidities. Eur J Epidemiol 2014;29: 439-51

17. World Health Organization. Definition and Diagnosis of Diabetes Mellitus and Intermediate Hyperglycemia: Report of a WHO/IDF Consultation. Geneva, World Health Organization, 2006

18. O'Brien $E$, Coats $A$, Owens $P$, et al. Use and interpretation of ambulatory blood pressure monitoring: recommendations of the British hypertension society. BMJ 2000;320:1128-34

19. Muris DM, Houben AJ, Kroon AA, et al. Age, waist circumference, and blood pressure are associated with skin microvascular flow motion: The Maastricht Study. J Hypertens 2014;32:2439-49; discussion 2449

20. Mancia G, Fagard R, Narkiewicz K, et al. 2013 ESH/ESC guidelines for the management of arterial hypertension: the Task Force for the Management of Arterial Hypertension of the European Society of Hypertension (ESH) and of the European Society of Cardiology (ESC). Eur Heart J 2013;34:2159-219

21. Meaney E, Alva F, Moguel R, Meaney A, Alva J, Webel R. Formula and nomogram for the sphygmomanometric calculation of the mean arterial pressure. Heart 2000;84:64

22. Kario K, Shimada K, Pickering TG. Abnormal nocturnal blood pressure falls in elderly hypertension: clinical significance and determinants. J Cardiovasc Pharmacol 2003;41 Suppl 1:S61-6

23. Folstein MF, Folstein SE, McHugh PR. "Mini-mental state". A practical method for grading the cognitive state of patients for the clinician. J Psychiatr Res 1975;12:189-98 
24. Van der Elst W, van Boxtel MP, van Breukelen GJ, Jolles J. Rey's verbal learning test: normative data for 1855 healthy participants aged 24-81 years and the influence of age, sex, education, and mode of presentation. J Int Neuropsychol Soc 2005;11:290-302

25. Stroop J. Studies of interference in serial verbal reaction. J Exp Psychol 1935;18:643-62

26. Van der Elst W, Van Boxtel MP, Van Breukelen GJ, Jolles J. The Stroop color-word test: influence of age, sex, and education; and normative data for a large sample across the adult age range. Assessment 2006;13:62-79

27. van der Elst W, van Boxtel MP, van Breukelen GJ, Jolles J. The Letter Digit Substitution Test: normative data for 1,858 healthy participants aged 24-81 from the Maastricht Aging Study (MAAS): influence of age, education, and sex. J Clin Exp Neuropsychol 2006;28:998-1009

28. Levey AS, Stevens LA, Schmid $\mathrm{CH}$, et al. A new equation to estimate glomerular filtration rate. Ann Intern Med 2009;150:604-12

29. De Bie SE. Standaardvragen 1987: Voorstellen voor uniformering van vraagstellingen naar achtergrondkenmerken en interviews. Leiden, Leiden University Press, 1987

30. Sheehan DV, Lecrubier $\mathrm{Y}$, Sheehan $\mathrm{KH}$, et al. The Mini-International Neuropsychiatric Interview (M.I.N.I.): the development and validation of a structured diagnostic psychiatric interview for DSM-IV and ICD-10. J Clin Psychiatry 1998;59 Suppl 20:22-33;quiz 4-57

31. den Heijer T, Launer LJ, Prins ND, et al. Association between blood pressure, white matter lesions, and atrophy of the medial temporal lobe. Neurology 2005;64:263-7

32. Stehouwer CD, Henry RM, Ferreira I. Arterial stiffness in diabetes and the metabolic syndrome: a pathway to cardiovascular disease. Diabetologia 2008;51:527-39

33. Witteman JC, Grobbee DE, Valkenburg HA, et al. J-shaped relation between change in diastolic blood pressure and progression of aortic atherosclerosis. Lancet 1994;343:504-7

34. Creager MA, Luscher TF, Cosentino F, Beckman JA. Diabetes and vascular disease: pathophysiology, clinical consequences, and medical therapy: Part I. Circulation 2003;108:1527-32

35. Lassen NA. Cerebral blood flow and oxygen consumption in man. Physiol Rev 1959;39:183-238

36. Paulson OB, Strandgaard S, Edvinsson L. Cerebral autoregulation. Cerebrovasc Brain Metab Rev 1990;2:161-92

37. Kim YS, Immink RV, Stok WJ, Karemaker JM, Secher NH, van Lieshout JJ. Dynamic cerebral autoregulatory capacity is affected early in Type 2 diabetes. Clin Sci (Lond) 2008;115:255-62

38. Marthol $\mathrm{H}$, Brown $\mathrm{CM}$, Zikeli $\mathrm{U}$, et al. Altered cerebral regulation in type 2 diabetic patients with cardiac autonomic neuropathy. Diabetologia 2006;49:2481-7

39. Cipolla MJ. Control of Cerebral Blood Flow. In: Cerebral Circulation. San Rafael (CA): Morgan \& Claypool Life Science; 2009.

40. Low PA, Benrud-Larson LM, Sletten DM, et al. Autonomic symptoms and diabetic neuropathy: a population-based study. Diabetes Care 2004;27:2942-7

41. Mehrabian S, Duron E, Labouree F, et al. Relationship between orthostatic hypotension and cognitive impairment in the elderly. J Neurol Sci 2010;299:45-8

42. Umegaki $\mathrm{H}$, limuro $\mathrm{S}$, Shinozaki $\mathrm{T}$, et al. Risk factors associated with cognitive decline in the elderly with type 2 diabetes: pooled logistic analysis of a 6-year observation in the Japanese Elderly Diabetes Intervention Trial. Geriatr Gerontol Int 2012;12 Suppl 1:110-6

43. Akisaki T, Sakurai T, Takata T, et al. Cognitive dysfunction associates with white matter hyperintensities and subcortical atrophy on magnetic resonance imaging of the elderly diabetes mellitus Japanese elderly diabetes intervention trial (J-EDIT). Diabetes Metab Res Rev 2006;22:376-84

44. Schmidt R, Schmidt H, Kapeller P, Lechner A, Fazekas F. Evolution of white matter lesions. Cerebrovasc Dis 2002;13 Suppl 2:16-20

45. van Boxtel MP, Henskens LH, Kroon AA, et al. Ambulatory blood pressure, asymptomatic cerebrovascular damage and cognitive function in essential hypertension. I Hum Hypertens 2006;20:5-13

46. Sierra C, de La Sierra A, Mercader J, Gomez-Angelats E, Urbano-Marquez A, Coca A. Silent cerebral white matter lesions in middle-aged essential hypertensive patients. J Hypertens 2002;20:519-24

47. Cuspidi C, Macca G, Michev I, et al. Short-term reproducibility of nocturnal non-dipping pattern in recently diagnosed essential hypertensives. Blood Press 2002;11:79-83

48. Kilander L, Nyman $\mathrm{H}$, Boberg $\mathrm{M}$, Hansson L, Lithell $\mathrm{H}$. Hypertension is related to cognitive impairment: a 20-year follow-up of 999 men. Hypertension 1998;31:780-6 


\section{Supplemental data}

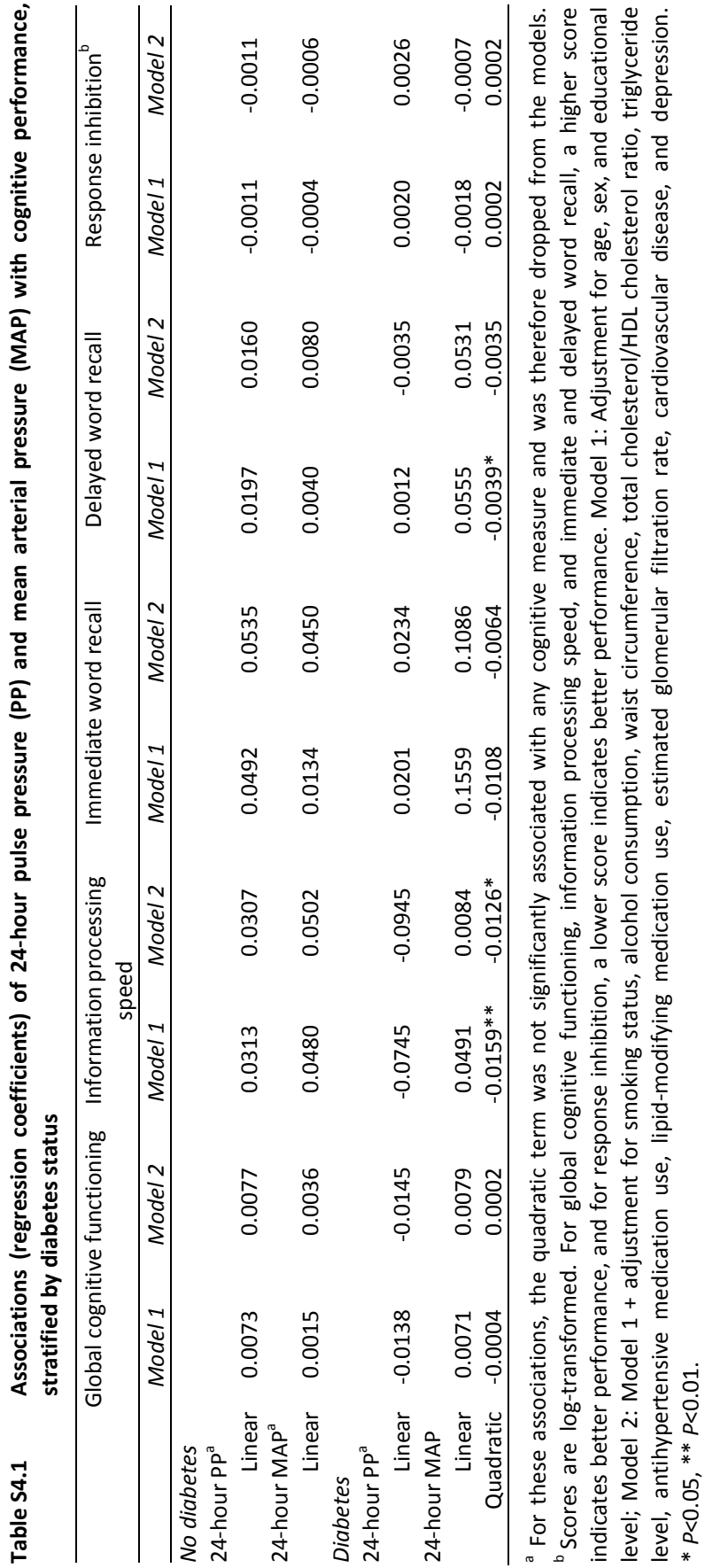




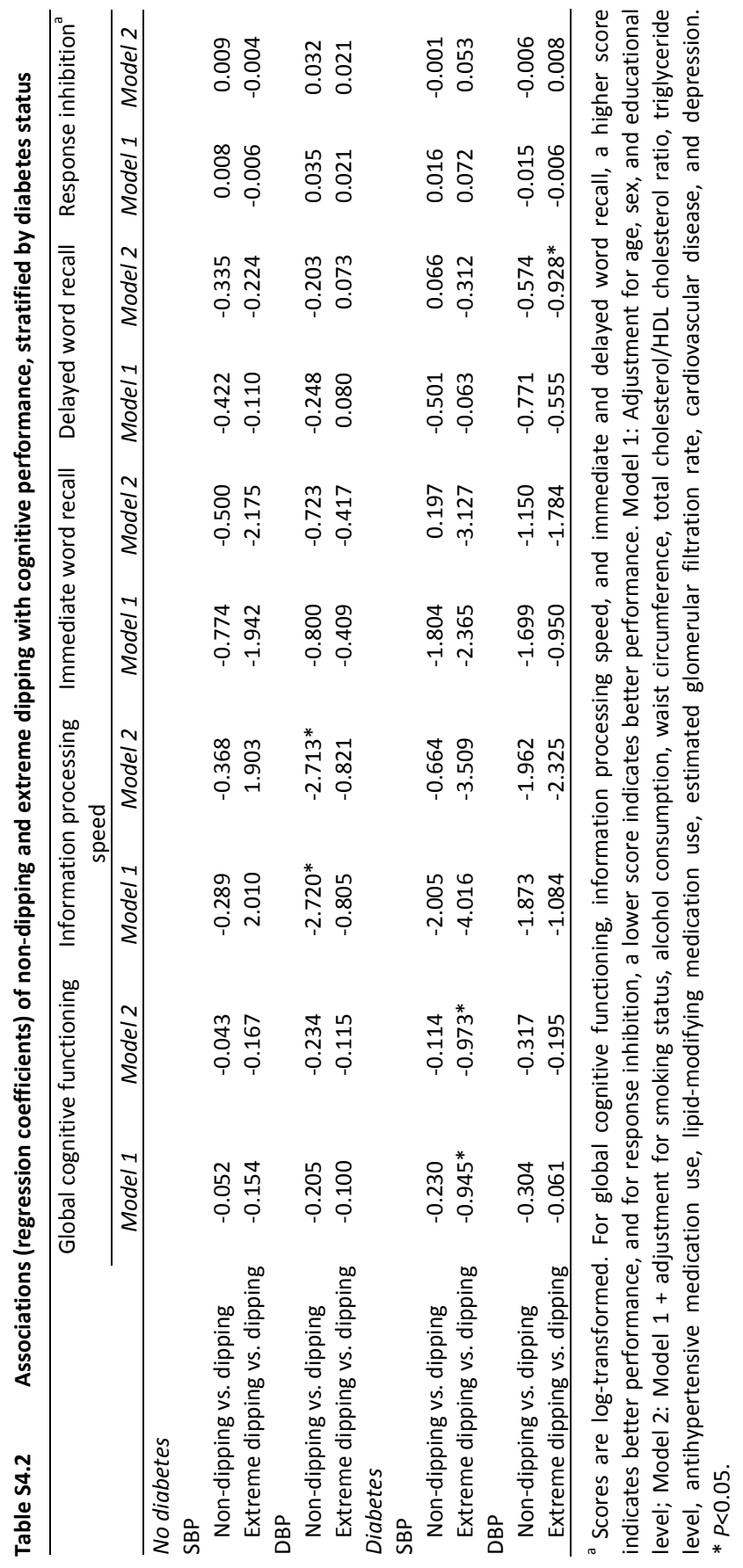




\section{Chapter 5}

\section{ASSOCIATIONS OF FAT AND MUSCLE TISSUE WITH COGNITIVE STATUS IN OLDER ADULTS:}

THE AGES-REYKJAVIK STUDY

PJJ Spauwen, RA Murphy, PV Jonsson, S Sigurdsson, ME Garcia, G Eiriksdottir, MPJ van Boxtel, OL Lopez, V Gudnason, TB Harris, LJ Launer 


\section{Abstract}

\section{Importance}

Studies on the association between specific body composition components and dementia are scarce. Knowing more about specific patterns of body composition associated with cognitive function may help to better understand the underlying mechanisms regulating weight in prodromal and clinical stages of dementia.

\section{Objective}

To investigate associations of body composition measures with different levels of cognitive function in late life.

\section{Design}

Cross-sectional data from the AGES-Reykjavik Study (2002-2006).

\section{Setting}

Population-based cohort.

\section{Participants}

A total of 5,169 participants (mean age 76 years, 42.9\% men) of whom 485 (9.4\%) were diagnosed with Mild Cognitive Impairment (MCl) and 307 (5.9\%) with dementia.

\section{Main outcome measures}

Visceral fat, abdominal and thigh subcutaneous fat, and thigh muscle were assessed by computed tomography. $\mathrm{MCl}$ and dementia were based on clinical assessment and a consensus meeting; those without $\mathrm{MCl}$ or dementia were categorized as normal (reference category). Associations were assessed with multinomial regression analyses stratified by sex, and adjusted for demographics, vascular risk factors, stroke, depression, weight change from midlife, and the Apolipoprotein $\mathrm{E} \varepsilon 4$ allele. In addition, analyses were stratified by midlife body mass index (BMI).

\section{Results}

Among women, there was a decreased likelihood of dementia per SD increase in abdominal subcutaneous fat $(\mathrm{OR}=0.72 ; 95 \% \mathrm{Cl}=0.59$ to 0.88$)$, thigh subcutaneous fat $(0.81 ; 0.67$ to 0.98$)$ and thigh muscle $(0.63 ; 0.52$ to 0.76$)$, but not visceral fat, adjusting for demographics, vascular risk factors, stroke, and depression. The inverse associations of fat with dementia were attenuated by change in weight from midlife and were strongest in women with a midlife $\mathrm{BMI}<25$. In men, one SD increase in thigh muscle was associated with a decreased likelihood of dementia $(0.75 ; 0.61$ to 0.92$)$. There was no association between body composition and $\mathrm{MCl}$ in men or women.

\section{Conclusions and relevance}

A higher amount of subcutaneous fat, located both in the thigh and abdomen, was associated with decreased odds of dementia in women only, while more thigh muscle was associated with decreased dementia odds in men and women. Further studies are needed to explore longitudinally, mechanisms underlying the relationship between changes in body composition and cognitive decline. 


\section{Introduction}

Obesity in midlife has been associated with greater cognitive decline and increased risk of dementia. ${ }^{1,2}$ However, in late life, obesity has been associated with a decreased likelihood of having dementia, ${ }^{3-5}$ possibly due to weight loss in the prodromal phase of dementia. ${ }^{6}$ Most studies examining the relationship between obesity and dementia infer an association of adiposity with dementia, but use indirect measurements, such as BMI and waist circumference. However, aging is associated with an increase in fat mass and a decrease in lean mass, making BMI a less suitable measurement for total body adiposity in older persons. Additionally BMI does not provide a measure of muscle $^{7}$ or of the specific fat components of visceral and subcutaneous fat, which are related to different metabolic profiles. For example, compared to subcutaneous fat, visceral fat has been shown to be more metabolically active and is more strongly associated with insulin resistance, ${ }^{8}$ although subcutaneous fat has also been associated with insulin resistance. ${ }^{9}$

Studies on the association between specific body composition (BC) components and dementia are scarce and based on a small sample. ${ }^{10}$ Knowing more about specific patterns of $\mathrm{BC}$ associated with different levels of cognitive function may help to better understand the underlying mechanisms regulating weight in prodromal and clinical stages of dementia, and to better advise interventions for older persons showing cognitive decline.

Here, we examine in a large well-characterized cohort followed from mid to late life the association of the amount of abdominal fat (visceral and subcutaneous), thigh subcutaneous fat, and thigh lean muscle tissue with Mild Cognitive Impairment $(\mathrm{MCl})$, considered an intermediary stage to dementia, and dementia in older persons. In addition, taking previous studies into account, ${ }^{1,2}$ we investigate whether these associations are different in persons with a low midlife BMI compared with those with a high midlife BMI.

\section{Methods}

\section{Subjects}

The Age Gene/Environment Susceptibility (AGES)-Reykjavik Study has been described in detail. ${ }^{11}$ Briefly, the Reykjavik Study is a population-based cohort study established in 1967 by the Icelandic Heart Association to prospectively study cardiovascular disease in Iceland. The cohort included a random sample of men and women born between 1907 and 1935 and living in Reykjavik at baseline. The study was continued in 2002, as the AGES-Reykjavik Study, with the aim of investigating risk factors, genetic susceptibility, and gene-environment interactions in relation to disease and disability in old age, as well as healthy aging. ${ }^{11}$ Of the 11,549 (58\% women) surviving members 
of the Reykjavik cohort 8,030 ( $68.6 \%$ of men and $70.1 \%$ of women) were invited to participate in the AGES-Reykjavik Study; $71.8 \%$ participated $(74.0 \%$ of men and $70.2 \%$ of women), giving a final sample of 5,764 (58\% women). ${ }^{11}$ Each participant underwent exams that included CT and MRI imaging, cognitive, physical, and clinical measures, as well as the administration of questionnaires that sought information on medical history, and lifestyle factors.

Informed consent was obtained from all participants. The study was approved by the National Bioethics Committee in Iceland, which acts as the Institutional Review Board for the IHA (VSN-00-063), and by the National Institute on Aging Intramural Institutional Review Board.

\section{Measures}

\section{Cognitive status (normal-MCl-dementia)}

The case finding was based on a 3-step procedure, previously described. ${ }^{11}$ Briefly, all participants were screened on the Mini-Mental State Examination (MMSE) and the Digit Symbol Substitution Test (DSST). Screen positives on the MMSE (score of 23 or lower) or the DSST (score of 17 or lower) received another more complete diagnostic battery. Those who were screen positive on Trial Making Test A and B or the Rey Auditory Verbal Learning Test went for a final assessment that included a proxy interview and a neurologic examination. ${ }^{12}$ Dementia and $\mathrm{MCl}$ were diagnosed by a consensus conference that included a geriatrician, a neurologist, a neuropsychologist, and a neuroradiologist following the criteria of the Diagnostic and Statistical Manual of Mental Disorders, Fourth Edition. ${ }^{13} \mathrm{MCl}$ was defined by cognitive performance (scoring <-1.5 SDs below a cut-point determined from the distribution of scores in a subsample of the cohort) on memory or two other domains and the absence of evidence that cognition interfered with daily functioning. ${ }^{14}$

\section{Body composition}

CT imaging of the mid-thigh and abdomen at the L4/L5 vertebrae was performed with a 4-row detector system (Sensation; Siemens Medical Systems, Erlangen, Germany) and has been described previously. ${ }^{15}$ Abdominal visceral fat and subcutaneous fat were estimated from a single $10 \mathrm{~mm}$ thick trans-axial section. Abdominal visceral fat was distinguished from subcutaneous fat by tracing along the facial plane defining the internal abdominal wall. Adipose areas were calculated by multiplying the number of pixels by the pixel area using specialized software (University of California, San Francisco). Thigh subcutaneous fat and muscle were estimated from a single $10 \mathrm{~mm}$ thick axial section as described previously. ${ }^{16}$ Total thigh subcutaneous fat and muscle were determined from the right and left thigh. When data for one leg was missing $(n=14)$, data from the other leg were multiplied by 2 . 
BMI was calculated as measured weight in kilograms divided by measured height in meters squared. Mid-life BMI was categorized as low to normal BMI $\left(<25.0 \mathrm{~kg} / \mathrm{m}^{2}\right)$ and overweight to obese $\left(\geq 25.0 \mathrm{~kg} / \mathrm{m}^{2}\right){ }^{4}$ Percentage weight change from midlife was calculated as: ((late-life weight - midlife weight)/ midlife weight)) x 100 .

\section{Covariates}

In our analyses we corrected for factors that may confound the association between BC and cognitive status: age, education (primary, secondary, or higher than secondary), depressive symptoms assessed with the 15-item Geriatric Depression Scale (GDS) (high depression symptomatology was classified as a score of 6 or greater), ${ }^{17}$ and smoking history (never, former, or current).

We also examined the effect of various factors that might confound as well as mediate an association between $\mathrm{BC}$ and cognitive status: fasting blood samples were used to measure: HDL cholesterol, triglycerides (TG) levels and high-sensitivity C-reactive protein (hs-CRP) on a Hitachi 912, using reagents from Roche Diagnostics and following the manufacturer's instructions. For this analysis, level of TG was divided by level of HDL cholesterol to derive the TG-to-HDL ratio (TG/HDL ratio), an indicator of insulin resistance ${ }^{18}$ and marker for risk of coronary heart disease. ${ }^{19}$ Presence of type 2 diabetes was based on World Health Organization recommendations of a serum glucose $>7 \mathrm{mmol} / \mathrm{I}^{20}{ }^{20}$ self-reported physician's diabetes, or use of diabetes medication. Hypertension was based on self-reported physician's diagnosis, use of hypertensive medication, or systolic blood pressure $\geq 140$ or diastolic blood pressure $\geq 90 \mathrm{mmHg}$. Prevalent stroke was based on adjudicated data from hospital registries as well as self-reported diagnosis of stroke. We also included the presence (Participants with $\varepsilon 2 / 4, \varepsilon 3 / 4$, and $\varepsilon 4 / 4$ genotype) or absence $(\varepsilon 2 / 2, \varepsilon 2 / 3$ and $\varepsilon 3 / 3$ genotype) of an Apolipoprotein E (APOE) $\varepsilon 4$ allele. Genotyped with the microplate array diagonal gel electrophoresis system. ${ }^{21}$

\section{Analytical sample}

Of the 5,764 participants, the 252 with missing data on cognitive status, the additional 296 with missing CT-scan data, and the 47 with missing data on various confounders were excluded from the analysis, giving a final sample of 5,169. Participants excluded due to missing data ( $n=595)$, were significantly older, were more likely to be female, to have less formal education, to have diabetes, to have had a stroke, to report depressive symptoms, less likely to report a history of smoking, had higher hs-CRP levels, a higher TG/HDL ratio, and gained less weight from midlife.

Further, of the 5,169 participants, we coded the 269 participants with missing data for the 15-point GDS score into a third category for missing $(\geq 6$, considered to have depression symptomatology, $<6$ having no significant symptomatology, and missing). 


\section{Statistical analysis}

Because of known differences in BC between men and women, all analyses were stratified by sex. Sex-specific Z-scores were calculated for each individual (individual value for the $B C$ measure minus the mean for their sex and divided by the SD for their sex). Differences in baseline characteristics were analyzed with ANOVA for continuous variables and Chi-square tests for categorical variables.

Multinomial regression techniques were used to analyze the association between continuous Z-score values of abdominal fat, thigh subcutaneous fat, and thigh muscle (independent variables) and cognitive status (normal, $\mathrm{MCl}$, and demented). We adjusted models of adiposity, muscle and cognitive status for age (model 0), plus other potential confounders (Model 1; educational level, smoking status, and depressive symptoms). For Model 2, we added to Model 1, potential mediators (diabetes, hypertension, stroke, TG/HDL ratio, hs-CRP), and in Model 3, we added to Model 2, change in weight from midlife). In addition, we investigated the potential moderating effect of having an APOE $\varepsilon 4$ allele by testing the statistical significance of the interaction between APOE $\varepsilon 4$ and BC measures (APOE $\varepsilon 4$ status * BC variable) on cognitive status in Model 2. As there were generally no significant interactions, we do not report further on this.

Finally, we performed the analyses stratified by midlife BMI category (lownormal: $\mathrm{BMI}<25$; overweight-obese: $\mathrm{BMI} \geq 25$ ) and additionally tested the interaction between midlife BMI category and $\mathrm{BC}$ measures (midlife BMI category* $\mathrm{BC}$ variable) on cognitive status in Model 2.

Analyses were conducted using IBM SPSS Statistics for Macintosh, version 20 (IBM Corp., Armonk, NY, USA). A P-value of 0.05 was considered statistically significant.

\section{Results}

\section{Baseline characteristics}

Of the 5,169 participants, 2,217 were men (42.9\%), of whom $249(11.2 \%)$ had $\mathrm{MCl}$ and 146 (6.6 \%) had dementia (Table 5.1. Mean age of the total sample was 76.4 years $(S D=5.5)$. Of the 2,952 woman (57.1\%), $236(8.0 \%)$ had $\mathrm{MCl}$ and 161 (5.5\%) had dementia. Mean age of midlife weight and BMI assessment was 49.9 years (SD=6.1) for men and 52.2 years $(S D=7.0)$ for women. Further descriptions of the cohort are found in Table 5.1. Men had more visceral fat and muscle tissue than women, while women had more abdominal and thigh subcutaneous fat than men (Table 5.2). 


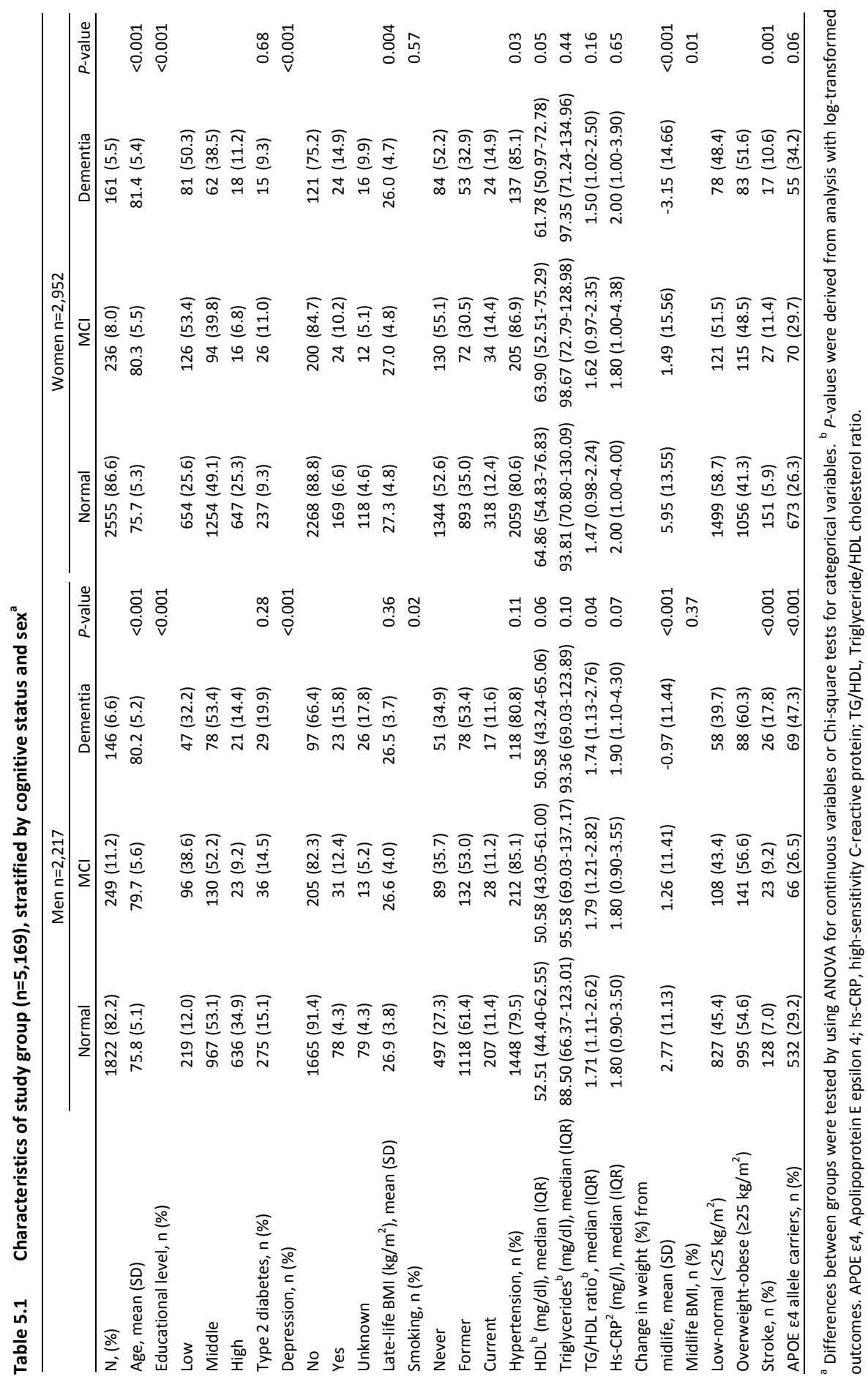


Table 5.2 Body composition measures (mean, (SD)) in men and women

\begin{tabular}{lrc}
\hline & Men $(\mathrm{n}=2,217)$ & Women $(\mathrm{n}=2,952)$ \\
\hline Visceral fat $\left(\mathrm{cm}^{2}\right)$ & $202.79(85.96)$ & $150.20(67.13)$ \\
Abdominal subcutaneous fat $\left(\mathrm{cm}^{2}\right)$ & $203.26(86.84)$ & $295.60(112.37)$ \\
Thigh subcutaneous fat $\left(\mathrm{cm}^{2}\right)$ & $78.02(38.93)$ & $186.93(82.08)$ \\
Thigh muscle $\left(\mathrm{cm}^{2}\right)$ & $254.08(41.58)$ & $184.60(30.27)$ \\
\hline
\end{tabular}

\section{Body composition and cognitive status}

In women, visceral fat was not significantly associated with dementia (Figure 5.1; Supplementary Table S5.1). In contrast, abdominal subcutaneous fat was significantly associated with dementia, such that an increment of one SD in abdominal subcutaneous fat was associated with a $28 \%$ decreased odds of dementia in women (Figure 5.1; Supplementary Table S5.1, Model 2).

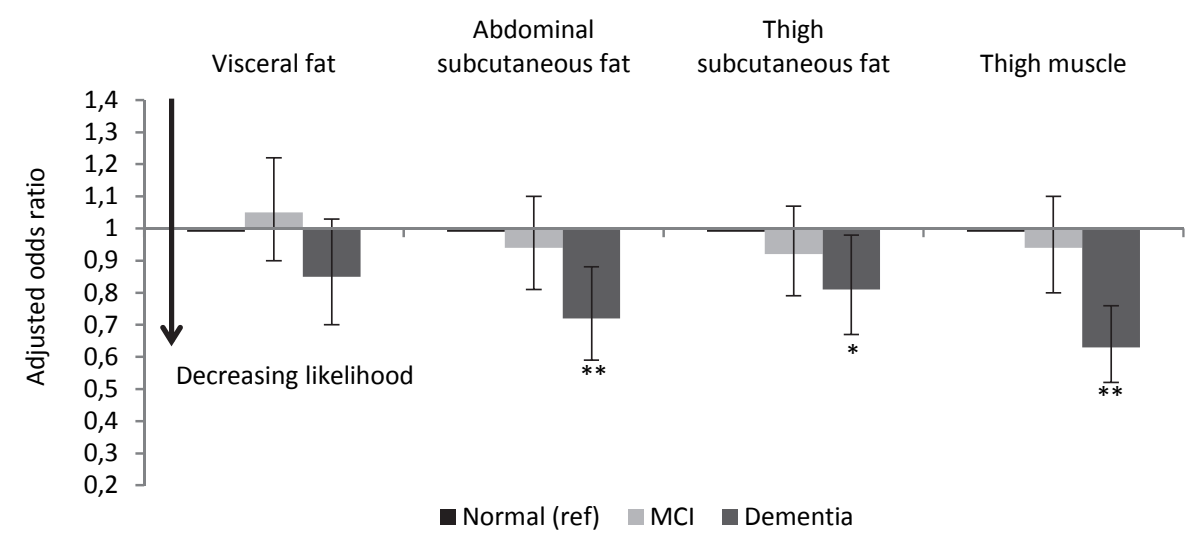

Figure 5.1 Associations between body composition and cognitive status in women.

Bars are odds ratios per SD increase in visceral, abdominal and thigh subcutaneous fat, and muscle tissue, adjusted for age, educational level, smoking status, depressive symptoms, type 2 diabetes, hypertension, stroke, triglyceride/HDL cholesterol ratio, and high-sensitivity C-reactive protein (Model 2), and error bars are $95 \%$ confidence intervals.

$* P$-value $<0.05, * * P$-value $<0.01$.

After further adjustment for weight change from midlife, which was negatively associated with dementia (less weight gain/more weight loss was associated with increased odds of dementia), this association attenuated and became non-significant (Figure 5.2; Supplementary Table S5.1, Model 3). Thigh subcutaneous fat was also significantly associated with decreased odds of dementia in Models 0-2 (Supplementary Table S5.1). One SD increase in subcutaneous fat was associated with a $19 \%$ decreased odds of dementia in women (Figure 5.1; Supplementary Table S5.1, Model 2). This association was attenuated and became non-significant after further 
adjustment for weight change from midlife (Figure 5.2; Supplementary Table S5.1, Model 3). Finally, in women, thigh muscle was significantly associated with decreased odds of dementia, whereby an increment of one SD in thigh muscle was associated with a $37 \%$ decreased odds of dementia (Figure 5.1; Supplementary Table S5.1, Model 2). This association was attenuated slightly after adjustment for weight change from midlife, but remained statistically significant (Figure 5.2; Supplementary Table S5.1, Model 3).

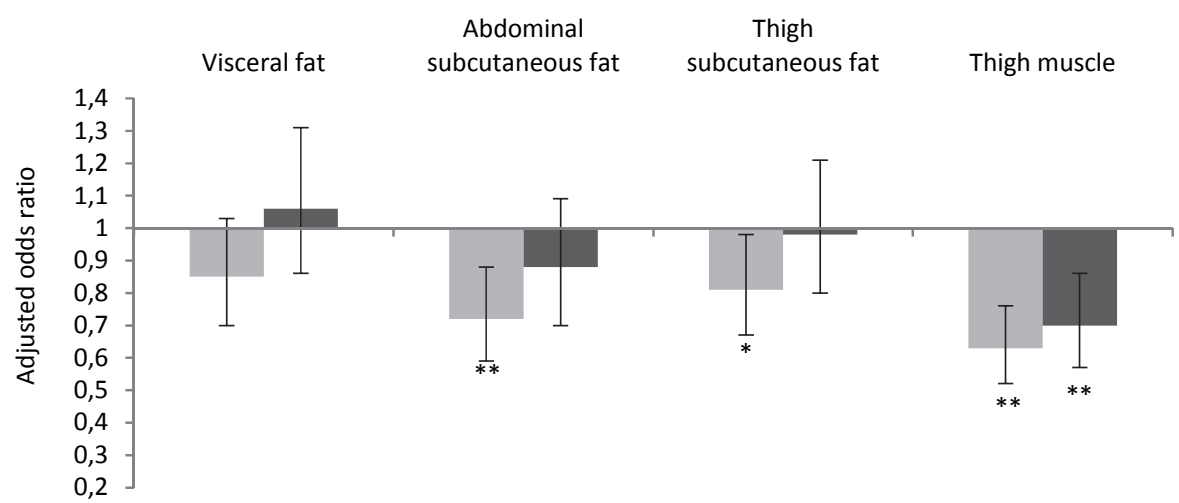

Model 2 Model 3

Figure 5.2 Associations between body composition and cognitive status in women before (Model 2) and after (Model 3) additional adjustment for percentage change in weight from midlife. Bars are odds ratios for dementia per SD increase in visceral, abdominal and thigh subcutaneous fat, and muscle tissue, and error bars are $95 \%$ confidence intervals.

* $P$-value $<0.05, * * P$-value $<0.01$.

In women, $\mathrm{BC}$ measures were not significantly associated with $\mathrm{MCl}$; however the ORs for abdominal, thigh subcutaneous fat, and muscle tended to be intermediate to the ORs of those who were cognitively normal and those with dementia (Figure 5.1; Supplementary Table S5.1, Models 0-2). In Model 3, these associations attenuated somewhat (Supplementary Table S5.1, Model 3).

In men, fat measures were not associated with cognitive status (Supplementary Table S5.1, Models 0-2). In contrast, an increment of one SD in thigh muscle was significantly associated with a $25 \%$ decreased odds of dementia (Supplementary Table S5.1, Model 2). Adjusting for weight change from midlife did not change the association (Supplementary Table S5.1, Model 3). BC measures were not associated with $\mathrm{MCl}$ in men (Supplementary Table S5.1, Models 0-2).

\section{Analyses stratified by BMI at midlife}

In women with a low-normal midlife BMI (midlife BMI <25), the likelihood of dementia significantly decreased per SD increase in visceral fat, abdominal and thigh subcutaneous fat. Associations in the overweight-obese group (midlife BMI $\geq 25$ ) were 
attenuated towards the null for the subcutaneous fat and visceral fat body components (Figure 5.3; Supplementary Table S5.2, Model 2) $\left(P_{\text {interaction visceral fat }}=0.10\right.$; $\left.P_{\text {interaction abdominal subcutaneous fat }}=0.07 ; P_{\text {interaction thigh subcutaneous fat }}=0.03\right)$. Per SD increase in thigh muscle the likelihood of dementia significantly decreased in both women with midlife BMI $<25$ and midlife BMI $\geq 25$ (Figure 5.3; Supplementary Table S5.2, Model 2) $\left(P_{\text {interaction muscle }}=0.19\right)$. There were no significant associations between BC measures and $\mathrm{MCl}$ in women with a $\mathrm{BMI}<25$ or $\mathrm{BMI} \geq 25$ (Supplementary Table S5.2, Models 0-2).

In men, measures of fat were not associated with dementia in either of the midlife BMI strata (Supplementary Table S5.2, Models 0-2). In contrast, an increase of one SD in thigh muscle was associated with decreased odds of dementia in men with a midlife BMI $\geq 25$, but not with a midlife BMI $<25$ (Supplementary Table S5.2, Model 2) $\left(P_{\text {interaction muscle }}=0.03\right.$, Model 2$)$.

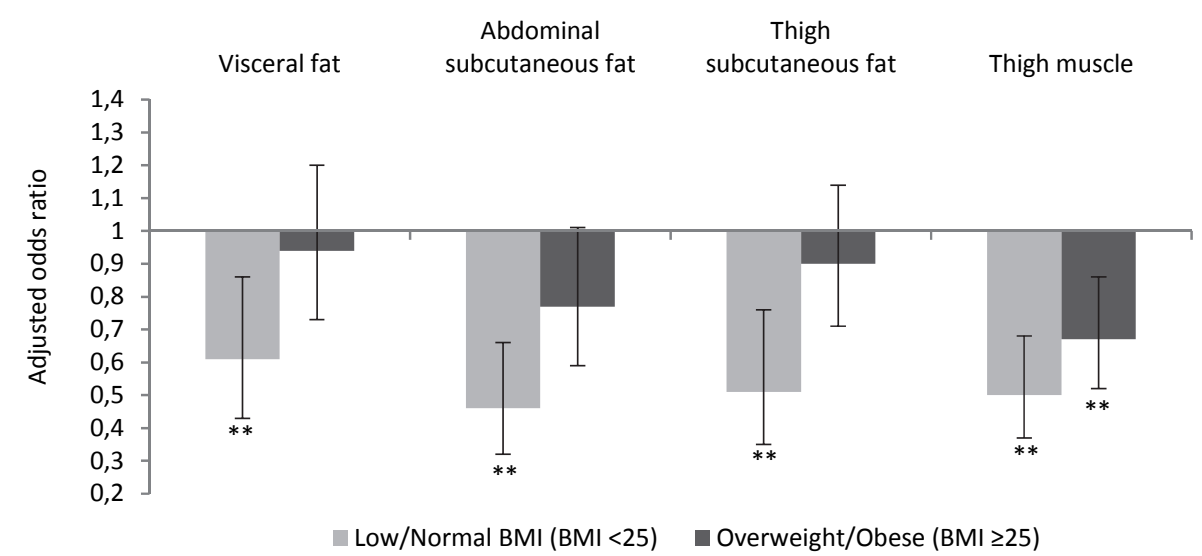

Figure 5.3 Associations between body composition and cognitive status in women stratified by midlife BMI category. Bars are odds ratios for dementia per SD increase in visceral, abdominal and thigh subcutaneous fat, and muscle tissue, adjusted for age, educational level, smoking status, depressive symptoms, type 2 diabetes, hypertension, stroke, triglyceride/HDL cholesterol ratio, and high-sensitivity C-reactive protein (Model 2 ), and error bars are $95 \%$ confidence intervals. ${ }^{* *} P$-value $<0.01$.

\section{Discussion}

In this cross-sectional study of a large community-based cohort of older adults we found that higher amounts of abdominal subcutaneous fat, subcutaneous thigh fat and thigh muscle were associated with decreased odds of dementia in women; the association of visceral fat with dementia was not statistically significant. These associations were independent of demographics, vascular risk factors, hs-CRP, 
depression, stroke, and were not moderated by APOE \&4 status. Associations between fat and dementia were related to weight change from midlife (less weight loss/ more weight gain was association with a decreased likelihood of dementia in women), while thigh muscle was associated with dementia independent of the weight change from midlife. In men, only an increase in thigh muscle was associated with decreased odds of dementia, independent of weight change. Furthermore, the associations between BC, particularly thigh subcutaneous fat, and odds of dementia were stronger in women with a low to normal midlife $\mathrm{BMI}(\mathrm{BMI}<25)$ compared with those with a high midlife $\mathrm{BMI}$ ( $\mathrm{BMI} \geq 25$ ). This pattern was not evident in men.

Our results are in line with previous studies showing that a decline in body weight and a low late-life BMI are associated with dementia. ${ }^{5,6,22}$ In the current study the association between fat tissue and decreased odds of dementia was only found in women. Our measures of cognitive status, thigh muscle, visceral and subcutaneous fat, as well as our accounting for midlife weight history are novel approaches to understand relationships between $\mathrm{BC}$ and cognitive status and are different from other studies. This makes comparisons of findings with prior studies difficult. One study with similar BC measures found that higher levels of abdominal subcutaneous fat were associated with worsening cognitive function in older men. ${ }^{23}$ However, unlike our analysis, this study used change in score on the Modified Mental State Examination (a brief general cognitive test) as an outcome measure and did not examine associations with $\mathrm{MCl}$ or dementia; our findings suggest that the association between adiposity and cognition may be different in individuals who will develop dementia compared with those who will not. Other studies have shown that higher fat mass and muscle mass are associated with a lower risk of cognitive impairment in elderly women, which is more in line with the present study, although these studies did not separate visceral from abdominal subcutaneous fat and did not use a diagnosis of dementia as an outcome measure. ${ }^{24,25}$

The inverse associations between fat and dementia in women may reflect processes related to the regulation of female hormones. Adipose tissue is the major source of endogenous estrogens in postmenopausal women. ${ }^{26,27}$ Estrogens may affect cognition by binding to estrogen receptors that are located throughout the brain, especially in regions of the hippocampus and amygdala, which are involved in learning and memory. ${ }^{28}$ Indeed, studies have shown that endogenous estradiol is associated with a decreased risk of cognitive impairment. ${ }^{24,29}$ Another potential mechanistic pathway may involve the hormone leptin. Leptin is produced by adipose tissue, particularly by subcutaneous fat ${ }^{30}$ and has been associated with learning and memory processes and with lower dementia risk. ${ }^{4}$ Furthermore, some studies have suggested a possible beneficial role for thigh subcutaneous fat as more gluteofemoral fat tissue (fat in thigh and buttocks) has been associated with lower glucose and lipid levels and as loss of subcutaneous fat, observed in patients with lipodystrophic syndromes, has been associated with an increased risk for insulin resistance, diabetes, and dyslipidemia. ${ }^{31}$ However, in our study adjustment for TG/HDL cholesterol ratio, an 
indicator of insulin resistance ${ }^{18}$ and predictor of coronary heart disease, ${ }^{19}$ and diabetes did not change the association between thigh subcutaneous fat and dementia when entered separately in the model. This makes this explanation less likely.

Although hormonal and metabolic processes may be involved, our findings indicate that associations between adiposity and dementia in women are not independent of weight loss, which can precede diagnosis of dementia by several years ${ }^{6}$ and may be caused by neurodegenerative changes that disrupt the regulation of energy metabolism and food intake by the central nervous system. ${ }^{32}$ In addition, potential mediators including vascular risk factors, stroke, and hs-CRP did not explain associations of $\mathrm{BC}$ with dementia, which again may indicate that neurodegenerative changes are involved. Furthermore, in our sample, women with dementia lost more weight from midlife than men with dementia (-3.15\% vs. $-0.97 \%$, respectively), which may explain why we only found associations between adiposity and dementia in women.

Reduced thigh muscle tissue was independently associated with the likelihood of dementia in both men and women. This is in line with previous research showing that lean mass was reduced in individuals with early $A D$ and was associated with poorer cognition and brain atrophy. ${ }^{10}$ In contrast to fat, associations between low thigh muscle and dementia persisted even after adjustment for weight change. This suggests that the composition of weight loss from midlife was predominately fat, which is consistent with research showing that a decline in muscle mass is not always reflected in a decrease in body weight. ${ }^{33}$ The association between reduced muscle tissue and dementia may be explained by a decreased production of sex hormones and/or an increased production of inflammatory factors, which have both been associated with loss of muscle mass, ${ }^{34}$ and dementia. ${ }^{32,35}$ This may suggest that muscle loss and dementia share common mechanisms.

In this study, BC measures were not significantly associated with $\mathrm{MCl}$. Previous studies have shown inverse associations of fat and muscle mass with cognitive impairment ${ }^{24,25}$ but these studies did not use clinical outcomes and did not consider dementia patients as a separate group. Loss of fat and muscle tissue may be the result of neurodegenerative changes associated with dementia and it is possible that we did not find associations between $\mathrm{BC}$ and $\mathrm{MCl}$, because not all $\mathrm{MCl}$ patients develop dementia.

Data on midlife BMI in this study uniquely allowed us to stratify associations between $\mathrm{BC}$ measures and dementia by midlife BMI. Our finding that the association between more fat, particularly subcutaneous fat, and decreased odds of dementia was stronger in women with a low to normal midlife BMI than in women with a high midlife BMI may indicate that women with a high midlife BMI follow a different trajectory towards old age than women with a low to normal midlife BMI. In addition, midlife BMI seems to influence cross-sectional associations between $B C$ and cognitive status at late life. However, our results should be regarded as hypotheses-generating 
research and more research is needed to examine the longitudinal relationship between changes in $\mathrm{BC}$ and cognitive status.

To the best of our knowledge, this is the first study to examine associations between multiple $\mathrm{BC}$ measures, measured with $\mathrm{CT}$, and $\mathrm{MCl}$ and dementia in a large cohort stratified by sex and by midlife BMI. Further strengths include our data on midlife weight and BMI. However, our study is not without limitations. Due to the cross-sectional design we were not able to examine temporal relationships. In addition, we did not adjust for physical activity, a potential important factor in associations between $\mathrm{BC}$ and dementia, since in the current study physical activity was measured with a retrospective questionnaire that was likely less reliable in people with cognitive impairment.

In conclusion, subcutaneous, and not visceral fat, was associated with lower odds of dementia in women, which was attenuated by the degree of change in weight from midlife. A higher amount of thigh muscle was associated with lower odds of dementia in both men and women, independent of weight change. Furthermore, associations between $\mathrm{BC}$ and dementia were particularly strong in women with a low to normal midlife BMI (BMI <25). These findings suggest that $B C$ changes associated with dementia may be mainly attributed to subcutaneous fat and muscle tissue. In addition, this study indicates that it is important to monitor changes in weight when individuals, particularly women, enter old age and suggests that it is important to develop interventions and programs aimed at maintaining weight and BC as individuals progress towards old age. Such interventions may include dietary interventions and interventions to increase physical activity, like muscle strength training. 


\section{References}

1. Luchsinger JA, Gustafson DR. Adiposity and Alzheimer's disease. Curr Opin Clin Nutr Metab Care 2009;12:15-21

2. Cournot M, Marquie JC, Ansiau D, et al. Relation between body mass index and cognitive function in healthy middle-aged men and women. Neurology 2006;67:1208-14

3. Power BD, Alfonso H, Flicker L, Hankey GJ, Yeap BB, Almeida OP. Body adiposity in later life and the incidence of dementia: the health in men study. PLoS One 2011;6:e17902

4. Gustafson DR. Adiposity and cognitive decline: underlying mechanisms. J Alzheimers Dis 2012;30 Suppl 2:S97-112

5. Fitzpatrick AL, Kuller $\mathrm{LH}$, Lopez $\mathrm{OL}$, et al. Midlife and late-life obesity and the risk of dementia: cardiovascular health study. Arch Neurol 2009;66:336-42

6. Stewart R, Masaki K, Xue QL, et al. A 32-year prospective study of change in body weight and incident dementia: the Honolulu-Asia Aging Study. Arch Neurol 2005;62:55-60

7. Gallagher D, Visser M, Sepulveda D, Pierson RN, Harris T, Heymsfield SB. How useful is body mass index for comparison of body fatness across age, sex, and ethnic groups? Am J Epidemiol 1996;143:228-39

8. Carr DB, Utzschneider KM, Hull RL, et al. Intra-abdominal fat is a major determinant of the National Cholesterol Education Program Adult Treatment Panel III criteria for the metabolic syndrome. Diabetes 2004;53:2087-94

9. Goodpaster BH, Thaete FL, Simoneau JA, Kelley DE. Subcutaneous abdominal fat and thigh muscle composition predict insulin sensitivity independently of visceral fat. Diabetes 1997;46:1579-85

10. Burns JM, Johnson DK, Watts A, Swerdlow RH, Brooks WM. Reduced lean mass in early Alzheimer disease and its association with brain atrophy. Arch Neurol 2010;67:428-33

11. Harris TB, Launer L, Eiriksdottir G, et al. Age, Gene/Environment Susceptibility-Reykjavik Study: multidisciplinary applied phenomics. Am J Epidemiol 2007;165:1076-87

12. Chang $M$, Jonsson PV, Snaedal J, et al. The effect of midlife physical activity on cognitive function among older adults: AGES--Reykjavik Study. J Gerontol A Biol Sci Med Sci 2010;65:1369-74

13. American Psychiatric Association. Diagnostic and Statistical Manual of Mental Disorders, 4th Edition (DSM-IV). Washington DC, American Psychiatric Association, 1994

14. Lopez OL, Becker JT, Jagust WJ, et al. Neuropsychological characteristics of mild cognitive impairment subgroups. J Neurol Neurosurg Psychiatry 2006;77:159-65

15. Murphy RA, Nalls MA, Keller M, et al. Candidate gene association study of BMI-related loci, weight, and adiposity in old age. J Gerontol A Biol Sci Med Sci 2013;68:661-6

16. Johannesdottir F, Aspelund T, Siggeirsdottir K, et al. Mid-thigh cortical bone structural parameters, muscle mass and strength, and association with lower limb fractures in older men and women (AGESReykjavik Study). Calcif Tissue Int 2012;90:354-64

17. Yesavage JA, Brink TL, Rose TL, et al. Development and validation of a geriatric depression screening scale: a preliminary report. J Psychiatr Res 1982;17:37-49

18. McLaughlin T, Abbasi F, Cheal K, Chu J, Lamendola C, Reaven G. Use of metabolic markers to identify overweight individuals who are insulin resistant. Ann Intern Med 2003;139:802-9

19. Gaziano JM, Hennekens CH, O'Donnell CJ, Breslow JL, Buring JE. Fasting triglycerides, high-density lipoprotein, and risk of myocardial infarction. Circulation 1997;96:2520-5

20. World Health Organization. Definition, diagnosis and classification of diabetes mellitus and its complications: report of a WHO consultation. Part 1, Diagnosis and classification of diabetes mellitus. Geneva, World Health Organization, 1999

21. Gudnason V, Sigurdsson J.G., Steingrimsdottir L., Humphries S., Sigurdsson G. Association of apolipoprotein E polymorphism with plasma levels of high density lipoprotein and lipoprotein(a), and effect of diet in healthy men and women. Nutr Metab Cardiovasc Dis 1993;3:136-41

22. Knopman DS, Edland SD, Cha RH, Petersen RC, Rocca WA. Incident dementia in women is preceded by weight loss by at least a decade. Neurology 2007;69:739-46

23. Kanaya AM, Lindquist $\mathrm{K}$, Harris $\mathrm{TB}$, et al. Total and regional adiposity and cognitive change in older adults: The Health, Aging and Body Composition (ABC) study. Arch Neurol 2009;66:329-35 
24. Bagger YZ, Tanko LB, Alexandersen P, Qin G, Christiansen C. The implications of body fat mass and fat distribution for cognitive function in elderly women. Obes Res 2004;12:1519-26

25. Nourhashemi F, Andrieu S, Gillette-Guyonnet S, et al. Is there a relationship between fat-free soft tissue mass and low cognitive function? Results from a study of 7,105 women. J Am Geriatr Soc 2002;50:1796-801

26. Szymczak J, Milewicz A, Thijssen JH, Blankenstein MA, Daroszewski J. Concentration of sex steroids in adipose tissue after menopause. Steroids 1998;63:319-21

27. Newton CJ, Samuel DL, James VH. Aromatase activity and concentrations of cortisol, progesterone and testosterone in breast and abdominal adipose tissue. J Steroid Biochem 1986;24:1033-9

28. McEwen BS, Akama KT, Spencer-Segal JL, Milner TA, Waters EM. Estrogen effects on the brain: actions beyond the hypothalamus via novel mechanisms. Behav Neurosci 2012;126:4-16

29. Yaffe K, Lui LY, Grady D, Cauley J, Kramer J, Cummings SR. Cognitive decline in women in relation to non-protein-bound oestradiol concentrations. Lancet 2000;356:708-12

30. Van Harmelen V, Reynisdottir S, Eriksson P, et al. Leptin secretion from subcutaneous and visceral adipose tissue in women. Diabetes 1998;47:913-7

31. Manolopoulos KN, Karpe F, Frayn KN. Gluteofemoral body fat as a determinant of metabolic health. Int J Obes (Lond) 2010;34:949-59

32. Mrak RE, Griffin WS. Interleukin-1, neuroinflammation, and Alzheimer's disease. Neurobiol Aging 2001;22:903-8

33. Thomas DR. Loss of skeletal muscle mass in aging: examining the relationship of starvation, sarcopenia and cachexia. Clin Nutr 2007;26:389-99

34. Doherty TJ. Invited review: Aging and sarcopenia. J Appl Physiol (1985) 2003;95:1717-27

35. Barron AM, Pike CJ. Sex hormones, aging, and Alzheimer's disease. Front Biosci (Elite Ed) 2012;4: 976-97 


\section{Supplemental data}

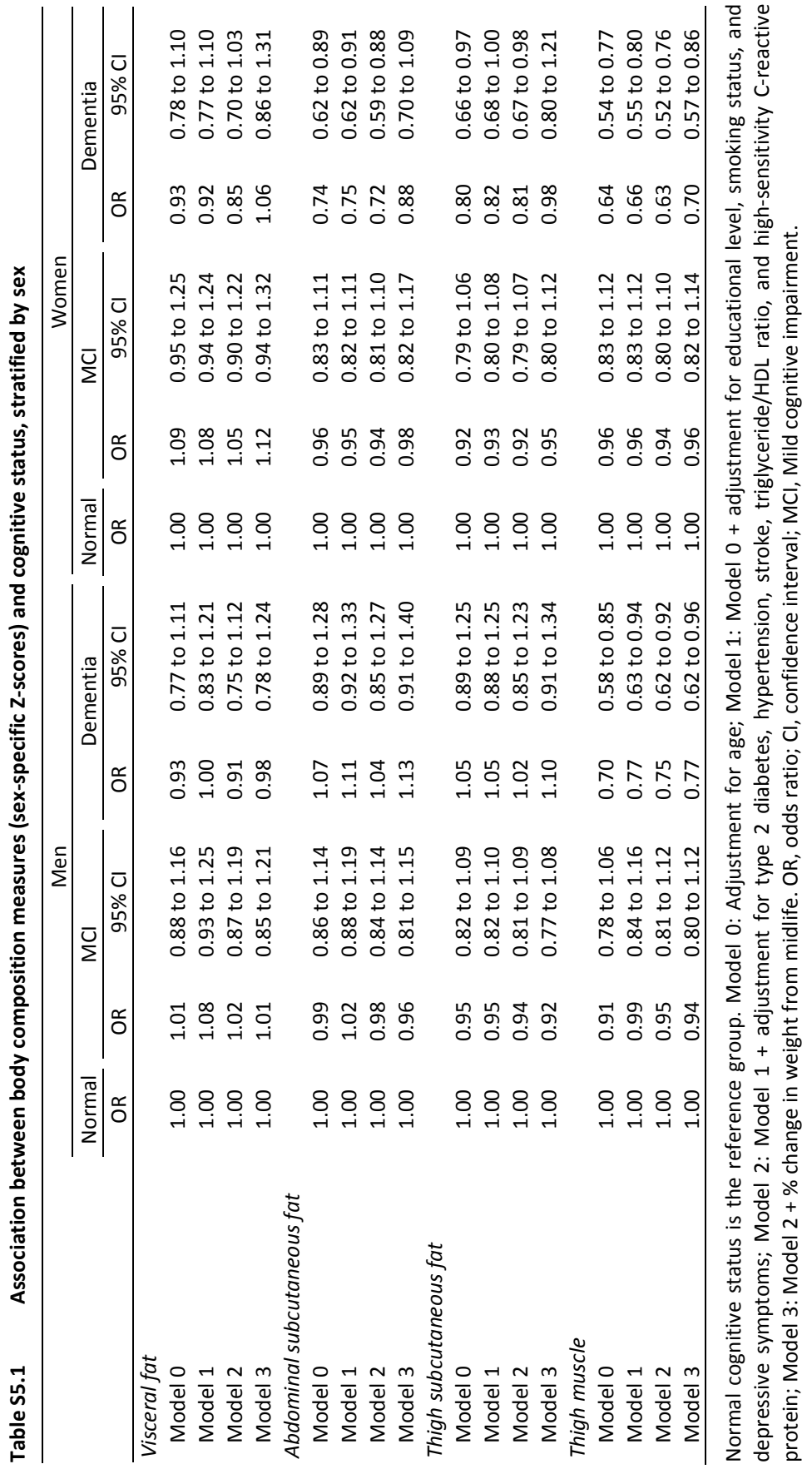




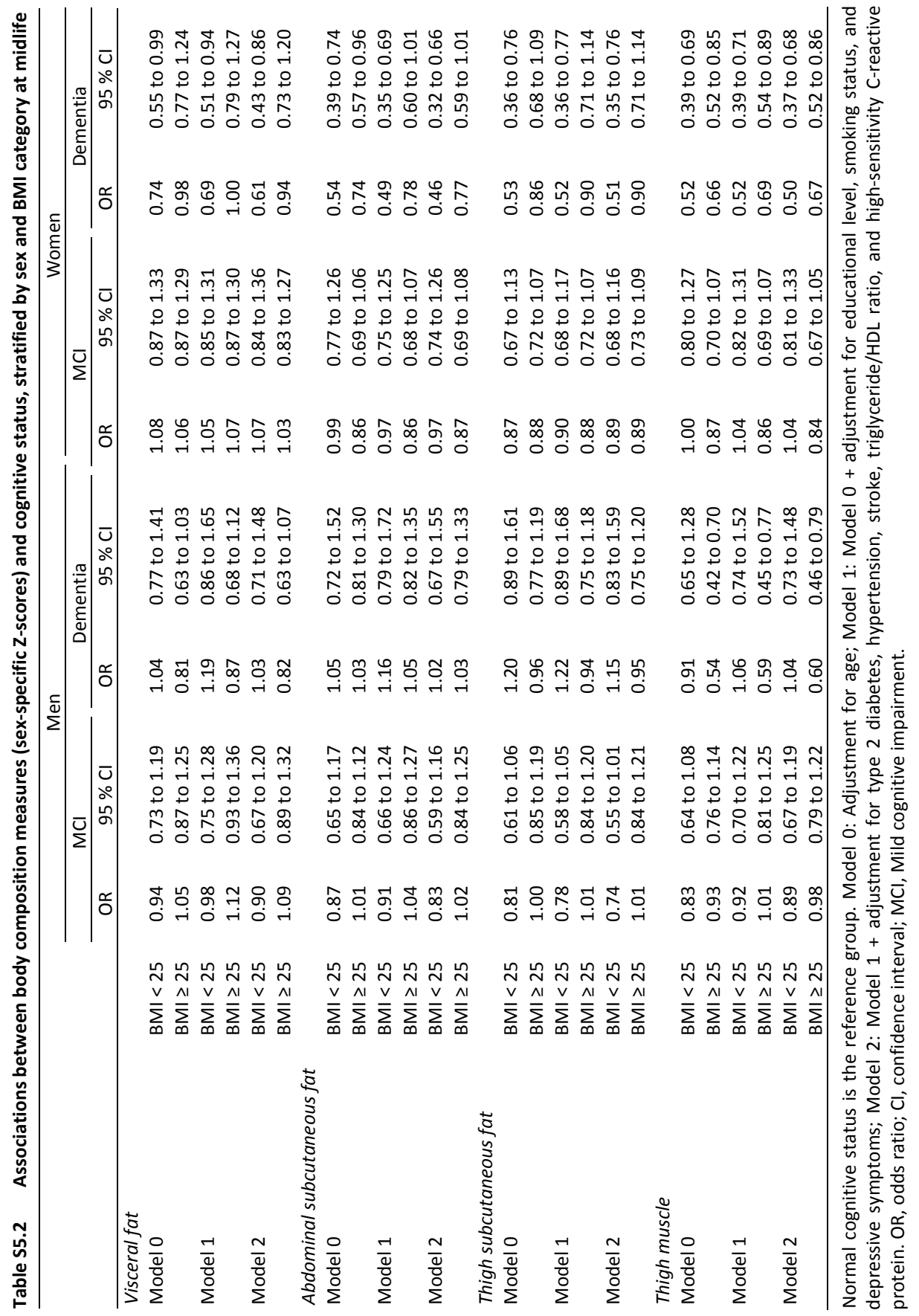




\section{Chapter 6}

LOWER VERBAL INTELLIGENCE IS ASSOCIATED

WITH DIABETIC COMPLICATIONS AND SLOWER

WALKING SPEED IN INDIVIDUALS WITH

TYPE 2 DIABETES: THE MAASTRICHT STUDY

PJJ Spauwen, RJH Martens, CDA Stehouwer, FRJ Verhey, MT Schram, SIS Sep,

CJH van der Kallen, PC Dagnelie, RMA Henry, NC Schaper, MPJ van Boxtel 


\section{Abstract}

\section{Objective}

To determine the association of verbal intelligence, as a core constituent of health literacy, with diabetic complications and walking speed in individuals with type 2 diabetes

\section{Methods}

This study was performed in 228 individuals with type 2 diabetes who participated in The Maastricht Study, an ongoing population-based cohort study. We examined cross-sectional associations of the score on the Word list test of the Groningen Intelligence Test with 1) determinants of diabetic complications (glycated hemoglobin A1c, blood pressure, and lipid level), 2) diabetic complications: chronic kidney disease (CKD) (estimated glomerular filtration rate $<60 \mathrm{ml} / \mathrm{min} / 1.73 \mathrm{~m}^{2}$ and/or a urinary albumin excretion of $\geq 30 \mathrm{mg}$ per 24 hours), neuropathic pain, self-reported history of cardiovascular disease (CVD), and carotid intimamedia thickness (c-IMT), and 3) walking speed. Analyses were adjusted in separate models for potential confounders (age and sex) and for potential mediators (vascular risk factors, lifestyle factors and diabetes-related variables). Significant age and sex-adjusted associations were additionally adjusted for educational level in a separate model.

\section{Results}

After adjustment for potential confounders and mediators a lower verbal intelligence was associated with the presence of neuropathic pain (OR 1.18, 95\% Cl 1.03 to 1.31), CVD (OR 1.14, $95 \% \mathrm{Cl} 1.01$ to 1.30 ), and with slower walking speed (regression coefficient $-0.011 \mathrm{~m} / \mathrm{s}$, $95 \% \mathrm{Cl}-0.021$ to $-0.002 \mathrm{~m} / \mathrm{s}$ ). These associations were largely explained by educational level. Verbal intelligence was not associated with blood pressure, glycemic control, lipid control, CKD or C-IMT.

\section{Conclusions}

A lower verbal intelligence was associated with the presence of some (but not all) diabetic complications and with a slower walking speed, a measure of physical functioning and important predictor of overall health status. Educational level largely explained these associations. This implies that awareness of clinicians for the educational level of their diabetic patients is important and information should be provided at a level of complexity tailored to the individual patient. 


\section{Introduction}

Patients with type 2 diabetes have to engage in self-care behaviors and make decisions that will determine successful treatment and development of long-term complications. These self-care behaviors and decisions involve lifestyle changes, the incorporation of medication-use into daily routine, and disease monitoring. Furthermore, patients need to interact with health care providers and diabetes educators. Health literacy, i.e. "the degree to which individuals have the capacity to obtain, process, and understand basic health information and services needed to make appropriate health decisions", ${ }^{1,2}$ may determine how well patients perform these activities and may therefore play a role in the development of long-term diabetic complications.

Low health literacy is common in diabetes ${ }^{3,4}$ and has been associated with problems reading prescriptions, ${ }^{5}$ fewer self-care behaviors, ${ }^{3,6}$ and poorer disease knowledge. ${ }^{3}$ Therefore, low health literacy may have a negative effect on glycemic control and diabetic complications, as is illustrated by some ${ }^{4,7,8}$ but not all ${ }^{3,9,10}$ studies. These discrepancies may be due to differences in glycemic control between studies and the use of different measures of health literacy.

Health literacy is often assessed by measures of verbal ability, ${ }^{11}$ which are related to verbal intelligence and have been associated with educational level. ${ }^{12,13}$ Verbal intelligence, as assessed by vocabulary tests, has been suggested to be a core constituent and a strong determinant of health literacy. ${ }^{12}$ Furthermore, verbal intelligence has been associated with health care compliance in individuals at high risk of cardiovascular disease. ${ }^{12}$ Therefore verbal intelligence may be associated with diabetic complications.

Intelligence is a strong predictor of educational level ${ }^{14,15}$ and educational attainment has been suggested to represent a proxy for intelligence. ${ }^{15}$ Furthermore, lower educational level has been associated with diabetic complications, ${ }^{16}$ and has been shown to strongly attenuate the association between childhood IQ and risk factors for premature death in middle-aged individuals. ${ }^{15}$ It is therefore important to examine whether the association between verbal intelligence and diabetic complications is independent of educational level, a variable that is less timeconsuming and easier to assess in daily practice.

In the current study we tested the hypothesis that lower verbal intelligence is associated with the presence of complications in type 2 diabetes. We first examined associations of verbal intelligence, measured with a standard vocabulary test, with glycemic control, systolic blood pressure, and cholesterol levels, which are important targets in the management of diabetes. ${ }^{17,18}$ Second, we tested associations of verbal intelligence with microvascular complications (chronic kidney disease (CKD) and neuropathic pain), macrovascular complications (carotid intima-media thickness (c-IMT), and history of cardiovascular disease), and walking speed during a 6 minutes walking test, a measure of physical functioning and predictor of overall health 
status. ${ }^{19,20}$ Third, we examined whether these associations were independent of lifestyle, vascular risk factors, depression, and diabetes-related factors (i.e. glycemic control, diabetes duration, and glucose-lowering medication use). Finally, we examined the extent to which these associations were explained by educational level.

\section{Research design and methods}

\section{Study population and design}

In this study, we used data from The Maastricht Study, an observational prospective population-based cohort study. The rationale and methodology have been described previously (21). In brief, the study focuses on the etiology, pathophysiology, complications and comorbidities of type 2 diabetes mellitus and is characterized by an extensive phenotyping approach. Eligible for participation were all individuals aged between 40 and 75 years and living in the southern part of the Netherlands. Participants were recruited through mass media campaigns and from the municipal registries and the regional Diabetes Patient Registry via mailings. Recruitment was stratified according to known diabetes status for reasons of efficiency. The present report includes cross-sectional data from the first 253 participants with type 2 diabetes, who completed the baseline survey between November 2010 and March 2012. The examinations of each participant were performed within a time window of three months. The study has been approved by the institutional medical ethical committee (NL31329.068.10) and the Minister of Health, Welfare and Sports of the Netherlands, on the basis of the Health Council's opinion (Permit 131088-105234-PG). All participants gave written informed consent.

\section{Glucose metabolism status}

To determine glucose metabolism, all participants (except those who used insulin) underwent a standardized 7-point oral glucose tolerance test after an overnight fast as previously described. ${ }^{21}$ Type 2 diabetes was defined according to the World Health Organization 2006 criteria. $^{22}$ Individuals without type 1 diabetes and on diabetes medication were considered as having type 2 diabetes. ${ }^{21}$

\section{Independent variable}

Verbal intelligence was measured with the Word list test of the Groningen Intelligence Test (GIT), ${ }^{23}$ which has been suggested to be a core constituent and strong determinant of health literacy. ${ }^{12}$ In this test, which consists of 20 items, participants are asked to indicate which of the five alternative words is exactly synonymous with a given word. Scores on this test range from 0 to 20. Performance on vocabulary tests, like the GIT Word list, peaks in midlife and deteriorates little with age, and is therefore 
an indication of a person's level of peak prior ability. ${ }^{24}$ In addition, vocabulary represents the level of skills that are relatively resistant to neurological injury. ${ }^{13,25}$

\section{Dependent variables}

\section{Glycemic, blood pressure, and LDL-cholesterol control}

Fasting blood samples of all participants were collected and glycated hemoglobin A1c $(\mathrm{HbA} 1 \mathrm{c})$, total low-density lipoprotein (LDL) and high-density lipoprotein (HDL) cholesterol were determined as described elsewhere. ${ }^{21}$ Office blood pressure was determined three times on the right arm after a 10-minute rest period, using a noninvasive blood pressure monitor (Omron 705IT, Omron, Japan). When the difference between measurement two and three was more than $10 \mathrm{mmHg}$, a fourth measurement was performed. All available measurements were used to calculate the average blood pressure. $^{21}$

\section{Diabetic complications}

Information was obtained on microvascular complications, i.e. CKD and neuropathic pain, and on macrovascular complications (history of cardiovascular disease and c-IMT) as described below.

\section{Chronic Kidney Disease}

Participants with an estimated glomerular filtration rate (eGFR) below $60 \mathrm{ml} / \mathrm{min} / 1.73 \mathrm{~m}^{2}$ and/or (micro)albuminuria were regarded as having CKD. ${ }^{26} \mathrm{GFR}$ was estimated using the CKD-EPI equation based on both serum creatinine and serum cystatin $\mathrm{C}^{27}$ Serum creatinine was measured as described elsewhere. ${ }^{21}$ Serum cystatin $C$ was measured with a particle enhanced immunoturbidimetric assay standardized against ERM-DA471/IFCC reference material (Roche Cobas 8000, F. Hoffman-La Roche Ltd, Basel, Switzerland). To assess urinary albumin excretion, participants were requested to collect two 24-hour urine collections. Urinary albumin concentration was measured with a standard immunoturbidimetric assay by an automatic analyzer (Beckman Synchron LX20, Beckman Coulter Inc., Brea, USA) and multiplied by collection volume to obtain the 24-hour urinary albumin excretion. A urinary albumin concentration below the detection limit of the assay $(2 \mathrm{mg} / \mathrm{l})$ was set at $1.5 \mathrm{mg} / \mathrm{l}$ before multiplying by collection volume. Only urine collections with a collection time between 20 and 28 hours were considered valid. If needed, urinary albumin excretion was extrapolated to a 24-hours' excretion. Microalbuminuria was defined as a urinary albumin excretion of 30-300 mg per 24 hours whereas macroalbuminuria was defined as a urinary albumin excretion of $>300 \mathrm{mg}$ per 24 hours. ${ }^{26}$ These definitions were based on one (11.1\%) or preferably the average of two (88.9\%) 24-hour urine collections. In a sensitivity analysis, only those participants with two 24-hour urine collections were included. 


\section{Neuropathic pain}

All participants received the question "Do you have pain or unpleasant feelings (e.g. tingling, burning or electric shocks) in your feet or lower legs?" If present they were asked to describe this complaint according to the Douleur Neuropathique 4 (DN4) interview. ${ }^{28}$ The total score of this questionnaire is based on the sum of the responses (minimum score is 0 and maximum score is 7 ). Scores $\geq 3$ are considered to be indicative of neuropathic pain. ${ }^{28-30}$

\section{History of cardiovascular disease:}

History of cardiovascular disease was defined as self-reported: myocardial infarction, and/or cerebrovascular infarction or hemorrhage, and/or percutaneous artery angioplasty of, or vascular surgery on, the coronary, abdominal, peripheral or carotid arteries.

\section{Carotid intima-media thickness}

All c-IMT measurements were done by trained vascular technicians unaware of the participants' clinical status, in a dark, quiet temperature controlled room $\left(21-23^{\circ} \mathrm{C}\right)$. The measurements were performed in supine position after 10 minutes of rest. Talking or sleeping was not allowed during the examination. Measurements were done at the left common carotid artery $10 \mathrm{~mm}$ proximal to the carotid bulb with the use of an ultrasound scanner equipped with a 7.5-MHz linear probe (MyLab 70, Esaote Europe, Maastricht, the Netherlands). This setup enables the measurement of $\mathrm{IMT}{ }^{31,32}$ Briefly, during the ultrasound measurement a B-mode image on the basis of $19 \mathrm{M}$-lines was depicted on screen and an online echo-tracking algorithm showed real-time anterior and posterior arterial wall displacements. The M-mode recordings were composed of 19 simultaneous recordings at a frame rate of $498 \mathrm{~Hz}$. The distance between the $\mathrm{M}$-line recording positions was $0.96 \mathrm{~mm}$, thus, a total segment of $18.24 \mathrm{~mm}$ of each artery was covered by the scan plane. For off-line processing, the radiofrequency signal was fed into a dedicated PC-based acquisition system (ART.LAB, Esaote Europe, Maastricht, the Netherlands) with a sampling frequency of $50 \mathrm{MHz}$. Data processing was performed in MatLab (version 7.5, Mathworks, Natick, MA, USA). Mean carotid IMT was calculated along the total scan plane, and was defined as the distance of the posterior wall from the leading edge interface between lumen and intima to the leading edge interface between media and adventitia. ${ }^{32}$ The median IMT of three measurements was used in the statistical analyses. Reproducibility was assessed in 12 individuals ( 6 men; $60.8 \pm 6.8$ years; 6 with type 2 diabetes) who were examined by two observers at two occasions spaced one week apart. The intra- and inter-observer intra-class correlation coefficients were 0.90 and 0.91 , respectively. 


\section{Walking speed}

Walking speed was assessed with a fast 6-minute walk test. Participants were instructed to walk from one end to the other of a 20-meter hallway as fast as possible, while attempting to cover as much ground as possible in the allotted 6 minutes. Exclusion criteria for this test included use of an assistive device for walking $(n=2)$, a heart rate $<40$ or $>110$ beats per minute, systolic blood pressure $\geq 180 \mathrm{mmHg}$, or diastolic blood pressure $\geq 110 \mathrm{mmHg}(\mathrm{n}=10)$; reported heart attack, angioplasty of the coronary arteries, or heart surgery within the last 3 months or reported chest pain, shortness of breath, or fainting in the past 3 months $(n=11)$. Furthermore, 3 participants started the test but did not finish it and 23 participants were excluded for another reason or the reason for exclusion was unknown. Walking speed $(\mathrm{m} / \mathrm{s})$ was calculated by dividing the total distance covered by six minutes.

\section{Covariates}

Smoking status (never, former, current), alcohol consumption, diabetes duration, and physical activity (hours of moderate to vigorous physical activity per week) were assessed by questionnaire, and lipid-modifying, antihypertensive, and glucoselowering medication use during an interview. ${ }^{21}$ Diabetes duration (years) was calculated as the difference between the year of study inclusion minus self-reported year of diabetes onset. For newly diagnosed diabetic patients the diabetes duration was therefore 0 years. Waist circumference was assessed as described elsewhere. ${ }^{21}$ Alcohol consumption was classified into three categories: non-consumption, lowconsumption ( $\leq 7$ and $\leq 14$ glasses per week for females and males) and highconsumption ( $>7$ glasses and $>14$ glasses per week for females and males). Level of education was assessed by interview and was classified into 8 categories commonly used in the Netherlands $\left.{ }^{33}: 1\right)$ no education, 2) primary education, 3) lower vocational education, 4) intermediate general secondary education, 5) intermediate vocational education, 6) higher general secondary education, 7) higher vocational education, 8 ) university degree. For this study, three groups were made for educational level: low (level 1 to 3), intermediate (level 4 to 6), and high (level 7 to 8). Presence of depression was assessed by the Mini International Neuropsychiatric Interview. ${ }^{21,34}$

\section{Statistical analysis}

Analyses were conducted using the SPSS software, version 20 for Mac OSX. Scores on the GIT wordlist test were reversed such that a lower score on the wordlist could be interpreted as a risk factor in the analyses. Differences in characteristics between tertiles of wordlist score were tested using analysis of variance for continuous variables and Chi-square tests for categorical variables. If assumptions were not met for the Chi-square test (expected count $<5$ ), Fisher's Exact test was used. Multiple linear regression analyses were performed to estimate the association of verbal 
intelligence with continuous outcomes, logistic regression analyses for associations with binary outcomes, and multinomial regression analyses for associations with categorical outcomes. To maximize the sample size per outcome, we conducted complete case analyses per outcome.

Associations were adjusted for different sets of potential confounders/mediators in separate models. In Model 1, no covariate adjustments were made and in Model 2, associations were adjusted for potential confounders (age and sex). If associations were significant in Model 2, associations were further adjusted for potential mediators in subsequent models. In Model 3, we additionally adjusted for smoking status, alcohol consumption, waist circumference, total cholesterol/HDL cholesterol ratio, triglyceride levels, systolic blood pressure, lipid-modifying and/or antihypertensive medication use, and depression. In Model 4, we additionally adjusted for diabetes-related variables (HbA1c level, diabetes duration, and glucoselowering medication use). Significant associations in Model 2 were adjusted for educational level in Model 5 (Model 2+educational level) to examine whether these associations could be explained by educational level. We calculated the percentage change in regression coefficient (from Model 2 to Model 5) to estimate which part of the association was explained by educational level using the following formula ${ }^{35,36}$ : ( $\left(\right.$ regression coefficient $\left.B_{\text {verbal intelligence Model } 2}-B_{\text {verbal intelligence Model } 5}\right) / B_{\text {verbal intelligence Model } 2 \text { ) }}$ $x 100 \%$

Information on physical activity was only available for 179 participants ( $21.5 \%$ of the 228 participants had missing data). Therefore we performed separate analyses including moderate to vigorous physical activity as an additional potential mediator (Model 3+physical activity). For all analyses a $P$-value of $<0.05$ was considered statistically significant in two-sided tests.

\section{Results}

\section{Sample}

Of the 253 participants with type 2 diabetes, 3 participants had missing data on GIT word list score and 22 on potential confounders/mediators, thus 25 participants were excluded from all analyses. This resulted in 228 participants for analyses with HbA1c and SBP and 224 for analyses with LDL-cholesterol as outcome. Additionally, individuals with missing data on diabetic complications and walking speed were excluded. In the remaining sample of 228 participants, the number of participants with missing data on diabetic complications ranged from 7 to 19, and 40 participants had missing data on walking speed. This resulted in 221, 209, 219, 218, and 188 participants for analyses with history of cardiovascular disease, neuropathic pain, CKD, c-IMT, and walking speed as outcomes, respectively. 
General characteristics of participants included in at least one of the analyses $(n=228)$ stratified by tertile of verbal intelligence (GIT wordlist score) are shown in Table 6.1.

Table 6.1 Characteristics of the study population stratified by tertiles of verbal intelligence $(n=228)$

\begin{tabular}{|c|c|c|c|c|}
\hline & Low $(n=73)$ & Medium (n=94) & High $(n=61)$ & $P$-value \\
\hline Word List score, & $10.6(1.8)$ & $14.1(0.8)$ & $17.0(0.9)$ & - \\
\hline Age, (years) & $63.7(6.7)$ & $63.7(7.6)$ & $63.3(6.6)$ & 0.93 \\
\hline Sex (men), n (\%) & $54(74.0)$ & $59(62.8)$ & $43.4(80.3)$ & 0.05 \\
\hline Educational level, n (\%) & $41 / 30 / 2$ & $20 / 46 / 28$ & $1 / 30 / 30$ & $<0.001$ \\
\hline low/middle/high & $(56.2 / 41.1 / 2.7)$ & $(21.3 / 48.9 / 29.8)$ & $(1.6 / 49.2 / 49.2)$ & \\
\hline Alcohol consumption, $\mathrm{n}(\%)$ & $23 / 42 / 8$ & $30 / 43 / 21$ & $10 / 34 / 17$ & 0.04 \\
\hline none/low/high & $(31.5 / 57.5 / 11.0)$ & $(31.9 / 45.7 / 22.3)$ & $(16.4 / 55.7 / 27.9)$ & \\
\hline Smoking status, n (\%) & $12 / 47 / 14$ & $26 / 60 / 8$ & $13 / 38 / 10$ & 0.20 \\
\hline never/former/current & $(16.4 / 64.4 / 19.2)$ & $(27.7 / 63.8 / 8.5)$ & $(21.3 / 62.3 / 16.4)$ & \\
\hline Waist circumference (cm) & 106.9 (11.9) & $106.4(14.3)$ & $103.7(12.0)$ & 0.32 \\
\hline Systolic blood pressure (mmHg) & $145.8(18.9)$ & $145.4(18.7)$ & $148.5(19.4)$ & 0.58 \\
\hline Antihypertensive medication use, $\mathrm{n}(\%)$ & $56(76.7)$ & $63(67.0)$ & $44(72.1)$ & 0.39 \\
\hline Lipid-modifying medication use, $\mathrm{n}(\%)$ & $59(80.8)$ & $67(71.3)$ & $46(75.4)$ & 0.36 \\
\hline Depression, n (\%) & $7(9.6)$ & $3(3.2)$ & $4(6.6)$ & 0.23 \\
\hline HbA1c level (\%), median (IQR) & $6.7(6.4-7.4)$ & $6.7(6.3-7.2)$ & $6.9(6.5-7.2)$ & $0.25^{\mathrm{c}}$ \\
\hline HbA1c level (mmol/mol), median (IQR) & $50.0(45.5-57.0)$ & $50.0(46.0-55.0)$ & $52.0(48.0-56.0)$ & \\
\hline Glucose-lowering medication, $\mathrm{n}(\%)$ & $9 / 44 / 20$ & $23 / 57 / 14$ & $16 / 35 / 10$ & 0.10 \\
\hline none/oral ${ }^{\mathrm{a}} /$ insulin ( \pm oral) & $(12.3 / 60.3 / 27.4)$ & $(24.5 / 60.6 / 14.9)$ & $(26.2 / 57.4 / 16.4)$ & \\
\hline Diabetes diagnosed at study entry, $\mathrm{n}(\%)$ & $8(11.0)$ & $17(18.1)$ & $11(18.0)$ & 0.39 \\
\hline Diabetes duration (years), median (IQR) & $7.0(2.5-11.5)$ & $4.5(1.0-10.0)$ & $5.0(2.0-9.5)$ & $0.22^{\mathrm{C}}$ \\
\hline Total cholesterol (mmol/l) & $4.29(0.86)$ & $4.49(1.02)$ & $4.55(1.06)$ & 0.12 \\
\hline HDL cholesterol (mmol/l) & $1.08(0.28)$ & $1.14(0.36)$ & $1.08(0.31)$ & 0.41 \\
\hline LDL-cholesterol (mmol/I) & $2.46(0.77)$ & $2.55(0.90)$ & $2.66(0.94)$ & 0.21 \\
\hline Total cholesterol/HDL cholesterol ratio & $4.1(1.0)$ & $4.2(1.2)$ & $4.4(1.0)$ & 0.35 \\
\hline Triglycerides (mmol/I), median (IQR) & $1.59(1.15-2.27)$ & $1.68(1.08-2.34)$ & $1.69(1.19-2.41)$ & $0.64^{c}$ \\
\hline $\begin{array}{l}\text { Moderate to vigorous physical activity } \\
\text { (hours per week), median (IQR) }\end{array}$ & $3.0(0.8-5.3)$ & $3.8(2.5-7.4)$ & $4.5(2.0-8.4)$ & $0.01^{\mathrm{c}}$ \\
\hline
\end{tabular}

\footnotetext{
${ }^{a}$ Two participants in this group used glucagon-like peptide-1 receptor agonists in addition to oral glucoselowering medication. ${ }^{\mathrm{b}}$ Available for 179 participants. ${ }^{\mathrm{c}} P$-values were derived from analysis with the nonparametric Kruskal-Wallis test. HbA1c, glycated hemoglobin A1c; HDL, high-density lipoprotein, LDL, lowdensity lipoprotein; IQR, interquartile range. Data are presented as mean (SD), unless otherwise stated.
}

Excluded participants $(n=25)$ did not differ statistically significantly in general characteristics from participants included, except for physical activity levels, which were significantly lower in excluded participants. Mean age of the study sample was 63.6 years $(S D=7.1)$ and the majority were men $(71.1 \%)$. Individuals had a median HbA1c level of $6.8 \%$, (IQR 6.4 to 7.2 ) (50.5 mmol/mol, IQR 47.0 to 55.0 ) mean systolic blood pressure of $146 \mathrm{mmHg}(\mathrm{SD}=19)$, and mean LDL-cholesterol level of $2.55 \mathrm{mmol} / \mathrm{l}$ $(S D=0.87)$. The majority of the sample was under good glycemic control $(62.3 \%$ of the sample had an $\mathrm{HbA} 1 \mathrm{c}<7.0 \%(<53 \mathrm{mmol} / \mathrm{mol}))$. The median diabetes duration was 5.0 years (IQR 2 to 10.75). Of the 228 participants $30.8 \%(n=68)$ reported a history of 
cardiovascular disease, $22.0 \%(n=46)$ neuropathic pain, $24.2 \%(n=53)$ had CKD, and mean c-IMT was $0.89 \mathrm{~mm}(\mathrm{SD}=0.15)$. Mean walking speed was $1.5 \mathrm{~m} / \mathrm{s}(\mathrm{SD}=0.2)$.

\section{Verbal intelligence and glycemic, blood pressure, and LDL control}

In both unadjusted and adjusted regression analyses (for age and sex), verbal intelligence score was not significantly associated with $\mathrm{HbA1c}$ level, systolic blood pressure, or LDL-cholesterol level. The regression coefficients (expressed per one word decrease on the Word list test) after adjustment for age and sex were -0.01 (95\% confidence interval $(\mathrm{Cl})-0.05$ to $0.03, P=0.76)$ for glycemic control, $-0.50(95 \% \mathrm{Cl}$ -1.39 to $0.39, P=0.28)$ for systolic blood pressure, and $-0.01(95 \% \mathrm{Cl}-0.05$ to 0.03 , $P=0.72$ ) for LDL-cholesterol.

\section{Verbal intelligence, diabetic complications, and walking speed}

In crude analyses, a lower verbal intelligence score was significantly associated with the presence of neuropathic pain (odds ratio (OR) 1.16, 95\% confidence interval (95\% CI) 1.03 to 1.31) and cardiovascular disease (OR 1.12, 95\% Cl 1.00 to 1.24) (Table 6.2, Model 1). These associations remained largely unchanged after adjustment for sex and age (Model 2, Figure 6.1). In contrast, verbal intelligence was not associated with CKD and C-IMT in both unadjusted and adjusted analyses. For analyses with CKD, excluding participants with only one 24-hour urine collection $(n=22)$ did not yield different results (data not shown). Associations of verbal intelligence with neuropathic pain and cardiovascular disease remained unchanged after additional adjustment for lifestyle factors, vascular risk factors, lipid-modifying and antihypertensive medication use, and depression (Table 6.2, Model 3). Furthermore, additional adjustment for diabetes-related factors did not materially change these associations (Table 6.2, Model 4). 


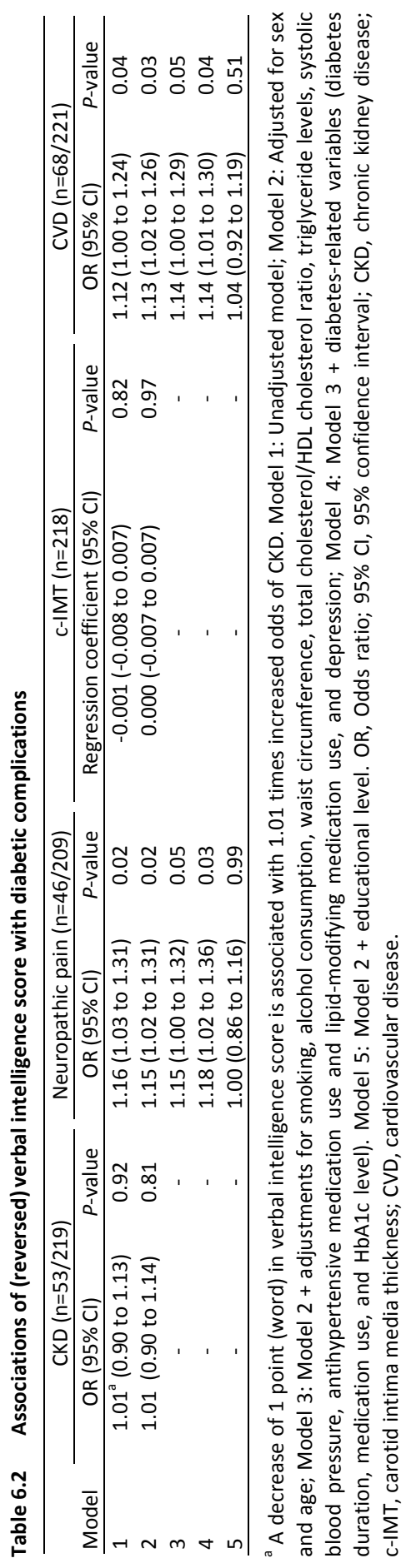


A lower verbal intelligence score was significantly associated with having a lower educational level $(\mathrm{OR} 1.46,95 \% \mathrm{Cl} 1.23$ to 1.73 for medium compared with high educational level; $\mathrm{OR} 2.34,95 \% \mathrm{Cl} 1.86$ to 2.94 for low compared with high educational level) after adjustment for age and sex. After adding educational level to Model 2, associations of verbal intelligence with neuropathic pain and cardiovascular disease were attenuated and were no longer significant (Model 5, for neuropathic pain: OR 1.00, 95\% Cl 0.86 to 1.16; for cardiovascular disease: OR 1.04, 95\% Cl 0.92 to 1.19). In this model, a lower educational level was significantly associated with the

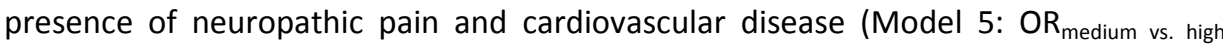
educational level $4.70,95 \% \mathrm{Cl} 1.30$ to $16.95 ; \mathrm{OR}_{\text {low vs. high educational level }} 11.27,95 \% \mathrm{Cl} 2.71$ to 46.77 for neuropathic pain; $\mathrm{OR}_{\text {medium vs. high educational level }} 1.4895 \% \mathrm{Cl} 0.65$ to $3.37 ; \mathrm{OR}_{\text {low vs. }}$ high educational level $2.95,95 \% \mathrm{Cl} 1.07$ to 8.11 for cardiovascular disease). Educational level explained $100 \%$ and $65 \%$ of the associations between verbal intelligence and neuropathic pain and cardiovascular disease, respectively.

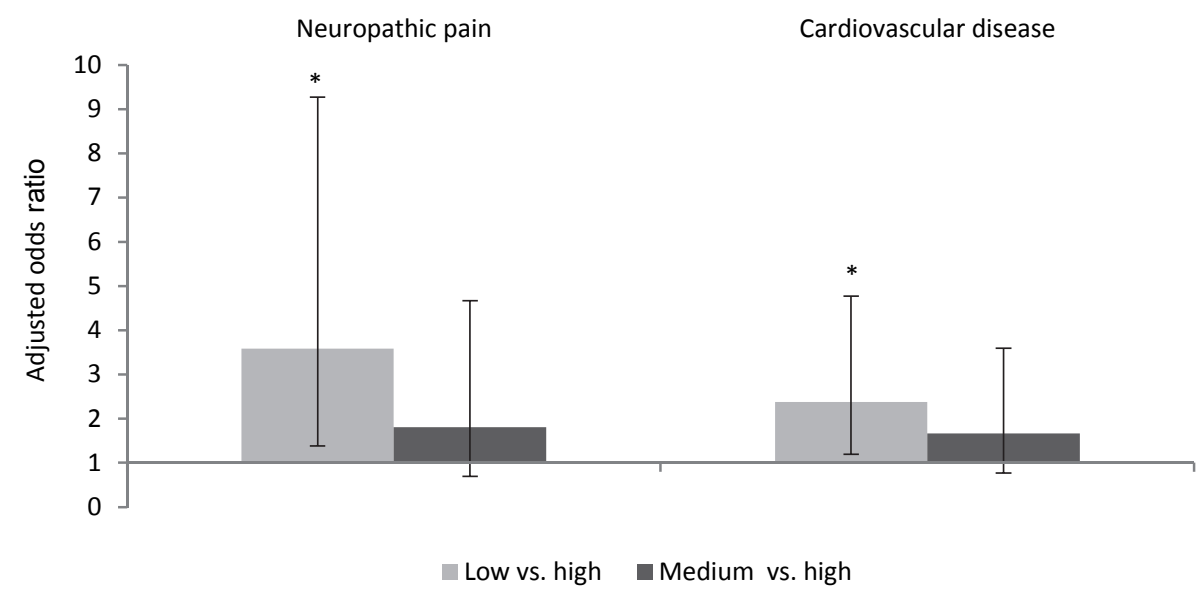

Figure 6.1 Associations of tertiles of verbal intelligence (medium and low compared with high) with neuropathic pain and cardiovascular disease, adjusted for age and sex. Bars are odds ratios and error bars are $95 \%$ confidence intervals. $* P<0.05$.

Lower verbal intelligence was associated with slower walking speed (regression coefficient b $-0.016,95 \% \mathrm{Cl}-0.027$ to -0.005 ) (Table 6.3, Model 1). After adjustment for age and sex the association remained largely unchanged (Table 6.3, Model 2). Associations of tertiles of verbal intelligence with walking speed in Model 2 are depicted in Figure 6.2. Adjustment for potential mediators slightly attenuated the association (Table 6.3, Model 3 and 4). Age and sex adjusted associations were attenuated and became non-significant after adjustment for educational level (Model 5: regression coefficient $-0.001,95 \% \mathrm{Cl}-0.013$ to 0.011 ) while a lower educational level was significantly associated with a slower walking speed in this model ( $b_{\text {medium vs. }}$ 
high educational level $-0.09,95 \% \mathrm{Cl}-0.16$ to $-0.03 ; b_{\text {low vs. high educational level }}-0.16,95 \% \mathrm{Cl}-0.25$ to 0.07). Educational level explained $92 \%$ of the association between verbal intelligence and walking speed.

Table 6.3 Associations of (reversed) verbal intelligence score with walking speed

\begin{tabular}{ccc}
\hline Model & Regression coefficient $(95 \% \mathrm{Cl})$ & $P$-value \\
\hline 1 & $-0.016^{\text {a }}(-0.027$ to -0.005$)$ & 0.00 \\
2 & $-0.013(-0.023$ to -0.003$)$ & 0.01 \\
3 & $-0.011(-0.020$ to -0.002$)$ & 0.02 \\
4 & $-0.011(-0.021$ to -0.002$)$ & 0.02 \\
5 & $-0.001(-0.013$ to 0.011$)$ & 0.86 \\
\hline
\end{tabular}

${ }^{a}$ A decrease of 1 point (word) in verbal intelligence score is associated with a decrease of $0.016 \mathrm{~m} / \mathrm{s}$ in walking speed. Model 1: Unadjusted model; Model 2: Adjusted for sex and age; Model 3: Model $2+$ adjustments for smoking, alcohol consumption, waist circumference, total cholesterol/HDL cholesterol ratio, triglyceride levels, systolic blood pressure, antihypertensive medication use and lipid-modifying medication use, and depression; Model 4: Model 3 + diabetes-related variables (diabetes duration, medication use, and HbA1c level); Model 5: Model 2 + educational level. OR, Odds ratio; $95 \% \mathrm{Cl}, 95 \%$ confidence interval.

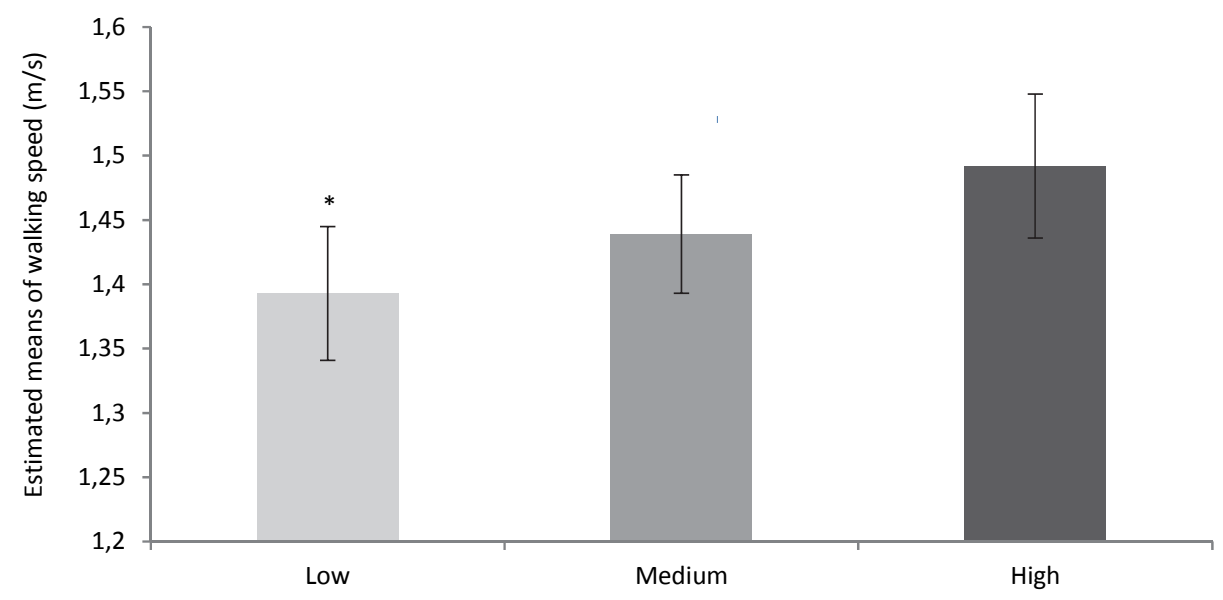

Figure 6.2 Estimated means of walking speed per tertile of verbal intelligence (low, medium, high), adjusted for age and sex. Bars indicate estimated means and error bars are $95 \%$ confidence intervals. * $P$ for comparison with high verbal intelligence $<0.05$.

\section{Additional analyses}

After adjustment for physical activity in subgroup analyses, associations between verbal intelligence on the one hand and neuropathic pain, cardiovascular disease, and walking speed on the other hand were largely unchanged (Model 3+adjustment for moderate to vigorous physical activity) (data not shown). 


\section{Discussion}

This cross-sectional study is the first to evaluate the associations of verbal intelligence with diabetic complications and walking speed in individuals with type 2 diabetes, and has three main findings. First, a lower verbal intelligence was associated with the presence of neuropathic pain and a history of cardiovascular disease, and with a slower walking speed, a measure of physical functioning and strong predictor of health status. ${ }^{19,20}$ Second, these associations were largely explained by educational level. Third, verbal intelligence was not associated with measures of glycemic, blood pressure, or LDL-cholesterol control as well as CKD and C-IMT.

To the best of our knowledge, no studies have examined associations of verbal intelligence with diabetic outcomes. Few studies have examined associations of health literacy with diabetic complications and have shown mixed results. ${ }^{4,9,37}$ These studies only included self-reported diabetic complications and most of these studies ${ }^{4,37}$ did not examine the role of cardiovascular risk factors and lifestyle factors in these associations.

Several explanations may be given for the lack of an association between verbal intelligence and the intermediate outcomes (glycemic, blood pressure and lipid control), as well as CKD and C-IMT in the current study. First, the intermediate outcomes were only measured at one moment, which may not accurately reflect longterm control. Previous studies for example have shown that historical glycemic control is a major determinant of diabetic complications, independent of current control. ${ }^{38}$

Second, patients with diabetes may change their lifestyles, affecting glycemic, blood pressure, and (or) lipid control, once they have developed severe complications, like cardiovascular disease and neuropathic pain, and are faced with the consequences of their disease. Furthermore, physicians may pay more attention to those patients who have developed complications and may intensify their medical treatment, as may be reflected by the more frequent use of antihypertensive and lipid-modifying medication in those with a history of cardiovascular disease in this population (data not shown). This may explain why we did not find associations with intermediate outcomes and why associations of verbal intelligence with diabetic complications and walking speed were independent of lifestyle factors and vascular risk factors. In general, diabetes care in The Netherlands has improved in the last couple of years, ${ }^{39,40}$ as may be reflected by the relatively good glycemic control in this study as compared with previous studies on health literacy. ${ }^{4,7}$ This also may have influenced associations of verbal intelligence with current glycemic, blood pressure, or lipid control.

Third, there is no single measure of health literacy that adequately addresses the full range of skills that define it. ${ }^{1}$ In the current study, an important determinant of health literacy was used, which may be less sensitive and specific than extensive health literacy measures like the S-TOFHLA ${ }^{41}$ and REALM $^{42}$ questionnaires which specifically assess comprehension or pronunciation of health-related terms. In 
addition, it has been suggested that numeracy, i.e. the ability to understand and use numbers, which is important in understanding medication dosing, test results, insulin requirements, and in interpreting food labels, ${ }^{1,43}$ is more strongly associated with glycemic control than literacy. ${ }^{6,43}$ As verbal intelligence may be less strongly related to numeracy than to literacy, associations of verbal intelligence with glycemic control as well as complications, which are, at least partly, related to glycemic control, may be less strong. Furthermore, it has been suggested that health literacy also encompasses social skills, ${ }^{44}$ which were not addressed in this study.

Interestingly, the associations of verbal intelligence with complications were largely explained by educational level indicating that educational level is an important mediator in these associations. Therefore it seems important to gather information about the educational level of patients with type 2 diabetes. Health literacy has been shown to mediate associations between educational level and glycemic control ${ }^{45}$ and has been suggested to mediate the relationship between educational level and heart failure in diabetic patients. ${ }^{37}$ We expand this finding by showing that educational level may be an important mediator in associations of verbal intelligence with neuropathic pain, cardiovascular disease, and walking speed. Verbal intelligence may determine health literacy and thereby the development of complications of diabetes at least partly through its influence on educational attainment. This implies that verbal intelligence causes differences in educational outcomes, which is plausible because verbal intelligence is highly heritable and large-scale longitudinal data have shown that verbal intelligence at age 11 strongly predicts educational outcomes at age $16 .^{14,46}$ However, it must be noted that education still may cause some intelligence differences. ${ }^{47}$ Furthermore, educational level may not only reflect an individual's capacity to understand health-related information, but is also an indicator of socioeconomic status, which may be linked to diabetic complications and physical functioning through social factors, including attitudes/beliefs and social support. ${ }^{48}$

\section{Strengths and limitations}

Our study has several strengths. We examined several health outcomes, including walking speed, several measures of diabetic complications, and their most important determinants, and we were able to adjust associations for multiple potential confounders/mediators. One limitation of this study is that we did not use a more extensive measure of health literacy. Instead, we tested an important determinant of health literacy that is easy to assess and relatively insensitive to the effects of brain damage. Furthermore, our results may have underestimated the true association of verbal intelligence with diabetic complications, as diabetic patients with severe complications and possibly a low verbal intelligence may not have participated in our study. Other limitations of this study include the relatively small sample size, particularly the small numbers of individuals with complications, and the crosssectional design. Unfortunately, due to later implementation of fundus photography 
in The Maastricht Study, retinal fundus images were only available for 74 patients and only 3 were diagnosed with retinopathy. Therefore we could not use retinopathy as an outcome measure.

\section{Conclusion and implications}

This study suggests that lower verbal intelligence is associated with the presence of diabetic complications and with a slower walking speed, a measure of physical functioning and strong predictor of health status, in individuals with type 2 diabetes. Educational level, which is less time-consuming and easier to assess than verbal intelligence, seems to be an important factor in these relationships. In order to reduce the risk of diabetic complications, it may therefore be important for clinicians to be well aware of the educational level of their patients in order to tailor the information to a level that can be understood.

Future studies should examine longitudinal associations of health literacy with glycemic, blood pressure, and lipid control over time and the development of diabetic complications. In addition, it is important to examine the role of educational level and verbal intelligence and their causal relationship with poor health literacy in order to develop strategies and tailored health education to increase healthy literacy, to reduce the risk of complications, and to increase health status in patients with type 2 diabetes. 


\section{References}

1. Bailey SC, Brega AG, Crutchfield TM, et al. Update on Health Literacy and Diabetes. Diabetes Educ 2014

2. Institute of Medicine. Health literacy: A Prescription to End Confusion. Washington DC, The National Academies, 2004

3. Williams MV, Baker DW, Parker RM, Nurss JR. Relationship of functional health literacy to patients' knowledge of their chronic disease. A study of patients with hypertension and diabetes. Arch Intern Med 1998;158:166-72

4. Schillinger D, Grumbach K, Piette J, et al. Association of health literacy with diabetes outcomes. JAMA 2002;288:475-82

5. Gazmararian JA, Baker DW, Williams MV, et al. Health literacy among Medicare enrollees in a managed care organization. JAMA 1999;281:545-51

6. Cavanaugh K, Huizinga MM, Wallston KA, et al. Association of numeracy and diabetes control. Ann Intern Med 2008;148:737-46

7. Powell CK, Hill EG, Clancy DE. The relationship between health literacy and diabetes knowledge and readiness to take health actions. Diabetes Educ 2007;33:144-51

8. Tang YH, Pang SM, Chan MF, Yeung GS, Yeung VT. Health literacy, complication awareness, and diabetic control in patients with type 2 diabetes mellitus. J Adv Nurs 2008;62:74-83

9. Morris NS, MacLean CD, Littenberg B. Literacy and health outcomes: a cross-sectional study in 1002 adults with diabetes. BMC Fam Pract 2006;7:49

10. DeWalt DA, Boone RS, Pignone MP. Literacy and its relationship with self-efficacy, trust, and participation in medical decision making. Am J Health Behav 2007;31 Suppl 1:S27-35

11. Boren SA. A review of health literacy and diabetes: opportunities for technology. J Diabetes Sci Technol 2009;3:202-9

12. Deary IJ, Gale CR, Stewart MC, et al. Intelligence and persisting with medication for two years: Analysis in a randomised controlled trial. Intelligence 2009;37:607-12

13. de Oliveira MO, Nitrini R, Yassuda MS, Brucki SM. Vocabulary is an appropriate measure of premorbid intelligence in a sample with heterogeneous educational level in Brazil. Behav Neurol 2014;2014:875960

14. Deary IJ, Strand S, Smith P, Fernandes C. Intelligence and educational achievement. Intelligence 2007;35:13-21

15. Batty GD, Deary IJ, Macintyre S. Childhood IQ in relation to risk factors for premature mortality in middle-aged persons: the Aberdeen Children of the 1950s study. J Epidemiol Community Health 2007;61:241-7

16. Chaturvedi N, Stephenson JM, Fuller JH. The relationship between socioeconomic status and diabetes control and complications in the EURODIAB IDDM Complications Study. Diabetes Care 1996;19: 423-30

17. Gaede P, Vedel P, Larsen N, Jensen GV, Parving HH, Pedersen O. Multifactorial intervention and cardiovascular disease in patients with type 2 diabetes. N Engl J Med 2003;348:383-93

18. American Diabetes A. Standards of medical care in diabetes--2014. Diabetes Care 2014;37 Suppl $1: \mathrm{S} 14-80$

19. Jylha M, Guralnik JM, Balfour J, Fried LP. Walking difficulty, walking speed, and age as predictors of self-rated health: the women's health and aging study. J Gerontol A Biol Sci Med Sci 2001;56:M609-17

20. Studenski S, Perera S, Wallace D, et al. Physical performance measures in the clinical setting. J Am Geriatr Soc 2003;51:314-22

21. Schram MT, Sep SJ, van der Kallen CJ, et al. The Maastricht Study: an extensive phenotyping study on determinants of type 2 diabetes, its complications and its comorbidities. Eur J Epidemiol 2014;29: 439-51

22. World Health Organization. Definition and Diagnosis of Diabetes Mellitus and Intermediate Hyperglycemia: Report of a WHO/IDF Consultation. Geneva, World Health Organization, 2006

23. Luiteijn F, Ploeg van der FAE. Handleiding Groninger Intelligentietest (GIT). Lisse, Swess and Zeitlinger, 1983 
24. Salthouse TA. Localizing age-related individual differences in a hierarchical structure. Intelligence 2004;32

25. Lezak MD, Howieson DB, Loring DW. The rationale of deficit measurement. In: Lezak MD, Howieson DB, Loring DW, (Eds.). Neuropsychological assessment. 4th ed. Oxford, Oxford University Press, 2004. p. 91-7

26. National Kidney F. K/DOQI clinical practice guidelines for chronic kidney disease: evaluation, classification, and stratification. Am J Kidney Dis 2002;39:S1-266

27. Inker LA, Schmid $\mathrm{CH}$, Tighiouart $\mathrm{H}$, et al. Estimating glomerular filtration rate from serum creatinine and cystatin C. N Engl J Med 2012;367:20-9

28. Spallone V, Morganti R, D'Amato C, Greco C, Cacciotti L, Marfia GA. Validation of DN4 as a screening tool for neuropathic pain in painful diabetic polyneuropathy. Diabet Med 2012;29:578-85

29. Toth C, Lander J, Wiebe S. The prevalence and impact of chronic pain with neuropathic pain symptoms in the general population. Pain Med 2009;10:918-29

30. Bouhassira D, Attal $\mathrm{N}$, Alchaar $\mathrm{H}$, et al. Comparison of pain syndromes associated with nervous or somatic lesions and development of a new neuropathic pain diagnostic questionnaire (DN4). Pain 2005;114:29-36

31. Hermeling E, Reesink KD, Kornmann LM, Reneman RS, Hoeks AP. The dicrotic notch as alternative time-reference point to measure local pulse wave velocity in the carotid artery by means of ultrasonography. J Hypertens 2009;27:2028-35

32. Willekes C, Hoeks AP, Bots ML, Brands PJ, Willigers JM, Reneman RS. Evaluation of off-line automated intima-media thickness detection of the common carotid artery based on M-line signal processing. Ultrasound in medicine \& biology 1999;25:57-64

33. De Bie SE. Standaardvragen 1987: Voorstellen voor uniformering van vraagstellingen naar achtergrondkenmerken en interviews. Leiden, Leiden University Press, 1987

34. Sheehan DV, Lecrubier $\mathrm{Y}$, Sheehan $\mathrm{KH}$, et al. The Mini-International Neuropsychiatric Interview (M.I.N.I.): the development and validation of a structured diagnostic psychiatric interview for DSM-IV and ICD-10. J Clin Psychiatry 1998;59 Suppl 20:22-33;quiz 4-57

35. Stringhini S, Sabia S, Shipley M, et al. Association of socioeconomic position with health behaviors and mortality. JAMA 2010;303:1159-66

36. Jacobs $M$, van Greevenbroek MM, van der Kallen $\mathrm{CJ}$, et al. The association between the metabolic syndrome and peripheral, but not coronary, artery disease is partly mediated by endothelial dysfunction: the CODAM study. Eur J Clin Invest 2011;41:167-75

37. Laramee AS, Morris N, Littenberg B. Relationship of literacy and heart failure in adults with diabetes. BMC Health Serv Res 2007; 7:98

38. Tesfaye S, Chaturvedi N, Eaton SE, et al. Vascular risk factors and diabetic neuropathy. N Engl J Med 2005;352:341-50

39. van Hateren KJ, Drion I, Kleefstra N, et al. A prospective observational study of quality of diabetes care in a shared care setting: trends and age differences (ZODIAC-19). BMJ Open 2012;2

40. Landelijke Organisatie voor Ketenzorg (LOK). Transparante ketenzorg Diabetes Mellitus, COPD, en VRM. Rapportage zorggroepen over 2012. Op weg naar genuanceerde rapportage van zorg. September 2013

41. Parker RM, Baker DW, Williams MV, Nurss JR. The test of functional health literacy in adults: a new instrument for measuring patients' literacy skills. J Gen Intern Med 1995;10:537-41

42. Davis TC, Long SW, Jackson $\mathrm{RH}$, et al. Rapid estimate of adult literacy in medicine: a shortened screening instrument. Fam Med 1993;25:391-5

43. Osborn CY, Cavanaugh K, Wallston KA, Rothman RL. Self-efficacy links health literacy and numeracy to glycemic control. J Health Commun 2010;15 Suppl 2:146-58

44. Nutbeam D. Health promotion glossary. Health Promot Int 1998;13:349-64

45. Schillinger D, Barton LR, Karter AJ, Wang F, Adler N. Does literacy mediate the relationship between education and health outcomes? A study of a low-income population with diabetes. Public Health Rep 2006;121:245-54

46. Deary IJ, Spinath FM, Bates TC. Genetics of intelligence. Eur J Hum Genet 2006;14:690-700

47. Deary IJ, Johnson W. Intelligence and education: causal perceptions drive analytic processes and therefore conclusions. Int J Epidemiol 2010;39:1362-9 
48. Brown AF, Ettner SL, Piette J, et al. Socioeconomic position and health among persons with diabetes mellitus: a conceptual framework and review of the literature. Epidemiol Rev 2004;26:63-77 


\section{Chapter 7}

GENERAL DISCUSSION 
Chapter 7 


\section{General discussion}

A growing body of evidence shows that type 2 diabetes is associated with an increased risk of cognitive impairment and dementia. Studying risk factors for cognitive decline may yield modifiable risk factors for dementia and cognitive impairment. Therefore, the central topic of the studies in this thesis was to examine the relationship of type 2 diabetes, exposure to associated vascular risk factors, and body composition with cognitive functioning. In addition, one study was focused on the likelihood of diabetic complications, which may be increased in patients in whom the skills required to understand and act on health-related instructions are inadequate. In this particular study, we did not use cognitive performance as an outcome, but instead we used diabetic complications and walking speed as outcomes and a cognitive variable, i.e. verbal intelligence, as a determinant.

The current chapter first summarizes the main findings of this thesis and relates these to each other and the current literature. Next, possible limitations, particularly methodological considerations, are discussed, followed by implications for clinical practice and research. At the end of this chapter a general conclusion is presented.

\section{Main findings}

\section{Cognitive decline in type 2 diabetes}

Previous studies have shown that type 2 diabetes has been associated with an increased risk of dementia. However, studies on cognitive decline in type 2 diabetes have shown inconsistent results. ${ }^{1-4}$ In Chapter 2, patients with type 2 diabetes showed accelerated cognitive decline compared with individuals without diabetes, particularly in information processing speed and concept shifting (executive function). Interestingly, we found that patients who developed diabetes during the study showed signs of early decline in information processing speed only. In line with previous research, ${ }^{5}$ our results suggest that disease duration is an important factor in diabetes-related cognitive decline. Although recently diagnosed diabetic patients may be at risk for accelerated cognitive decline, it seems that in the early phase of the disease few cognitive domains are affected and cognitive decline is rather small.

\section{Associations of vascular risk factors and body composition with cognitive functions}

\section{Advanced glycation end-products (AGEs) and cognitive functions}

One important mechanism that is involved in the development of vascular complications and is associated with both aging and diabetes, is the accumulation of advanced glycation end-products (AGEs). In addition, AGEs are found in amyloid plaques and neurofibrillary tangles in the brains of patients with Alzheimer's disease 
$(A D)^{6}$ and thus may also play a role in the pathophysiology of AD. Therefore, accumulation of AGEs may be related to the development of cognitive decline. Indeed, the results in Chapter 3 show that skin autofluorescence (SAF), a marker for tissue AGE accumulation, is associated with cognitive functions, particularly memory. Plasma AGEs, however, were not associated with cognitive functions. In addition, this chapter shows that AGEs may exert their effect on cognitive performance through vascular risk factors and/or complications. These results suggest that the accumulation of tissue AGEs is involved in the development of cognitive decline, particularly memory decline, and possibly in part through the action of vascular disease/risk factors.

\section{Ambulatory blood pressure and cognitive functions}

Hypertension is common in individuals with type 2 diabetes and previous studies have shown interactive effects of hypertension and diabetes on cognitive decline and dementia., In Chapter 4, we hypothesized that associations of 24-hour blood pressure and abnormal dipping with cognitive performance are stronger in individuals with type 2 diabetes compared with those without diabetes. The results show that both high and low diastolic 24-hour blood pressure were indeed associated with a worse performance on tasks for information processing speed and memory in individuals with diabetes, but not in those without. No clear patterns were found in the associations of abnormal dipping with cognitive performance in both individuals with and without diabetes. This study therefore suggests that both low and high (diastolic) blood pressure may increase the risk of cognitive impairment in individuals with type 2 diabetes.

\section{Body composition and cognitive status at late life}

A high BMI in midlife has been associated with worse cognitive performance and a higher risk of dementia, ${ }^{9,10}$ while in late life a higher BMI has been associated with a decreased risk of dementia. ${ }^{10} \mathrm{~A}$ decrease in weight and BMI may occur several years before a diagnosis of dementia and may be caused by neurodegenerative processes in the brain. ${ }^{11}$ In Chapter 5, results show that a higher amount of subcutaneous fat, a major source of leptin and estrogens, was associated with a decreased likelihood of dementia in older women, while a higher amount of muscle tissue was associated with a decreased likelihood of dementia in both older men and women. Furthermore, in women with a low BMI at midlife these associations were strongest. These findings suggest that changes in body composition associated with dementia may be mainly attributed to subcutaneous fat and muscle tissue.

\section{Low verbal intelligence, diabetic complications, and walking speed}

To understand diabetes, its consequences, and how to cope with it in an effective way, adequate health literacy is necessary. However, inadequate health literacy is 
common in diabetic patients. ${ }^{12}$ Results of Chapter 6 show that verbal intelligence, a measure of premorbid IQ and probably a core constituent of health literacy, is indeed associated with diabetic complications and walking speed, although no associations were found with important determinants of diabetic complications, including glycemic, blood pressure, or lipid control. Interestingly, associations of verbal intelligence with diabetic complications and slower walking speed, a measure of physical functioning and a strong predictor of health status, ${ }^{13,14}$ seemed to be largely explained by educational level. Our results suggest that diabetic patients with a low verbal intelligence may have an increased risk of developing diabetic complications and may show reduced physical function compared with patients with a higher verbal intelligence.

\section{Putting the pieces together}

\section{Risk factors for cognitive decline}

After studying several risk factors for cognitive impairment and decline it is important to put these factors together and try to understand how these factors either alone or together may have their impact on cognitive decline. Therefore, the associations examined in this thesis are summarized and connected with each other in Figure 7.1. This figure depicts potential mechanisms that can explain associations of diabetes, its associated vascular risk factors, and body composition (adiposity) with cognitive decline. Variables within this figure all can affect brain reserve, i.e. the amount of intact brain volume, synapses, and connectivity between neurons. A reduction in brain reserve lowers the threshold for subsequent brain damage to produce cognitive deficits. $^{15}$

First of all, type 2 diabetes can reduce brain reserve and consequently accelerate cognitive decline, independent of other vascular risk factors. Several studies have shown that diabetes is associated with cerebral vascular lesions and atrophy, which may cause cognitive impairment. Diabetes has been associated with lacunar infarcts, ${ }^{16}$ less consistently with cerebral white matter lesions, ${ }^{17,18}$ and with cerebral microbleeds. ${ }^{19}$ Further, diabetes has been associated with brain atrophy, particularly in the hippocampus and amygdala ${ }^{20,21}$ which have been involved in the development of $A D$. These changes in the brain may contribute to cognitive impairment and indeed some studies have shown that infarcts and deep white matter lesions ${ }^{22}$ and/or brain atrophy ${ }^{22,23}$ may contribute to poorer cognitive performance in individuals with type 2 diabetes. Therefore, both cerebrovascular lesions and neurodegeneration may be involved in the development of cognitive impairment in type 2 diabetes. But how can diabetes lead to this cerebral damage? Mechanisms involved in the development of cerebral damage in individuals with type 2 diabetes include micro- and macrovascular disease, insulin resistance, and hyperglycemia. ${ }^{24}$ 


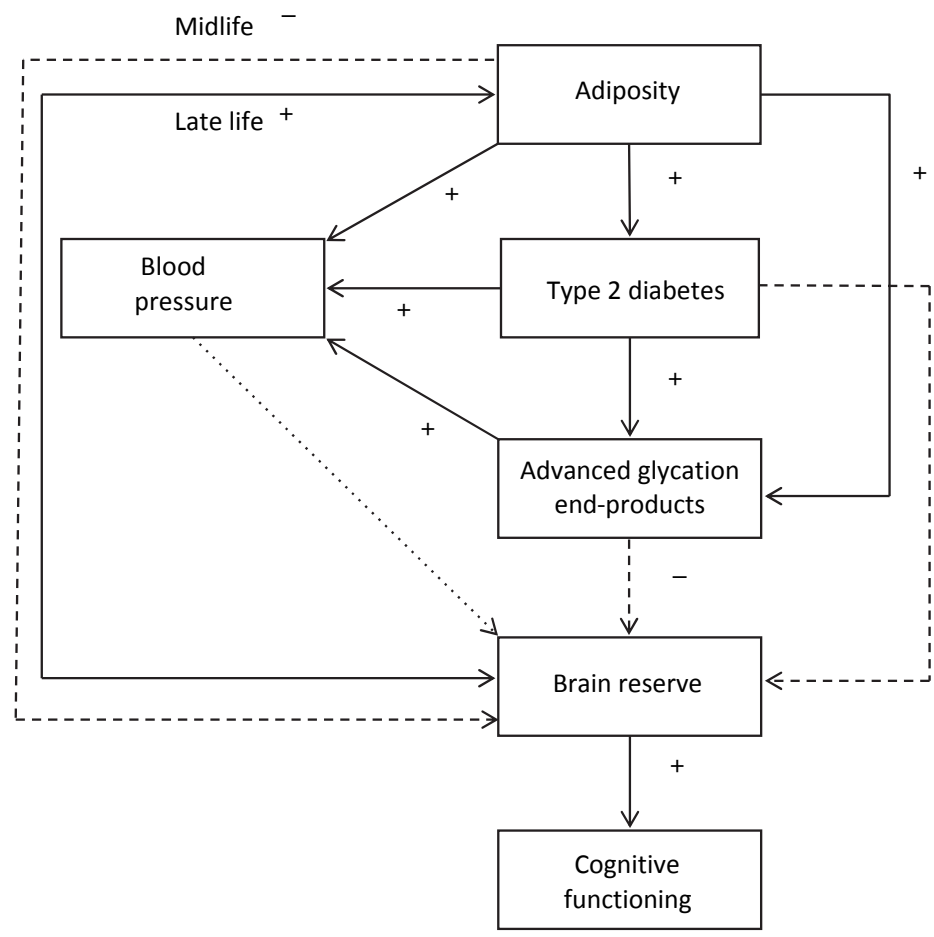

Figure 7.1 Potential mechanisms explaining the associations of diabetes and its related vascular risk factors with cognitive functioning. Solid lines indicate positive associations and dashed lines negative associations. The dotted line indicates an inverted U-shaped association. Arrows indicate the direction of the associations and double-headed arrows indicate that associations may be bi-directional.

First, diabetes increases the risk for ischemic stroke ${ }^{25}$ through development atherosclerosis ${ }^{26}$ and could thereby lead to accelerated cognitive decline. ${ }^{22}$ Further, chronic exposure to hyperglycemia has been associated with basement membrane thickening of the cerebral capillaries, which can lead to chronic or insidious ischemia of the brain. ${ }^{27}$ Second, insulin seems to compete with $\beta$-amyloid for insulin-degrading enzyme and high levels of insulin, which are associated with diabetes, may increase the levels of $\beta$-amyloid in the brain. ${ }^{28}$ This diversion of insulin-degrading enzyme from the metabolism of $\beta$-amyloid to the metabolism of insulin may cause a deficiency of the enzyme and can lead to increased accumulation of $\beta$-amyloid in the brain. ${ }^{28}$ Last, but not least, hyperglycemia can lead to an increased accumulation of AGEs.

Accumulation of AGEs in the brain may reduce brain reserve and increase the risk of cognitive impairment through modification of $\beta$-amyloid, which exacerbates the toxicity of this peptide. ${ }^{29}$ In addition, AGEs in the brain could lead to cognitive impairment by increasing inflammation, oxidative stress and subsequently neuronal 
dysfunction. ${ }^{29}$ As suggested by Chapter 3, AGEs may decrease brain reserve through its associations with vascular risk factors/complications, such as hypertension and cardiovascular disease, which (previously) have been associated with accumulation of AGEs. ${ }^{30}$ These factors can also contribute to development of cognitive impairment. Accumulation of AGEs may reduce brain reserve and thereby increase the risk of cognitive impairment in individuals with and without diabetes. However, AGEs accumulation may also mediate the association between diabetes and cognitive impairment. One previous study has shown that the association of diabetes with lower grey matter volume was partially mediated $(20 \%)$ by skin autofluorescence, a marker for tissue AGE accumulation, ${ }^{31}$ which suggests that AGE accumulation is one of many pathways that contribute to brain atrophy and subsequently cognitive impairment in individuals with type 2 diabetes.

Type 2 diabetes is strongly associated with high blood pressure, which has also been associated with brain atrophy ${ }^{32}$ and cognitive decline, ${ }^{33}$ possibly through the development of cerebral small vessel disease. ${ }^{32,34}$ Furthermore, diabetes and hypertension have been shown to exert interactive effects on brain atrophy and cognitive decline. ${ }^{7,18}$ Low blood pressure may also cause a reduction in brain reserve by hypoperfusion of the brain and thereby to atrophy. Hypoperfusion can occur in the presence of arterial stiffness and atherosclerosis, impaired cerebral autoregulation, and orthostatic hyportension. These conditions ${ }^{35-37}$ as well as cerebral small vessel disease ${ }^{38}$ have also been associated with diabetes, which may explain why we found a $\mathrm{U}$-shaped association between diastolic blood pressure and cognitive functions in individuals with diabetes, but not in those without (Chapter 4).

Adiposity increases the risk of developing type 2 diabetes, may increase tissue AGE accumulation, ${ }^{39}$ and has been associated with cognitive impairment, particularly midlife adiposity. ${ }^{9,10}$ However, at late life, the association of adiposity with cognitive impairment seems to change. ${ }^{9,10}$ A decrease in weight and BMI can occur several years before a diagnosis of dementia ${ }^{11}$ and may be caused by neurodegenerative processes in the brain. In Chapter 5, higher amounts of subcutaneous fat, and also muscle tissue, were associated with a decreased likelihood of dementia. Several factors that are directly related to neuronal dysfunction and neurodegeneration could lead to unintended weight loss in patients with dementia. These factors include cognitive and psychiatric problems, altered olfaction and gustation, and pathology of energy homeostatic centers in the brain, particularly the hypothalamus and autonomic centers. ${ }^{40}$ Dementia patients may forget to eat due to their memory problems, or may be less motivated to eat due to loss of initiative and/or due to loss in sense of smell that can accompany dementia. ${ }^{41,42}$ However, loss of fat, particularly subcutaneous fat, may also contribute to a (further) reduction in brain reserve and subsequently cognitive decline, particularly in women. Mechanisms underlying this association may include decreased levels of leptin and estrogens, which are produced by subcutaneous fat and have been shown to have positive effects on the brain and cognitive functions. ${ }^{43,44}$ The association between late-life adiposity and brain reserve may 
therefore be bi-directional. Furthermore, loss of muscle tissue (not shown in Figure 7.1) may be linked to cognitive decline through the production of inflammatory factors, such as interleukin- $9,{ }^{45,46}$ which has been associated with both muscle loss and dementia.

The effects of diabetes on cognitive decline in individuals without dementia may be distinct from the decline seen in patients with dementia and subtle cognitive changes in diabetic patients seem to develop slowly over time. ${ }^{47}$ Furthermore, as previously mentioned, effects of diabetes on cognition may be most pronounced after the age of $60 .{ }^{48}$ Although effects of diabetes on cognitive decline may be subtle and slow, diabetes seems to reduce brain reserve, like AGE accumulation, low and high blood pressure, and possibly, to some extent, loss of fat, and may lower the threshold for neuropathology to result in accelerated cognitive decline. In addition, vascular risk factors may also directly interact with neuropathology, for example by increasing levels or toxicity of $\beta$-amyloid in the brain.

Vascular risk factors often coexist and can cluster in the "metabolic syndrome" which has also been associated with accelerated cognitive decline. ${ }^{49}$ Therefore it is likely that these risk factors affect the brain by multiple pathways, as shown in Figure 7.1. Furthermore, the presence of multiple vascular risk factors may lead to additive or even synergistic effects on cognitive decline. ${ }^{50,51}$

\section{Verbal intelligence, educational level and diabetic complications}

In Figure 7.2, the association of verbal intelligence with diabetic complications is depicted. Verbal intelligence is suggested to be a core constituent of health literacy and may therefore determine the degree of health literacy of diabetic patients. Verbal intelligence is also an important predictor of educational level, ${ }^{52}$ which explained to a large extent the associations of verbal intelligence with cardiovascular disease, neuropathic pain, and walking speed (Chapter 6). Furthermore, educational level can also influence health literacy. ${ }^{53}$ In Figure 7.2, we propose that educational level is an important mediator in the relationship between verbal intelligence and diabetic complications, as verbal intelligence has been shown to be highly heritable ${ }^{54}$ and to be a predictor of educational level. ${ }^{52}$ Individuals with a lower verbal intelligence are more likely to receive less formal education and may therefore not develop adequate health literacy skills. It must be noted, however, that educational level still can have some influence verbal intelligence ${ }^{55}$ and that some individuals who had a low verbal intelligence in childhood, may have followed many years of formal education and may thereby have improved both their verbal intelligence and health literacy skills. 


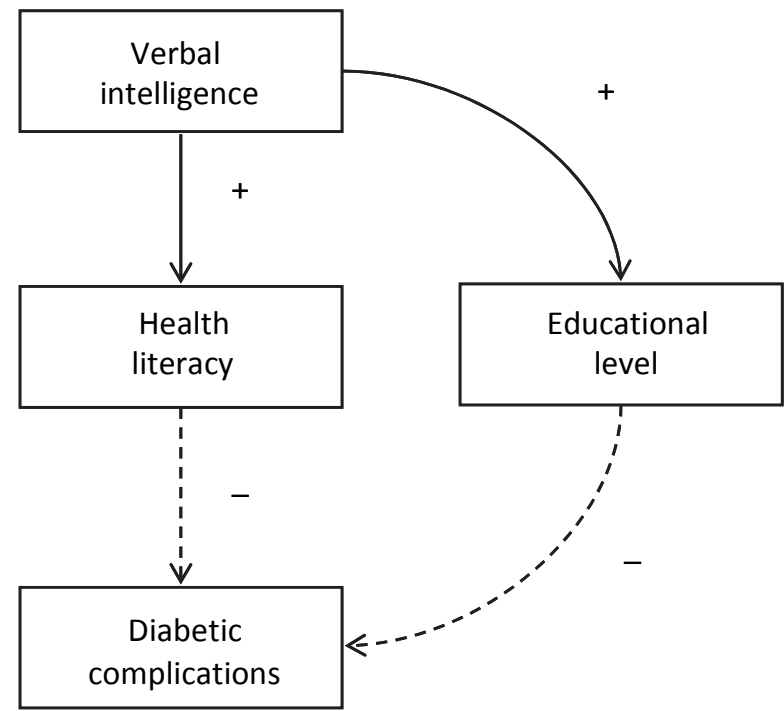

Figure 7.2 Potential mechanisms explaining the association between verbal intelligence and diabetic complications. Solid lines indicate positive associations and dashed lines negative associations. Arrows indicate the direction of the associations.

\section{Methodological considerations ${ }^{a}$}

\section{Cross-sectional vs. longitudinal designs}

In Chapters 3-6, we used a cross-sectional design to test our hypotheses. One advantage of a cross-sectional study is that there is no loss to follow-up. Loss to follow-up can be a problem in longitudinal studies, because it can induce bias. One drawback of a cross-sectional study design is that causality cannot be inferred. Longitudinal designs are necessary to make more firm conclusions about causality. Also, failing to find cross-sectional associations does not mean that longitudinal associations do not exist. The effect of vascular risk factors on cognition may only become apparent when change in cognition over time is examined. During a follow-up period, individuals can show cognitive aging and can develop cognitive impairment, processes that cannot be assessed by cross-sectional studies. Some individuals may show cognitive performance that is not different from the average performance in the study sample, although their performance has declined when compared with their performance several years before. In Chapter 3, for example, some modest associations were found between a marker for tissue AGEs and cognitive

a Published in part in Spauwen PJ \& Stehouwer CD. Cognitive decline in type 2 diabetes. Lancet Diabetes Endocrinol. 2014;2:188-9 
performance, which became non-significant after adjustment for potential confounders/mediators. One reason for not finding stronger associations in this study may have been the use of a cross-sectional design. In line with this, one previous study found no significant cross-sectional associations between plasma AGEs and cognitive performance, but did find associations between AGEs and cognitive change over time. $^{56}$ The issue of cross-sectional data also plays a role in Chapters $5 \& 6$. In Chapter 5, no conclusion could be drawn about whether dementia pathology causes changes in body composition or whether changes in body composition contribute to the development cognitive impairment. In Chapter 6, we found associations of verbal intelligence with diabetic complications, but not with intermediate determinants of these complications. Nevertheless, cross-sectional studies remain valuable, because they may point towards an association between two variables, regardless of the direction of this relationship. In addition, results of cross-sectional studies can be used as an important step towards formulating hypotheses for longitudinal research.

\section{Follow-up duration}

Ideal study designs are (longitudinal) designs with a long follow-up duration. Previous cross-sectional research has suggested that individuals with diabetes show relatively subtle decrements in cognitive functioning, with moderate effect sizes of about -0.3 to $0.5 \mathrm{SD}^{57}$ In addition, longitudinal studies with an average follow-up duration of 5 years have reported decline that exceeded the effects of normal ageing by a factor of 1.5-2.0. ${ }^{47}$ However, some studies have shown rates of decline in diabetic patients that are within the range of normal cognitive aging. ${ }^{1,3}$ In addition, the effect of newly diagnosed diabetes on cognitive functions seems to be small. In Chapter 2, the change in information processing speed over a long period of 12 years in individuals with baseline diabetes relative to those without baseline diabetes was -7.6 points, while this was -1.6 points for individuals with incident diabetes relative to those without diabetes during the whole study. Other studies also have shown that cognitive decline in newly diagnosed diabetic patients is small and often not statistically different from those without diabetes. ${ }^{58-60}$

It has been suggested that diabetes has a clinically relevant effect on cognitive functions when the brain undergoes neurodegenerative changes due to ageing, commonly occurring at ages older than $65 .{ }^{48}$ One previous study has shown that diabetes in middle-aged individuals accelerated cognitive decline (1.25 to 1.45 times faster than in normal ageing), ${ }^{59}$ although the rate of decline was less than in previous studies (1.5-2.5). ${ }^{47}$ Because most previous studies were performed in older individuals with diabetes, this suggests that the effect of diabetes on cognition may accelerate with age. In addition, longer diabetes duration may also accelerate cognitive decline. ${ }^{5,59}$ All these factors may indicate that a long follow-up duration is required to find (profound) effects of both baseline and incident diabetes on cognitive decline. A reason that could explain the strong effects (found in Chapter 2) of diabetes on cognitive change over time, may be the long follow-up duration ( $>5$ years) and the 
mean age of the diabetes sample (>65 years). In order to know which individuals with diabetes, including new-onset diabetes, will show accelerated cognitive decline, a long follow-up period of probably at least 10 years is needed.

Age is also an important factor in the association of obesity and blood pressure with cognitive decline. As mentioned previously, the effect of obesity on cognitive decline seems to differ with age. In addition, this age-dependent effect has also been shown for blood pressure. While a high blood pressure at midlife seems to accelerate cognitive decline and increase the risk for $A D$ and dementia, a low blood pressure in later life has been associated with a higher risk of dementia and AD. ${ }^{61,62}$ The reversed association in late life may be explained by cerebral hypoperfusion, as a result of atherosclerosis and arterial stiffening, or by pathological brain changes due to dementia which may influence blood pressure regulation. ${ }^{61,62}$ As associations of some vascular risk factors with cognitive decline may change over time, it is important to examine these associations over a long period of time with assessments taken in midlife and in late life.

\section{Cognitive markers vs. biomarkers}

The studies in this manuscript have used cognitive performance as an outcome measure. One advantage of using cognitive measures to indicate brain changes is that these measures are non-invasive. In addition, cognitive performance has been shown to be a good predictor of the development of dementia. ${ }^{63,64}$ However, neurodegenerative changes do not always lead to lower cognitive performance, especially not in an early stage of dementia. It has been suggested that biomarkers in cerebrospinal fluid (CSF) are the most informative in the very early prodromal stages, ${ }^{63,65}$ which may be important stages to start interventions in order to delay or prevent (further) cognitive decline. In addition, brain volumetric biomarkers may also be more informative than neuropsychological test results, because a small reduction in brain volume may not yet be sufficient to produce a cognitive deficit ${ }^{15}$ and because the process of brain atrophy seems to occur at an earlier stage in the dementia development than cognitive decline. ${ }^{65}$

Although CSF and brain volumetric biomarkers seem to become abnormal before cognition starts to decline, previous studies have shown that neuropsychological tests were the best individual predictors of dementia in individuals with mild cognitive impairment $(\mathrm{MCl})$ compared with other markers, including hippocampal volume and CSF markers of neuropathological changes associated with AD. However, a combination of these markers gave the best prediction. ${ }^{63,64}$ Furthermore, it has been suggested that in individuals with vascular disease the lag time between pathological changes in the brain associated with $A D$ and cognitive decline becomes shorter. ${ }^{65}$ This again may indicate that decline in cognitive performance is an important predictor of dementia, especially in individuals with vascular disease in whom the threshold for brain damage sufficient to produce cognitive deficits may be lower (reduced brain reserve). 
Cognitive markers, which are less expensive and not invasive, will remain to be important outcome measures, because cognitive problems are eventually the final outcome. Particularly in large longitudinal studies, it is often not feasible to measure biomarkers and acquire magnetic resonance imaging (MRI) data. Ideally, cognitive markers are used together with CSF markers and MRI data. MRI data should include volumetric measures of the frontal, medial and temporal lobes, which have been associated with the development of dementia, ${ }^{65}$ and measures of white-matter hyperintensities, lacunar infarcts, and cerebral microbleeds which have been associated with vascular risk factors and cognitive decline.

\section{Neuropsychological assessment}

When using cognitive performance as an outcome, it is important to select tests that are sensitive to vascular risk factor exposure, such as diabetes. ${ }^{66}$ In people with type 2 diabetes, cognitive decrements are most consistently reported for measures of verbal memory and information processing speed, ${ }^{67}$ although in older individuals with diabetes, decrements in executive functioning can also be seen. ${ }^{58}$ Furthermore, informationprocessing speed seemed to be most sensitive to the effects of new-onset diabetes, as shown in Chapter 2 and in a previous cross-sectional study. ${ }^{68}$ Choosing tests that are not sensitive enough may lead to finding weak or no associations. Therefore, tests assessing domains of information processing speed, memory, and executive functions should always be used when examining effects of diabetes and diabetes-associated vascular risk factors on cognitive decline. This battery of neuropsychological tests may be expanded by tests for attention/concentration and motor function, as a recent meta-analysis showed small to moderate effect sizes for these domains in individuals with diabetes relative to those without. ${ }^{69}$ However, attention/concentrations tests seemed to be less sensitive to effects of diabetes than domains of processing speed, memory, and executive function, and the association of diabetes with motor function needs to be further elucidated, since peripheral neuropathy may play a role. ${ }^{69}$

\section{Characteristics of the databases}

In this thesis, datasets from several studies were used; each with its own focus and specialization. Consequently, each dataset contains different characteristics with its own strengths and limitations, which will be outlined in the next paragraphs.

Two major advantages of The Maastricht Aging Study ${ }^{70}$ include the comprehensive neuropsychological assessment and the long follow-up duration, which permits drawing a conclusion about the long-term effects of vascular risk factors, in our study type 2 diabetes, on cognitive change over time. In addition, this dataset made it possible to examine the effect of incident diabetes, measured at two time points, on cognitive change over time. This is particularly important because it increases our knowledge on early effects of diabetes on cognition. However, as this 
study was mainly focused on cognitive aging and cognitive assessment, measurement of diabetes status was not based on blood samples, but on self-report and medication use only. This may have led to an underestimation of the effect of type 2 diabetes on cognitive decline, as some individuals with diabetes may have been wrongly assigned to the nondiabetic group. In addition, other potentially important biomarkers, including glycated hemoglobin A1c (HbA1c) levels, were not measured. Furthermore, the sample sizes of the prevalent and incident diabetes groups were relatively small. Future studies with a comparable follow-up duration, but with larger sample sizes and blood test data, may lead to more precise estimates of the effect of type 2 diabetes on cognitive change over time. The inclusion of biomarkers, like HbA1c levels, may increase our understanding of mechanisms underlying this effect.

The Maastricht Study includes multiple elaborate measurements of vascular risk factors and assessment of most important cognitive domains. ${ }^{71}$ Elaborate measures include, amongst others, plasma AGEs, skin autofluorescence, ambulatory blood pressure measures, 24-hour urine collections, and carotid intima media thickness. Therefore, The Maastricht Study is expected to become one of the most extensive phenotyping studies in both the general population and diabetes participants worldwide. Furthermore, diabetes status is based on a 2-hour oral glucose tolerance test, which is the golden standard to diagnose type 2 diabetes. Unfortunately, only cross-sectional data of The Maastricht Study were available at this stage. Consequently, conclusions about causality have been made with some caution.

Cross-sectional data from the AGES-Reykjavik study ${ }^{72}$ were used to examine associations of body composition with cognitive status. Some factors that make this study unique are the large sample size, the old population (aged 75+ years), elaborate measurements of vascular risk factors, cognitive functions, computed tomography (CT) measures of body composition, and midlife measurements including BMI and weight. Furthermore, this study enabled a clinical diagnosis of $\mathrm{MCl}$ or dementia, which was based on consensus of a geriatrician, a neurologist, a neuropsychologist, and a neuroradiologist. Longitudinal data are now available and although conclusions about the direction of the relationship between body composition and dementia cannot be drawn in this thesis, this will probably be possible in the near future. Despite the fact that longitudinal data are required to examine associations with change over time, loss-to-follow-up may become an issue, especially in this older population.

\section{Recommendations}

\section{Clinical implications}

The clinical implications of some findings of this thesis involve the identification of certain vascular risk factors, especially type 2 diabetes, for cognitive decline. It is important for clinicians to get insight into the vascular risk profile, including diabetes status and blood pressure, and to monitor these factors as well as cognitive 
performance. Better control of diabetes and blood pressure may contribute to less cognitive decline.

Another important factor in the clinical practice with regard to cognitive impairment and dementia is age. In late life, more subcutaneous fat (and muscle tissue) seems to be associated with a decreased likelihood of dementia, particularly in women. Clinicians should be aware that, in old age, loss of fat and muscle tissue might be associated with degeneration of the brain and decline in cognitive functions. To decrease the potential negative influence of fat and muscle loss on the brain and to prevent severe consequences that may accompany weight loss, such as increased mortality, morbidity, and progressive disability in older individuals, ${ }^{73}$ it seems important to monitor weight changes and to develop programs aimed at maintaining weight and body composition, like dietary interventions and muscle strength training.

In addition to cognitive impairment and dementia, micro- and macrovascular disease are also severe complications of diabetes. The findings in this thesis stress that verbal intelligence and educational level may contribute to the likelihood of these complications. Therefore, clinicians should ask their patients about their educational level, because this may indicate their level of health literacy. A low educational level may indicate that the patient has inadequate health literacy skills and is at increased risk to develop diabetic complications. Several interventions could help to improve health literacy skills and self-management according to the World Health Organization report on Health Literacy ${ }^{74}$ and may therefore decrease the risk of complications in diabetic patients. First, as educational level seems to be an important predictor of health literacy skills and diabetic complications, it may be important to encourage people to be lifelong learners, for example through daily reading or learning computer skills, in order to facilitate the development and maintenance of health literacy skills. Second, health care providers should use plain language, use clear and simple written and spoken language, and should check whether the patient has understood their instructions, especially, but not exclusively, when the patient is low educated. To help health care providers tailor their instructions, a universal medication schedule has been promoted as a way to standardize these instructions. An example of such written instruction is "take two pills in the morning and two pills in the evening" in stead of "take two tablets twice daily." Using simple and standardized instructions could improve medication adherence in diabetic patients. Third, chronic disease selfmanagement programs may improve self-care behaviors and decrease the risk of diabetic complications and are now being implemented in several European countries, including the Netherlands.

\section{Scientific implications, future directions}

The findings of this thesis have several implications for future studies examining (vascular) risk factors and cognitive decline. Ideally, these studies should 1) be longitudinal, 2) have long follow-up durations, 3) include assessments in both midlife and late life, 4) include diagnoses of prevalent and incident dementia to increase our 
understanding of the cognitive trajectories in individuals who will develop dementia and to understand the influence of vascular risk factors on these trajectories.

Such studies should definitely examine the effect of AGE accumulation on change in multiple cognitive functions over time, as this has not been adequately addressed. In addition, studies should examine whether skin autofluorescence is a predictor for cognitive impairment and dementia. This could, for example, indicate whether the use of a skin autofluorescence reader, as a non-invasive measure of tissue AGE accumulation, has predictive value in clinical settings. Further, the relationship between body composition and cognitive decline needs to be further elucidated and future studies should consider the 4 points mentioned above. Such studies could increase our understanding about the direction of the association between body composition and cognitive impairment.

The ultimate goal of studying modifiable risk factors for cognitive decline and dementia will be to delay or prevent the development of dementia. Recent studies have suggested that the incidence of dementia is decreasing, possibly as a result of the improved control of vascular risk factors and cardiovascular disease. ${ }^{75,76}$ Therefore, studies are needed that examine how these risk factors can be modified and whether better control of these risk factors could indeed at least significantly delay the development of dementia. Several large randomized control trials (RCT) have examined the effect of treatment of vascular risk factors on dementia and cognitive decline, although cognition was used as a secondary outcome in these studies ${ }^{77}$ and most of these studies have focused on treatment of one single vascular risk factor. The treatment of hypertension seemed to have the most promising effect. $^{78,79}$ However, most studies failed to find an effect on dementia and cognitive decline, which may be due to the study design. For example, follow-up durations may have been too short and the age of the sample may have been too high. As mentioned earlier, in late life, vascular risk factors, like hypertension and obesity, may not increase the risk for dementia, and lowering blood pressure and losing weight may not have an effect on the risk of dementia. Some large RCTs with relatively long follow-up durations (6-7 years) and with a multifactorial approach are ongoing and may reveal that intensive treatment of vascular risk factors can slow down or prevent cognitive decline. ${ }^{77}$ However, these trials are focused on elderly, which may not be the best population to target vascular risk factors in order to prevent cognitive decline or dementia. Treatment effects may therefore not be found or may not be as large as in middle-aged individuals.

The Innovative, Midlife intervention for Dementia Deterrence (In-MINDD) is a new study that will develop and validate online tools to help physicians to assess if an individual's lifestyle supports long-term brain health. ${ }^{80}$ This study will generate personal strategies for adopting a brain healthy lifestyle by stimulating change in risk factors for dementia, including smoking, blood pressure, and cholesterol. Studies, like In-MINDD, which is currently being conducted, possibly increase our understanding about how to delay or prevent dementia. One of the main advantages of In-MINDD is 
the multifactorial approach. Multiple factors can increase the risk of cognitive decline and dementia and should therefore all be considered when trying to increase brain health. Dementia is a multi-causal syndrome and should therefore be treated as such.

Last, in order to decrease the risk of diabetic complications longitudinal studies on the association between on the one hand, verbal intelligence, education level, and health literacy, and on the other hand development of diabetic complications, are warranted. Such studies may increase our insight into the causal relationship between a decreased capacity to understand instructions for disease management and the increased likelihood of diabetic complications. These studies should also include diabetes-specific measures of health literacy and numeracy to understand which specific capabilities are decreased and should be addressed in order to increase diabetes knowledge, motivation, and medication adherence and to eventually decrease the risk of diabetic complications.

\section{Conclusions}

In this thesis we have examined risk factors for cognitive decline and dementia. We conclude that vascular risk factors are associated with cognitive functions and may be important targets to delay or prevent cognitive decline. Vascular risk factors are interrelated, their effect on cognitive decline may change over time, and they may lead to cognitive impairment through multiple mechanisms. All these aspects should be considered when interpreting results and making conclusions, especially the association of body composition with cognitive status in late life does not seem to be straightforward. In addition, we have linked verbal intelligence to the presence of diabetic complications and walking speed, a measure of physical functioning and important predictor of health status, in individuals with type 2 diabetes. Low verbal intelligence and low educational level seem to contribute to a higher likelihood of diabetic complications, which indicates that it is important for clinicians to assess the educational level of their diabetic patients and to provide information at a level of complexity that can be understood by these patients. 


\section{References}

1. Fischer AL, de Frias CM, Yeung SE, Dixon RA. Short-term longitudinal trends in cognitive performance in older adults with type 2 diabetes. J Clin Exp Neuropsychol 2009;31:809-22

2. Yaffe K, Blackwell T, Kanaya AM, Davidowitz N, Barrett-Connor E, Krueger K. Diabetes, impaired fasting glucose, and development of cognitive impairment in older women. Neurology 2004;63: 658-63

3. van den Berg E, Reijmer YD, de Bresser J, Kessels RP, Kappelle LJ, Biessels GJ. A 4 year follow-up study of cognitive functioning in patients with type 2 diabetes mellitus. Diabetologia;53:58-65

4. Wessels AM, Lane KA, Gao S, Hall KS, Unverzagt FW, Hendrie HC. Diabetes and cognitive decline in elderly African Americans: a 15-year follow-up study. Alzheimers Dement 2011;7:418-24

5. Okereke $\mathrm{OI}$, Kang JH, Cook NR, et al. Type 2 diabetes mellitus and cognitive decline in two large cohorts of community-dwelling older adults. J Am Geriatr Soc 2008;56:1028-36

6. Valente T, Gella A, Fernandez-Busquets X, Unzeta M, Durany N. Immunohistochemical analysis of human brain suggests pathological synergism of Alzheimer's disease and diabetes mellitus. Neurobiol Dis 2010;37:67-76

7. Hassing LB, Hofer SM, Nilsson SE, et al. Comorbid type 2 diabetes mellitus and hypertension exacerbates cognitive decline: evidence from a longitudinal study. Age Ageing 2004;33:355-61

8. Xu WL, Qiu CX, Wahlin A, Winblad B, Fratiglioni L. Diabetes mellitus and risk of dementia in the Kungsholmen project: a 6-year follow-up study. Neurology 2004;63:1181-6

9. Cournot M, Marquie JC, Ansiau D, et al. Relation between body mass index and cognitive function in healthy middle-aged men and women. Neurology 2006;67:1208-14

10. Fitzpatrick AL, Kuller $\mathrm{LH}$, Lopez $\mathrm{OL}$, et al. Midlife and late-life obesity and the risk of dementia: cardiovascular health study. Arch Neurol 2009;66:336-42

11. Stewart R, Masaki K, Xue QL, et al. A 32-year prospective study of change in body weight and incident dementia: the Honolulu-Asia Aging Study. Arch Neurol 2005;62:55-60

12. Schillinger $D$, Grumbach $K$, Piette J, et al. Association of health literacy with diabetes outcomes. JAMA 2002;288:475-82

13. Jylha M, Guralnik JM, Balfour J, Fried LP. Walking difficulty, walking speed, and age as predictors of self-rated health: the women's health and aging study. J Gerontol A Biol Sci Med Sci 2001;56:M609-17

14. Studenski S, Perera S, Wallace D, et al. Physical performance measures in the clinical setting. J Am Geriatr Soc 2003;51:314-22

15. Stern Y. What is cognitive reserve? Theory and research application of the reserve concept. J Int Neuropsychol Soc 2002;8:448-60

16. Longstreth WT, Jr., Bernick C, Manolio TA, Bryan N, Jungreis CA, Price TR. Lacunar infarcts defined by magnetic resonance imaging of 3660 elderly people: the Cardiovascular Health Study. Arch Neurol 1998;55:1217-25

17. Jongen C, van der Grond J, Kappelle LJ, et al. Automated measurement of brain and white matter lesion volume in type 2 diabetes mellitus. Diabetologia 2007;50:1509-16

18. Schmidt R, Launer $L$, Nilsson LG, et al. Magnetic resonance imaging of the brain in diabetes: the Cardiovascular Determinants of Dementia (CASCADE) Study. Diabetes 2004;53:687-92

19. Cordonnier C, Al-Shahi Salman R, Wardlaw J. Spontaneous brain microbleeds: systematic review, subgroup analyses and standards for study design and reporting. Brain 2007;130:1988-2003

20. Gold SM, Dziobek I, Sweat V, et al. Hippocampal damage and memory impairments as possible early brain complications of type 2 diabetes. Diabetologia 2007;50:711-9

21. den Heijer T, Vermeer SE, van Dijk EJ, et al. Type 2 diabetes and atrophy of medial temporal lobe structures on brain MRI. Diabetologia 2003;46:1604-10

22. Manschot SM, Brands AM, van der Grond J, et al. Brain magnetic resonance imaging correlates of impaired cognition in patients with type 2 diabetes. Diabetes 2006;55:1106-13

23. Moran C, Phan TG, Chen J, et al. Brain atrophy in type 2 diabetes: regional distribution and influence on cognition. Diabetes Care 2013;36:4036-42

24. Exalto LG, Whitmer RA, Kappele LJ, Biessels GJ. An update on type 2 diabetes, vascular dementia and Alzheimer's disease. Exp Gerontol 2012;47:858-64 
25. Almdal $\mathrm{T}$, Scharling $\mathrm{H}$, Jensen JS, Vestergaard $\mathrm{H}$. The independent effect of type 2 diabetes mellitus on ischemic heart disease, stroke, and death: a population-based study of 13,000 men and women with 20 years of follow-up. Arch Intern Med 2004;164:1422-6

26. Beckman JA, Creager MA, Libby P. Diabetes and atherosclerosis: epidemiology, pathophysiology, and management. JAMA 2002;287:2570-81

27. Biessels GJ, van der Heide LP, Kamal A, Bleys RL, Gispen WH. Ageing and diabetes: implications for brain function. Eur J Pharmacol 2002;441:1-14

28. Qiu WQ, Folstein MF. Insulin, insulin-degrading enzyme and amyloid-beta peptide in Alzheimer's disease: review and hypothesis. Neurobiol Aging 2006;27:190-8

29. Li J, Liu D, Sun L, Lu Y, Zhang Z. Advanced glycation end products and neurodegenerative diseases: mechanisms and perspective. J Neurol Sci 2012;317:1-5

30. Zieman SJ, Kass DA. Advanced glycation endproduct crosslinking in the cardiovascular system: potential therapeutic target for cardiovascular disease. Drugs 2004;64:459-70

31. Moran C, Munch G, Forbes JM, et al. Type 2 diabetes mellitus, skin autofluorescence and brain atrophy. Diabetes 2015;64:279-83

32. den Heijer T, Launer LJ, Prins ND, et al. Association between blood pressure, white matter lesions, and atrophy of the medial temporal lobe. Neurology 2005;64:263-7

33. Kohler S, Baars MA, Spauwen P, Schievink S, Verhey FR, van Boxtel MJ. Temporal evolution of cognitive changes in incident hypertension: prospective cohort study across the adult age span. Hypertension 2014;63:245-51

34. Prins ND, van Dijk EJ, den Heijer $T$, et al. Cerebral small-vessel disease and decline in information processing speed, executive function and memory. Brain 2005;128:2034-41

35. Creager MA, Luscher TF, Cosentino F, Beckman JA. Diabetes and vascular disease: pathophysiology, clinical consequences, and medical therapy: Part I. Circulation 2003;108:1527-32

36. Kim YS, Immink RV, Stok WJ, Karemaker JM, Secher NH, van Lieshout JJ. Dynamic cerebral autoregulatory capacity is affected early in Type 2 diabetes. Clin Sci (Lond) 2008;115:255-62

37. Low PA, Benrud-Larson LM, Sletten DM, et al. Autonomic symptoms and diabetic neuropathy: a population-based study. Diabetes Care 2004;27:2942-7

38. Khan U, Porteous L, Hassan A, Markus HS. Risk factor profile of cerebral small vessel disease and its subtypes. J Neurol Neurosurg Psychiatry 2007;78:702-6

39. Gaens KH, Stehouwer CD, Schalkwijk CG. Advanced glycation endproducts and its receptor for advanced glycation endproducts in obesity. Curr Opin Lipidol 2013;24:4-11

40. Aziz NA, van der Marck MA, Pijl H, Olde Rikkert MG, Bloem BR, Roos RA. Weight loss in neurodegenerative disorders. J Neurol 2008;255:1872-80

41. Berger AK, Fratiglioni L, Forsell Y, Winblad B, Backman L. The occurrence of depressive symptoms in the preclinical phase of AD: a population-based study. Neurology 1999;53:1998-2002

42. Mesholam RI, Moberg PJ, Mahr RN, Doty RL. Olfaction in neurodegenerative disease: a meta-analysis of olfactory functioning in Alzheimer's and Parkinson's diseases. Arch Neurol 1998;55:84-90

43. Holden KF, Lindquist K, Tylavsky FA, et al. Serum leptin level and cognition in the elderly: Findings from the Health ABC Study. Neurobiol Aging 2009;30:1483-9

44. Lieb W, Beiser AS, Vasan RS, et al. Association of plasma leptin levels with incident Alzheimer disease and MRI measures of brain aging. JAMA 2009;302:2565-72

45. Doherty TJ. Invited review: Aging and sarcopenia. J Appl Physiol (1985) 2003;95:1717-27

46. Mrak RE, Griffin WS. Interleukin-1, neuroinflammation, and Alzheimer's disease. Neurobiol Aging 2001;22:903-8

47. Reijmer YD, van den Berg E, Ruis C, Kappelle $L$, Biessels GJ. Cognitive dysfunction in patients with type 2 diabetes. Diabetes Metab Res Rev;26:507-19

48. Biessels GJ, Deary IJ, Ryan CM. Cognition and diabetes: a lifespan perspective. Lancet Neurol 2008;7:184-90

49. Yaffe K. Metabolic syndrome and cognitive decline. Curr Alzheimer Res 2007;4:123-6

50. Luchsinger JA, Reitz C, Honig LS, Tang MX, Shea S, Mayeux R. Aggregation of vascular risk factors and risk of incident Alzheimer disease. Neurology 2005;65:545-51

51. Yaffe K, Weston AL, Blackwell T, Krueger KA. The metabolic syndrome and development of cognitive impairment among older women. Arch Neurol 2009;66:324-8 
52. Deary IJ, Strand S, Smith P, Fernandes C. Intelligence and educational achievement. Intelligence 2007;35:13-21

53. Schillinger D, Barton LR, Karter AJ, Wang F, Adler N. Does literacy mediate the relationship between education and health outcomes? A study of a low-income population with diabetes. Public Health Rep 2006;121:245-54

54. Deary IJ, Spinath FM, Bates TC. Genetics of intelligence. Eur J Hum Genet 2006;14:690-700

55. Deary IJ, Johnson W. Intelligence and education: causal perceptions drive analytic processes and therefore conclusions. Int J Epidemiol 2010;39:1362-9

56. Yaffe K, Lindquist K, Schwartz AV, et al. Advanced glycation end product level, diabetes, and accelerated cognitive aging. Neurology 2011;77:1351-6

57. van den Berg E, Kloppenborg RP, Kessels RP, Kappelle LJ, Biessels GJ. Type 2 diabetes mellitus, hypertension, dyslipidemia and obesity: A systematic comparison of their impact on cognition. Biochim Biophys Acta 2009;1792:470-81

58. Nooyens AC, Baan CA, Spijkerman AM, Verschuren WM. Type 2 diabetes and cognitive decline in middle-aged men and women: the Doetinchem Cohort Study. Diabetes Care 2010;33:1964-9

59. Tuligenga RH, Dugravot A, Tabak AG, et al. Midlife type 2 diabetes and poor glycaemic control as risk factors for cognitive decline in early old age: a post-hoc analysis of the Whitehall II cohort study. Lancet Diabetes Endocrinol 2014;2:228-35

60. Yaffe K, Falvey C, Hamilton N, et al. Diabetes, glucose control, and 9-year cognitive decline among older adults without dementia. Arch Neurol 2012;69:1170-5

61. Qiu C, Winblad B, Fratiglioni L. The age-dependent relation of blood pressure to cognitive function and dementia. Lancet Neurol 2005;4:487-99

62. Reitz C, Luchsinger JA. Relation of Blood Pressure to Cognitive Impairment and Dementia. Curr Hypertens Rev 2007;3:166-76

63. Gomar JJ, Bobes-Bascaran MT, Conejero-Goldberg C, Davies P, Goldberg TE, Alzheimer's Disease Neuroimaging I. Utility of combinations of biomarkers, cognitive markers, and risk factors to predict conversion from mild cognitive impairment to Alzheimer disease in patients in the Alzheimer's disease neuroimaging initiative. Arch Gen Psychiatry 2011;68:961-9

64. Eckerstrom $C$, Olsson $E$, Bjerke $M$, et al. A combination of neuropsychological, neuroimaging, and cerebrospinal fluid markers predicts conversion from mild cognitive impairment to dementia. J Alzheimers Dis 2013;36:421-31

65. Jack CR, Jr., Knopman DS, Jagust WJ, et al. Hypothetical model of dynamic biomarkers of the Alzheimer's pathological cascade. Lancet Neurol 2010;9:119-28

66. Kodl CT, Seaquist ER. Cognitive dysfunction and diabetes mellitus. Endocr Rev 2008;29:494-511

67. Awad N, Gagnon M, Messier C. The relationship between impaired glucose tolerance, type 2 diabetes, and cognitive function. J Clin Exp Neuropsychol 2004;26:1044-80

68. Saczynski JS, Jonsdottir MK, Garcia ME, et al. Cognitive impairment: an increasingly important complication of type 2 diabetes: the age, gene/environment susceptibility--Reykjavik study. Am J Epidemiol 2008;168:1132-9

69. Palta P, Schneider AL, Biessels GJ, Touradji P, Hill-Briggs F. Magnitude of cognitive dysfunction in adults with type 2 diabetes: a meta-analysis of six cognitive domains and the most frequently reported neuropsychological tests within domains. J Int Neuropsychol Soc 2014;20:278-91

70. Jolles J, Houx PJ, van Boxtel MP, Ponds RW, (Eds.). Maastricht Aging Study: Determinants of cognitive aging. Maastricht, Neuropsych Publishers, 1995

71. Schram MT, Sep SJ, van der Kallen CJ, et al. The Maastricht Study: an extensive phenotyping study on determinants of type 2 diabetes, its complications and its comorbidities. Eur J Epidemiol 2014;29:43951

72. Harris TB, Launer L, Eiriksdottir G, et al. Age, Gene/Environment Susceptibility-Reykjavik Study: multidisciplinary applied phenomics. Am J Epidemiol 2007;165:1076-87

73. Alibhai SM, Greenwood C, Payette H. An approach to the management of unintentional weight loss in elderly people. CMAJ 2005;172:773-80

74. Kickbusch I, Pelikan JM, Apfel F, Tsouros A. Health Literacy: The Solid Facts. Copenhagen, World Health Organization, 2013

75. Qiu C, von Strauss E, Backman L, Winblad B, Fratiglioni L. Twenty-year changes in dementia occurrence suggest decreasing incidence in central Stockholm, Sweden. Neurology 2013;80:1888-94 
76. Schrijvers EM, Verhaaren BF, Koudstaal PJ, Hofman A, Ikram MA, Breteler MM. Is dementia incidence declining?: Trends in dementia incidence since 1990 in the Rotterdam Study. Neurology 2012;78:1456-63

77. Richard E, Moll van Charante EP, van Gool WA. Vascular risk factors as treatment target to prevent cognitive decline. J Alzheimers Dis 2012;32:733-40

78. Forette $\mathrm{F}$, Seux ML, Staessen JA, et al. The prevention of dementia with antihypertensive treatment: new evidence from the Systolic Hypertension in Europe (Syst-Eur) study. Arch Intern Med 2002;162:2046-52

79. Peters $\mathrm{R}$, Beckett $\mathrm{N}$, Forette $\mathrm{F}$, et al. Incident dementia and blood pressure lowering in the Hypertension in the Very Elderly Trial cognitive function assessment (HYVET-COG): a double-blind, placebo controlled trial. Lancet Neurol 2008;7:683-9

80. Deckers K, van Boxtel MP, Schiepers OJ, et al. Target risk factors for dementia prevention: a systematic review and Delphi consensus study on the evidence from observational studies. Int J Geriatr Psychiatry 2015; 30: 234-46 
SUMMARY 


\section{Summary}

The central topic of the studies in this thesis was to examine the relationship between type 2 diabetes and cognitive functioning. In the first chapters, the association of 1) type 2 diabetes, 2) its associated vascular risk factors and 3) body composition with cognitive functioning were examined. The last experimental chapter was focused on the associations of verbal intelligence with diabetic complications and walking speed in individuals with type 2 diabetes.

This summary provides an overview of the six studies described in the separate chapters of this thesis. In Chapter 2, the effect of baseline and incident type 2 diabetes on cognitive change over 12 years was examined. In this chapter individuals with type 2 diabetes at baseline showed a three times larger decline in information processing speed and a 4 times larger decline in executive function compared with those without diabetes. Effects on memory were less pronounced. Individuals who developed diabetes during the study showed signs of early decline in information processing speed compared with those who did not have diabetes during the study. Disease exposure seemed to play a role in the development of cognitive decline. Our results may provide a window of opportunity for prevention and early treatment of diabetes-related cognitive deficits.

In Chapter 3, we examined the association between skin autofluorescence (SAF), a non-invasive marker for tissue advanced glycation end-products (AGEs), and plasma AGEs with cognitive functions. We showed inverse associations of SAF with memory, which were attenuated and became non-significant after adjustment for vascular (risk) factors and depression. We found no robust associations of plasma AGEs with cognitive performance. Associations did not differ significantly between individuals with and without diabetes. Our results may suggest that AGEs are involved in the development of cognitive decline, particularly memory decline, and possibly in part through the effects of vascular (risk) factors.

In Chapter 4, linear and quadratic associations of ambulatory blood pressure with cognitive performance and associations of dipping status with cognitive performance were examined in individuals with and without type 2 diabetes. Results of this chapter showed significant quadratic (inverted U-shaped) associations of 24-hour diastolic blood pressure (DBP) with information processing speed and memory diabetes in individuals with type 2 , but not in those without. These associations could not be explained by potential confounders. Interactions between diabetes status and quadratic 24-hour DBP on these cognitive functions were also significant. No clear pattern could be found in the results for dipping status. These results suggest that not only high blood pressure levels, but also low blood pressure levels may increase the risk of cognitive impairment in individuals with type 2 diabetes, which may be 
important to consider in the therapeutic management of (diastolic) blood pressure levels in those individuals.

In Chapter 5, we investigated associations of visceral fat, abdominal and thigh subcutaneous fat, and thigh muscle with cognitive status in older individuals. Results showed that a higher amount of abdominal and thigh subcutaneous fat were associated with a decreased likelihood of dementia in women. These associations were attenuated by the degree of change in weight from midlife. Furthermore, a higher amount of muscle tissue was associated with a decreased likelihood of dementia in both men and women, independent of weight change. In addition, associations between body composition and dementia were strongest in women with a midlife $\mathrm{BMI}<25$. These findings suggest that it is important to monitor changes in weight when individuals, particularly women, enter old age.

In Chapter 6, we did not use cognitive performance as an outcome, but instead we used diabetic complications and walking speed as outcome measures and a cognitive variable, i.e. verbal intelligence, as a determinant. In this chapter we examined whether a lower verbal intelligence, as a core constituent of health literacy, was associated with the presence of diabetic complications and with walking speed, a measure of physical functioning and predictor of health status. Findings of this chapter showed that a lower verbal intelligence was associated with the presence of neuropathic pain and cardiovascular disease in individuals with type 2 diabetes. In addition, a lower verbal intelligence was associated with a slower walking speed. Interestingly, these associations could largely be explained by educational level. In order to reduce the risk of diabetic complications, it may therefore be important for clinicians to be well aware of the educational level of their patients and to tailor the information to a level that can be understood.

In the final chapter of this thesis, Chapter 7, all results that were presented in the previous 5 chapters were placed in a broader context, together with some reflection on methodological issues and the clinical and scientific implications of our studies. In addition, this last chapter includes a summary of the findings, a description of knowledge valorisation, a list of publications, acknowledgements, and a curriculum vitae. 
NEDERLANDSE SAMENVATTING 


\section{Nederlandse samenvatting}

Dementie is een syndroom dat gekenmerkt wordt door problemen met het denkvermogen, met name het geheugen, gedrag en de vaardigheid om alledaagse activiteiten uit te voeren. Helaas is het (nog) niet mogelijk om de ontwikkeling van dementie voor een lange tijd uit te stellen of te voorkomen. Daarom is het belangrijk om te onderzoeken welke risicofactoren aangepakt kunnen worden zodat de ontwikkeling van dementie vertraagd of zelfs voorkomen kan voorkomen. Belangrijke risicofactoren die samen lijken te hangen met dementie en die behandeld kunnen worden, zijn vasculaire risicofactoren zoals type 2 diabetes (ouderdomssuiker) en hoge bloeddruk. Om beter te begrijpen hoe deze factoren kunnen leiden tot dementie, is het belangrijk om te onderzoeken in welke mate vasculaire risicofactoren, zoals type 2 diabetes en hoge bloeddruk, samenhangen met een verminderde cognitieve prestatie, dat een voorspeller kan zijn voor de ontwikkeling van een cognitieve stoornis in een latere fase.

Het thema dat daarom centraal staat in dit proefschrift is de relatie tussen type 2 diabetes en cognitieve functies. We hebben gekeken of diabetes, versuikerde eiwitten, bloeddruk en lichaamssamenstelling samenhangen met cognitieve functies. Daarnaast hebben we de relatie tussen verbale intelligentie en diabetes complicaties en tussen verbale intelligentie en loopsnelheid onderzocht in mensen met diabetes.

In Hoofdstuk 2 hebben we de effecten van type 2 diabetes op cognitieve verandering over een periode van 12 jaar onderzocht. De resultaten toonden aan dat mensen met diabetes 3 keer zo snel achteruitgingen op een taak voor informatieverwerkingssnelheid en 4 keer zo snel op een taak voor uitvoerende controlefuncties in vergelijking met mensen die geen diabetes hadden. De effecten op het geheugen waren minder sterk. Mensen die gedurende de onderzoeksperiode diabetes ontwikkelden, lieten een subtiele achteruitgang in informatieverwerkingssnelheid zien vergeleken met mensen die geen diabetes hadden gedurende de onderzoeksperiode. Ziekteduur bleek een rol te spelen in de ontwikkeling van cognitieve achteruitgang. Deze onderzoeksresultaten geven aan dat diabetes een risicofactor is voor cognitieve achteruitgang. Daarnaast geven ze aan dat het voorkomen van diabetes of een vroegtijdige behandeling van diabetes mogelijk de kans op de ontwikkeling van een cognitieve stoornis verkleint.

In Hoofdstuk 3 hebben we gekeken naar versuikerde eiwitten, die een rol lijken te spelen bij de ontwikkeling van dementie. Deze eiwitten werden gemeten in het weefsel (in de huid) en in het bloed. De resultaten toonden aan dat het hebben van meer versuikerde eiwitten in de huid samenhangt met een slechter geheugen en dat vasculaire (risico)factoren hierin een rol lijken te spelen. We vonden geen relaties tussen versuikerde eiwitten in het bloed en cognitieve prestatie. Onze resultaten 
zouden erop kunnen wijzen dat versuikerde eiwitten in het weefsel een rol spelen in de ontwikkeling van cognitieve achteruitgang, vooral achteruitgang in geheugen.

In Hoofdstuk 4 werden relaties tussen bloeddruk, gemeten over 24 uur, en cognitieve functies onderzocht. De resultaten toonden aan dat zowel een lage als een hoge diastolische bloeddruk (onderdruk) samenhangt met een lagere prestatie op taken voor informatieverwerkingssnelheid en geheugen. We vonden deze relatie bij mensen met diabetes, maar niet bij mensen zonder diabetes. Deze resultaten lijken aan te geven dat niet alleen een hoge bloeddruk, maar ook een lage bloeddruk het risico op het ontwikkelen van cognitieve problemen kan verhogen in mensen met diabetes. Het is daarom belangrijk om zowel de bloeddruk als het cognitief functioneren van mensen met diabetes regelmatig te meten en in de gaten te houden. We hebben in dit hoofdstuk ook gekeken naar de relatie tussen het verschil in dag- en nachtbloeddruk ('dipping status') en cognitieve functies, maar we konden geen eenduidige relaties vinden.

In Hoofdstuk 5 hebben we onderzocht of de hoeveelheid vet en de hoeveelheid spierweefsel samenhangen met cognitieve status (milde cognitieve stoornis of dementie) in ouderen. Een grotere hoeveelheid onderhuids vet (in de buik en in het dijbeen) hing samen met een kleinere kans op dementie bij oudere vrouwen. Deze relaties werden minder sterk wanneer er rekening gehouden werd met gewichtsverandering tussen middelbare en oudere leeftijd. Daarnaast hing een grotere hoeveelheid spierweefsel in het dijbeen samen met een kleinere kans op dementie bij zowel mannen als vrouwen. Gewichtsverandering bleek hier geen (grote) rol in te spelen. De resultaten suggereren dat het belangrijk kan zijn om veranderingen in gewicht in de gaten te houden wanneer mensen, vooral vrouwen, ouder worden.

In Hoofdstuk 6 hebben we gekeken naar de relaties tussen aan de ene kant verbale intelligentie en aan de andere kant diabetescomplicaties en loopsnelheid. Diabetes is een complexe ziekte en om diabetescomplicaties, zoals hart- en vaatziekten, te voorkomen is het belangrijk dat diabetespatiënten goed op hun gezondheid letten door bijvoorbeeld gezond te eten en medicatie te gebruiken. Daarvoor hebben zij vaardigheden nodig om gezondheidsinformatie goed te kunnen begrijpen en te gebruiken. Deze vaardigheden worden ook wel gezondheidsgeletterdheid ('health literacy') genoemd. Verbale intelligentie kan beschouwd worden als een belangrijk onderdeel van gezondheidsgeletterdheid en zou daarom kunnen samenhangen met een groter risico op het krijgen van diabetescomplicaties. Onze resultaten lieten zien dat een lagere verbale intelligentie inderdaad samenhangt met de aanwezigheid van neuropathische pijn (zenuwpijn) en hart- en vaatziekten in mensen met diabetes. Daarnaast was een lagere verbale intelligentie geassocieerd met een lagere loopsnelheid. Deze relaties konden voor een groot deel verklaard worden door opleidingsniveau. Het zou daarom belangrijk kunnen zijn dat artsen zich 
bewust zijn van het opleidingsniveau van hun patiënten en dat zij de manier waarop ze informatie verschaffen, aanpassen aan het niveau van de patiënt. Dit zou er uiteindelijk voor kunnen zorgen dat de kans om complicaties te ontwikkelen kleiner wordt voor diabetespatiënten.

In het laatste hoofdstuk, Hoofdstuk 7, zijn alle resultaten van dit proefschrift in een bredere context geplaatst en hebben we de onderzoeksmethodes bediscussieerd. Daarnaast hebben we besproken wat onze resultaten kunnen betekenen voor de kliniek en de wetenschap. De laatste onderdelen van dit hoofdstuk zijn: een samenvatting van de resultaten, een lijst van publicaties, een valorisatieparagraaf, het dankwoord, en het curriculum vitae van de auteur. 
PUBLICATIONS 


\section{Publications}

\section{Thesis}

Spauwen PJ, Köhler S, Verhey FR, Stehouwer CD, van Boxtel MP. Effects of type 2 diabetes on 12-year cognitive change: results from the Maastricht Aging Study. Diabetes Care 2013;36:1554-61

Spauwen PJ, Stehouwer CD. Cognitive decline in type 2 diabetes. Lancet Diabetes Endocrinol 2014;2:188-9

Spauwen PJ, van Eupen MG, Kohler S, Stehouwer CD, Verhey FR, van der Kallen CJ, Sep SJ, Koster A, Schaper NC, Dagnelie PC, Schalkwijk CG, Schram MT, van Boxtel MP. Associations of advanced glycation end-products with cognitive functions in individuals with and without type 2 diabetes: the Maastricht Study. J Clin Endocrinol Metab 2015;100:951-60

Spauwen PJ, van Boxtel MP, Verhey FR, Kohler S, Sep SJ, Koster A, Dagnelie PC, Henry RM, Schaper NC, van der Kallen CJ, Schram MT, Kroon AA, Stehouwer CD. Both low and high 24-hour diastolic blood pressure are associated with worse cognitive performance in type 2 diabetes: The Maastricht Study. Diabetes Care 2015;38:1473-80

Spauwen PJ, Murphy RA, Jonsson PV, Sigurdsson S, Garcia ME, Eiriksdottir G, van Boxtel MP, Lopez OL, Gudnason V, Harris TB, Launer LJ. Associations of fat and muscle tissue with cognitive status in older adults: The AGES-Reykjavik Study. Submitted

Spauwen PJ, Martens RJ, Stehouwer CD, Verhey FR, Schram MT, Sep SJ, van der Kallen CJ, Dagnelie PC, Henry RM, Schaper NC, van Boxtel MP. Lower verbal intelligence is associated with diabetic complications and slower walking speed in individuals with type 2 diabetes: The Maastricht Study. Submitted

\section{Other}

Kohler S, Baars MA, Spauwen PJ, Schievink S, Verhey FR, van Boxtel MJ. Temporal evolution of cognitive changes in incident hypertension: prospective cohort study across the adult age span. Hypertension 2014;63:245-51 
KNOWLEDGE VALORISATION 


\section{Knowledge valorisation}

Knowledge valorisation refers to the "process of creating value from knowledge, by making knowledge suitable and/or available for social (and/or economic) use, and by making knowledge suitable for translation into competitive products, services, processes and new commercial activities" (adapted definition based on the National Valorisation Committee 2011:8).

Mr. D. is a 70-year-old man who lives together with his wife. His wife has noticed that her husband has some problems with his memory. She does not know whether these memory problems are normal for his age. She is afraid her husband is developing dementia and decides to make an appointment with their general practitioner. The general practitioner concludes that Mr. D. should be referred to a neuropsychologist and has seen in Mr.D's medical record that he is diagnosed with diabetes and hypertension for a few years. Could there be a relation between his blood pressure and diabetes and his cognitive problems? Is there a possibility for Mr.D. to prevent further cognitive decline? How could the neuropsychologist and the internist play a role in this? And how could Mr. D and his wife benefit from results of this thesis?

\section{Societal relevance}

What is the social (and/or economic) relevance of the research results (i.e. in addition to the scientific relevance)?

Nowadays almost every person in the world knows someone who suffers from dementia. Dementia is a progressive disease that is characterized by decline in mental abilities, also known as cognitive functions. Specifically, individuals with dementia suffer from decline in memory function. Decline in cognitive functions is, however, not the only symptom of dementia as patients with dementia can also suffer from behavioral problems and depression. This can lead to a large decline in quality of life and to a huge burden for caregivers, i.e. spouses or children of the patient.

Dementia is highly prevalent worldwide; In 2010 it was estimated that 35.6 million people lived with dementia and these numbers are expected to almost double every 20 years to 65.7 million in 2030 and 115.4 million in 2050 . In addition, the worldwide societal economic was estimated at US \$ 604 billion in 2010. These alarming numbers indicate that it is very important to find a treatment to delay or prevent the development of dementia.

However, there is still no effective treatment to significantly delay the onset of dementia. Therefore, it is important to identify risk factors for dementia that can be treated. An average five-year delay in the age of onset of dementia would tend to reduce population prevalence by $50 \%$ and thereby greatly reduce the impact of 
dementia in the general population. Furthermore, recent studies have suggested that the incidence of dementia is decreasing, possibly as a result of improved control of vascular risk factors (e.g. hypertension) and cardiovascular disease. Therefore, studies are needed that examine how these risk factors can be modified and whether better control of these risk factors could indeed at least significantly delay the development of dementia.

Dementia is preceded by a phase of cognitive decline in which individuals show memory problems, but are not demented yet. This phase is particularly important, because in this phase further memory decline may be prevented. It would therefore be important to focus on risk factors for cognitive decline that may precede dementia diagnosis.

In this thesis we have identified certain modifiable risk factors, i.e. diabetes, low and high blood pressure, and high accumulation of certain proteins in tissue, called advanced glycation end-products (AGEs). This thesis stresses that individuals in whom these risk factors are present may have an increased risk of developing cognitive problems. Since adopting a healthy lifestyle can ameliorate the impact of these risk factors and can even prevent their development, it is very important that people are aware of the negative effects of these risk factors on the brain. If people start to adopt a healthy lifestyle, the prevalence of dementia may decrease dramatically.

In addition, physicians should become aware of these risk factors and should see them as "red flags" for cognitive decline. Physicians should monitor both blood pressure and blood sugar levels, and maybe in the future also the accumulation of AGEs in skin tissue, together with cognitive functioning in order to potentially delay or prevent the development of cognitive decline and dementia.

\section{Target groups}

To whom, in addition to the academic community, are your research results of interest and why?

Results of this thesis are important for all individuals. They implicate that prevention or control of diabetes and high (hyper) or low (hypo) blood pressure may prevent or delay cognitive decline. Ideally, this prevention or control should start early on in life by adopting a healthy lifestyle. Therefore, people should become aware of their risk of developing cognitive problems. Our results are particularly important for individuals who are middle-aged and have diabetes and hyper- or hypotension. These individuals should check their blood sugar levels and blood pressure and should visit their physicians regularly. As mentioned previously, physicians should follow-up their patients in whom these risk factors are present. Another target group is the pharmaceutical industry. Results of this thesis should encourage drug industries or diabetes specialists to invent new treatment or adjust their current treatments for diabetes and hypertension. New or intensified treatments that both control sugar or 
blood pressure levels and prevent or delay cognitive decline would be like killing two birds with one stone. It would be particularly interesting for pharmaceutical industries to develop treatments to reduce accumulation of AGEs. This thesis shows that AGEs are related to cognitive functions and may therefore be a new target for treatment. AGEs have also been associated with dementia and with vascular disease and could therefore be a potential new promising candidate for the prevention of or delaying the development of dementia. However, more research into the effect of AGE accumulation on dementia is warranted, because this is a relatively new research area.

Not only treatment of diabetes and other vascular risk factors could potentially affect cognitive functioning, also extra education for patients in whom these risk factors are present, especially for those who may have difficulties with understanding the impact of their disease, may help to manage diabetes, blood pressure and cognitive functions. Diabetes nurses could provide this education, and these educational programs may be developed by universities or psychologists who are specialists in providing educational training.

\section{Innovation/Products}

To what degree can your results be called innovative in respect to the existing range of products, services, and processes?

The most innovative part of this thesis involves the measurement of skin autofluorescence (SAF) with a SAF reader. This reader measures fluorescence of certain AGEs (proteins that are bound to sugar) in the skin (volar side of the forearm) and this is assumed to reflect not only accumulation of AGEs in the arm, but in other parts of the body, like the brain, as well. We have shown, that this fluorescence, as a marker of tissue AGE accumulation, is associated with cognitive functions, particularly memory. The SAF reader may therefore become a tool for the risk assessment of cognitive impairment and dementia in clinical settings. However, more research is needed to examine the predictive value of this tool, especially in patient groups. Our research should be a regarded as a promising starting point for future studies, which may lead to the development of a new biomarker and non-invasive tool for the risk assessment of dementia.

\section{Implementation}

How can the results of this thesis contribute to implementation of new protocols and assessments in the clinical settings?

The clinical importance of this thesis involves the identification of certain vascular risk factors, especially type 2 diabetes, for cognitive decline. It is important for clinicians to get insight into the vascular risk profile, including diabetes status and 
blood pressure, and to monitor these factors as well as cognitive performance. Better control of diabetes and blood pressure may contribute to less change in cognitive functions over time. Therefore, cognitive functioning should be included in the checklist of physicians who are specialized in metabolic disease (e.g. internists). In addition, information about vascular risk factors (blood pressure and diabetes status) should be gathered during the anamneses by a neuropsychologist/psychiatrist. This could help to identify individuals who are at increased risk for cognitive impairment and could make early intervention and treatment possible.

The assessment of AGE accumulation could also become an important point on the checklist of an internist and psychologist/psychiatrist. However, more research is needed into the predictive value of skin autofluorescence for dementia. Therefore, the SAF reader should first be used for research at memory clinics to examine whether patients with a diagnosis of Mild Cognitive Impairment (individuals with cognitive complaints which are not (yet) severe enough to be regarded as dementia) or dementia show higher SAF levels than individuals without a clinical diagnosis of cognitive impairment (those with subjective cognitive complaints).

Furthermore, larger studies should examine whether SAF can predict who will develop cognitive impairments and who will not. Therefore studies that follow up individuals for a certain amount of time (several years) are needed. If the SAF values indeed appear to predict the development of cognitive impairment, the SAF reader can be implemented in clinical settings, which will be feasible since the measurement is non-invasive and takes only three minutes to complete. The reader can then be used in addition to neuropsychological assessments, brain scans, and other biomarkers for the prediction of the development of dementia.

The ultimate goal of studying modifiable risk factors for cognitive decline and dementia will be to delay or prevent the development of dementia. Several large randomized control trials (RCT) have examined the effect of treatment of vascular risk factors on dementia and cognitive decline. Most of these studies have focused on treatment of one single vascular risk factor and the treatment of hypertension seemed to have the most promising effect. However, most studies failed to find an effect on dementia and cognitive decline, which may be due to the study design. For example, follow-up durations may have been too short and the age of the sample may have been too high. The Innovative, Midlife intervention for Dementia Deterrence (InMINDD) is a new study that will develop and validate online tools to help physicians to assess if an individual's lifestyle supports long-term brain health. This study will generate personal strategies for adopting a brain healthy lifestyle by stimulating change in risk factors for dementia, including smoking, blood pressure, and cholesterol. Studies, like In-MINDD, hopefully increase our understanding about how to delay or prevent dementia. One of the main advantages of In-MINDD is the multifactorial approach, which is supported by the results of this thesis. These suggest that multiple vascular risk factors can increase the risk for cognitive impairment and 
provide a starting point for new studies and provide relevant information for clinicians who should regard cognitive decline and dementia as multifactorial disorders. These multiple factors, which not only include diabetes and hypertension, but also, for example, depression, smoking, and cholesterol levels, should therefore all be considered when trying to increase brain health.

\section{So, how could Mr. D. and his wife benefit from the results of this thesis?}

If the recommendations of this thesis are followed, the neuropsychologist would know that Mr.D has been diagnosed with diabetes and hypertension. After a neuropsychological assessment, a skin autofluorescence measurement could be performed for research goals so hopefully in the future this assessment can contribute to dementia risk assessment. The neuropsychologist informs the internist about Mr. D's cognitive problems and tells Mr. D. that it is very important to adopt a healthy lifestyle, because this may contribute to a healthier brain. Mr. D. is referred to his internist which from now on will not only monitor his blood sugar levels and blood pressure, but will also pay attention to his cognition in cooperation with the neuropsychologist. Mr. D. will try to increase his brain health by adopting a healthier lifestyle, because he wants to do everything he can to increase his brain health. 
ACKNOWLEDGEMENTS/DANKWOORD 


\section{Acknowledgements/dankwoord}

Er staat 1 auteur op de voorkant van dit proefschrift, maar een proefschrift kan natuurlijk niet tot stand komen zonder de hulp van andere mensen. Ik wil dan ook graag iedereen bedanken die mij geholpen heeft met de totstandkoming van dit proefschrift.

Als eerste wil ik mijn promotieteam bedanken voor de steun en expertise.

\section{Mijn promotoren}

Beste professor Verhey, beste Frans, allereerst bedankt dat ik 4,5 jaar op deze afdeling heb mogen werken en mezelf heb kunnen ontwikkelen als wetenschappelijk onderzoeker. Ook bedankt voor je interesse in mijn werk en de feedback die ik gekregen heb om mijn artikels te verbeteren.

Beste professor Stehouwer, beste Coen, bedankt voor alle feedback en suggesties die ik gekregen heb. Ik heb enorm veel van je geleerd en heb veel bewondering voor de hoeveelheid kennis die jij beschikt. Ik kon altijd op je rekenen.

\section{Mijn co-promotor}

Beste Martin, bedankt voor je bijdrage aan mijn ontwikkeling als onderzoeker en voor alle hulp die je me gegeven hebt. Ik wil je vooral bedanken voor je rust en geduld. Dankzij jou kwam mijn scherpe blik weer terug als ik het even niet meer zag zitten en kon ik mijn rust terugvinden. Ontzettend bedankt dat je mijn begeleider was en vooral voor de manier waarop.

De leden van de beoordelingscommissie

Ik wil graag de leden van de beoordelingscommissie, prof. dr. R. Ponds, prof. dr. G.J. Biessels, prof. dr. H. Bosma, prof. dr. C. Gaillard en dr. M. Van Greevenbroek bedanken voor het nemen van de tijd om mijn proefschrift te lezen en te beoordelen.

\section{All collaborators abroad}

Dear Dr. Launer, thank you for giving me the oppurtunity to work abroad at the NIA. I learned a lot from you and hopefully we'll meet again in the future. I also want to thank my other collegues at the NIA (Tamara, Tad, Melissa, Julia, Caroline, Robert, Ming, Jennifer, Rachel, Michael, Blossom, and Ilse) for the cooperation, the kindness, and for hanging out with me and showing me around Bethesda and Washington D.C.

\section{Alle co-auteurs}

Beste co-auteurs, waaronder ook veel leden van het managementteam van De Maastricht Studie, bedankt voor jullie kritische blik en feedback op mijn artikels. Een speciaal woord van dank aan Sebastian Köhler. Dankzij jou werd het niveau van de statistische analyses een stuk hoger en daarmee ook de kwaliteit van de artikels. 
Bedankt dat je altijd tijd voor me had en dat je me zo goed geholpen hebt! Ik heb veel van je geleerd!

I would like to thank the co-authors from the AGES-Reykjavik Study, dr. S. Sigurdsson, dr. P.V. Jonsson, dr. V. Gudnason, dr. O.L. Lopez, and G. Eiriksdottir for their inputs and collaboration.

Alle medewerkers van De Maastricht Studie

Tijdens mijn promotietraject heb ik op twee locaties gewerkt; in het onderzoekscentrum van De Maastricht Studie en bij de afdeling Psychiatrie en Neuropsychologie.

Ten eerste wil ik alle medewerkers van De Maastricht Studie bedanken. Bedankt voor jullie inzet, maar ook voor jullie gezelligheid. Ondanks dat het soms zware werkdagen waren, kon ik altijd rekenen op jullie aangename gezelschap. Verder wil ik de deelnemers van De Maastricht Studie bedanken voor hun deelname. Zonder jullie had ik deze mooie data niet kunnen gebruiken.

Vooral ook met de promovendi van De Maastricht Studie heb ik een leuke tijd beleefd. Marcelle en Remy, bedankt voor de fijne samenwerking en voor jullie hulp bij het schrijven van hoofdstuk 3 en 6 . Ik vind het ontzettend leuk dat wij samen op een artikel staan! Fleur, jij was mijn cognitie-buddy. We hebben samen veel geklaagd, maar ook veel plezier beleefd. We sleepten elkaar erdoorheen als we weer eens elkaars vakantiedagen moesten opvangen. Frank, Stefan, Thomas, Julianne (bedankt voor de laatste taalkundige check van dit proefschrift!), Jose, Dennis, Pauline, Louise, Eline, Marja, Annemariek, Jeroen en Ben, met jullie heb ik ook nog (veel) samengewerkt bij De Maastricht Studie. Ook na werktijd zijn we nog regelmatig leuke dingen gaan doen. Bedankt allemaal voor de leuke tijd!

Alle medewerkers van de afdeling Psychiatrie en Neuropsychologie

Ik had het geluk dat er op mijn andere werklocatie ook fijne collega's werkten waarmee ik een leuke tijd beleefd heb. Ik wil alle promovendi en andere medewerkers van de afdeling Psychiatrie en Neuropsychologie bedanken voor de fijne tijd en de hulp die ik kreeg als ik hierom vroeg. Een speciaal woord van dank aan Nico Roozendaal. Jij stond altijd klaar als ik een "ICT-gerelateerd" vraagje had. Bedankt dat je het leven van vele medewerkers op onze afdeling een stukje makkelijker maakt. Ook Astrid wil ik bedanken voor haar hulp bij het inwerken van de cognitie medewerkers en voor het beantwoorden van mijn (vele) vragen.

De paranimfen en kamergenoten

De meeste steun op het werk heb ik gekregen van mijn lieve kamergenootjes en paranimfen, leke en Sanne. Jullie zijn de beste kamergenoten die iemand zich maar kan wensen. Bedankt voor jullie geduld, begrip en hulp. Ondanks dat jullie heel ander onderzoek doen, dachten jullie altijd met me mee en heb ik veel gehad aan jullie tips. 
Wie had gedacht dat ik ooit nog eens hetzelfde onderzoek als jullie ging doen! Vooral ook bedankt voor de gezelligheid op de kamer. We konden elkaar alles zeggen en hebben zeer prettig met elkaar samengewerkt. Ik kan me dan ook geen betere paranimfen wensen.

Mijn vrienden en familie

Mijn lieve vrienden en familie, bedankt voor jullie steun en interesse in mijn onderzoek. Maureen, Suzanne, Jasmijn, Stephanie, Maud, Marianne, Wendy (tevens bedankt voor het maken van de mooie kaft van dit proefschrift!), Sanne, Roy en Jim, bedankt voor jullie luisterende oor, jullie adviezen en vooral ook voor alle leuke dingen die we samen gedaan hebben. Ontspanning naast het werk is heel belangrijk en mede dankzij jullie was dit mogelijk!

Lieve pap en mam, bedankt voor jullie onvoorwaardelijke steun. Jullie kennen mij door en door en wisten dat het altijd goed zou komen als ik weer eens stress had. Jullie geloven voor $100 \%$ in mij en dat heeft mij altijd geholpen. Zonder jullie was ik niet zover gekomen als ik nu ben. Bedankt!

Molly, "woefke" van me, bedankt dat ik altijd op je knuffels kan rekenen en dat je na 16 jaar nog steeds ontzettend enthousiast bent als ik binnen kom lopen. Ik hoop dat ik nog wat jaartjes van je aanwezigheid mag genieten.

Lieve Stan, mijn grote vriendelijke reus, jij kwam in mijn leven toen ik bezig was met het afronden van mijn proefschrift. Zeker niet de makkelijkste periode om iemand te leren kennen. Maar wat heb jij een geduld. Bedankt voor je steun, voor het meedenken en je begrip als ik weer eens door de bomen het bos niet meer zag. Bedankt voor je eeuwige optimisme en vrolijkheid. Wat ben jij een goed mens! Ik ben ontzettend dankbaar dat ik jou in mijn leven heb. 
CURRICULUM VITAE 


\section{Curriculum Vitae}

Peggy Spauwen was born in Heerlen on December 31985 and grew up in Eijsden. After graduating from high school (Sint-Maartenscollege Maastricht) in 2004, she studied Psychology at Maastricht University. In 2007, Peggy started the Research Master Neuropsychology for which she performed a clinical internship and a research internship at the department of Medical Psychology of the Academic Hospital of Maastricht. In 2009, she graduated cum laude and started working as a research assistant on the project 'Brein en Leren' at Maastricht University. In 2010, Peggy worked a few weeks as a volunteer at a Boy's Home in India after which she started working as a PhD candidate at the Department of Psychiatry and Neuropsychology under supervision of Prof. dr. Frans Verhey, prof. dr. Coen Stehouwer, and Dr. Martin van Boxtel. As part of her PhD project, she worked at The Maastricht Study where she collected data for her project. During the last year of her PhD project, Peggy worked for 3 months at the National Institute on Aging in Bethesda (USA) to collaborate with Dr. Lenore Launer and colleagues. Currently, Peggy is working as a postdoctoral researcher at Maastricht University and as a neuropsychologist/postdoctoral researcher at Huize Padua (GGZ Oost-Brabant).

Peggy Spauwen is geboren in Heerlen op 3 december 1985 en groeide op in Eijsden. $\mathrm{Na}$ het afronden van het gymnasium aan het Sint-Maartenscollege te Maastricht, volgde ze de opleiding Psychologie aan de Universiteit Maastricht. In 2007 begon ze aan de onderzoeksmaster Neuropsychologie en als onderdeel van deze master heeft ze stage gelopen op de afdeling Medische Psychologie van het Academisch ziekenhuis in Maastricht. In 2009 studeerde ze cum laude af en is ze gaan werken als onderzoeksassistent voor het project 'Brein en Leren' aan de Universiteit Maastricht. In 2010 heeft Peggy een aantal weken vrijwilligerswerk gedaan in een opvanghuis voor jongens in India. Hierna is ze gaan werken als promovendus op de afdeling Psychiatrie en Neuropsychologie van de Universiteit Maastricht onder leiding van Prof. dr. Frans Verhey, Prof. dr. Coen Stehouwer en Dr. Martin van Boxtel. Peggy heeft tijdens haar promotietraject gewerkt bij De Maastricht Studie waar ze data verzamelde voor haar onderzoek. Daarnaast heeft ze in het laatste jaar van haar promotietraject 3 maanden bij the National Institute on Aging in Bethesda (VS) gewerkt om samen te werken met Dr. Lenore Launer en haar collega's. Momenteel werkt Peggy als postdoc onderzoeker bij de Universiteit Maastricht en als neuropsycholoog/senior onderzoeker bij Huize Padua (GGZ Oost Brabant). 
